\title{
POLYNOMIAL CHAOS AND SCALING LIMITS OF DISORDERED SYSTEMS
}

\author{
FRANCESCO CARAVENNA, RONGFENG SUN, AND NIKOS ZYGOURAS
}

\begin{abstract}
Inspired by recent work of Alberts, Khanin and Quastel AKQ14a, we formulate general conditions ensuring that a sequence of multi-linear polynomials of independent random variables (called polynomial chaos expansions) converges to a limiting random variable, given by a Wiener chaos expansion over the $d$-dimensional white noise. A key ingredient in our approach is a Lindeberg principle for polynomial chaos expansions, which extends earlier work of Mossel, O'Donnell and Oleszkiewicz MOO10. These results provide a unified framework to study the continuum and weak disorder scaling limits of statistical mechanics systems that are disorder relevant, including the disordered pinning model, the (long-range) directed polymer model in dimension $1+1$, and the two-dimensional random field Ising model. This gives a new perspective in the study of disorder relevance, and leads to interesting new continuum models that warrant further studies.
\end{abstract}

\section{Contents}

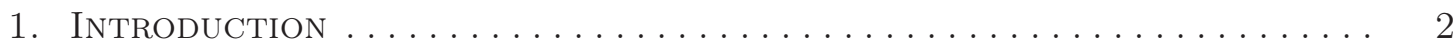

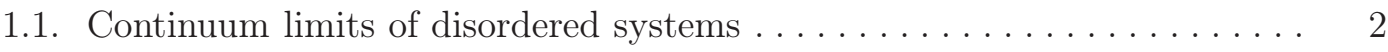

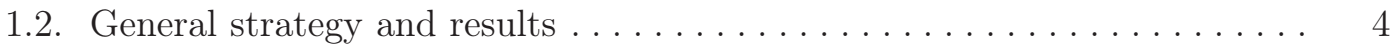

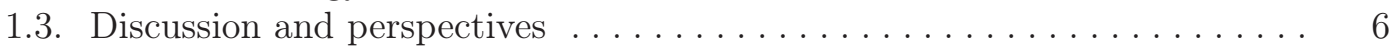

2. From polynomial to Wiener chaos via Lindeberg ............. 10

2.1. White noise in a nutshell . . . . . . . . . . . . . . . . . . 10

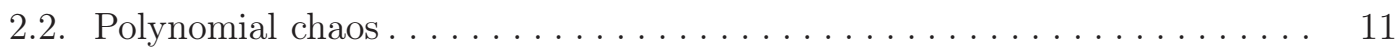

2.3. Convergence of polynomial chaos to Wiener chaos . . . . . . . . . . 11

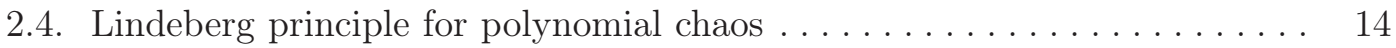

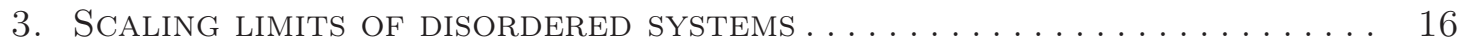

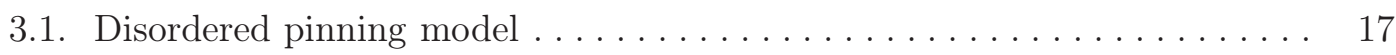

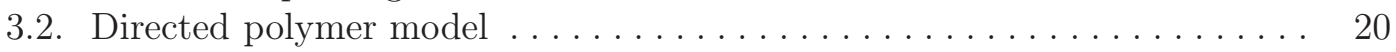

3.3. Random field Ising model . . . . . . . . . . . . . . . . . . . 24

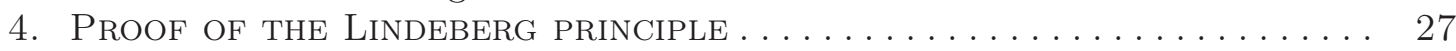

5. Proof of the Convergence to Wiener chaos $\ldots \ldots \ldots \ldots \ldots \ldots \ldots . \ldots \ldots$

6. PROOF FOR DISORDERED PINNING MODEL $\ldots \ldots \ldots \ldots \ldots \ldots \ldots \ldots \ldots \ldots$

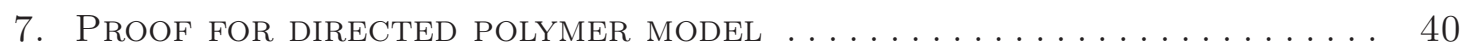

8. PROOF FOR RANDOM FIELD ISING MODEL $\ldots \ldots \ldots \ldots \ldots \ldots \ldots \ldots \ldots \ldots$

Appendix A. The Cameron-Martin Shift . . . . . . . . . . . . . . 49

Appendix B. Technical lemmas . . . . . . . . . . . . . . . . . 49

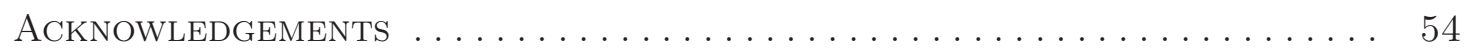

Date: October 26, 2016.

2010 Mathematics Subject Classification. Primary: 82B44; Secondary: 82D60, 60K35.

Key words and phrases. Continuum Limit, Finite Size Scaling, Lindeberg Principle, Polynomial Chaos, Wiener Chaos, Disordered Pinning Model, Directed Polymer Model, Random Field Ising Model. 


\section{INTRODUCTION}

In this paper, we consider statistical mechanics models defined on a lattice, in which disorder acts as an external "random field". We focus on models that are disorder relevant, in the sense that arbitrarily weak disorder changes the qualitative properties of the model. We will show that, when the homogeneous model (without disorder) has a non-trivial continuum limit, disorder relevance manifests itself via the convergence of the disordered model to a disordered continuum limit, if the disorder strength and lattice mesh are suitably rescaled.

Our approach is inspired by recent work of Alberts, Khanin and Quastel [AKQ14a] on the directed polymer model in dimension $1+1$. Here we follow a different path, establishing a general convergence result for polynomial chaos expansions based on a Lindeberg principle. This extends earlier work of Mossel, O'Donnell and Oleszkiewicz [MOO10] to optimal (second) moment assumptions, and is of independent interest.

In this section, we present somewhat informally the main ideas of our approach in a unified framework, emphasizing the natural heuristic considerations. The precise formulation of our results is given in Sections 2 and 3, which can be read independently (both of each other and of the present one). The proofs are contained in Sections 4 to 8 , while some technical parts have been deferred to the Appendixes. Throughout the paper, we use the conventions $\mathbb{N}:=\{1,2,3, \ldots\}$ and $\mathbb{N}_{0}:=\mathbb{N} \cup\{0\}$, and we denote by Leb the Lebesgue measure on $\mathbb{R}^{d}$.

1.1. ContinuUm limits of Disordered Systems. Consider an open set $\Omega \subseteq \mathbb{R}^{d}$ and define the lattice $\Omega_{\delta}:=(\delta \mathbb{Z})^{d} \cap \Omega$, for $\delta>0$. Suppose that a reference probability measure $\mathrm{P}_{\Omega_{\delta}}^{\mathrm{ref}}$ is given on $\mathbb{R}^{\Omega_{\delta}}$, which describes a real-valued field $\sigma=\left(\sigma_{x}\right)_{x \in \Omega_{\delta}}$. We focus on the case when each $\sigma_{x}$ takes two possible values (typically $\sigma_{x} \in\{0,1\}$ or $\sigma_{x} \in\{-1,1\}$ ).

Let $\omega:=\left(\omega_{x}\right)_{x \in \Omega_{\delta}}$ be i.i.d. random variables (also independent of $\sigma$ ) with zero mean, unit variance, and locally finite exponential moments, which represent the disorder. Probability and expectation for $\omega$ will be denoted respectively by $\mathbb{P}$ and $\mathbb{E}$.

Given $\lambda>0, h \in \mathbb{R}$ and a $\mathbb{P}$-typical realization of the disorder $\omega$, we define the disordered model as the following probability measure $\mathrm{P}_{\Omega_{\delta} ; \lambda, h}^{\omega}$ for the field $\sigma=\left(\sigma_{x}\right)_{x \in \Omega_{\delta}}$ :

$$
\mathrm{P}_{\Omega_{\delta} ; \lambda, h}^{\omega}(\mathrm{d} \sigma):=\frac{e^{\sum_{x \in \Omega_{\delta}}\left(\lambda \omega_{x}+h\right) \sigma_{x}}}{Z_{\Omega_{\delta} ; \lambda, h}^{\omega}} \mathrm{P}_{\Omega_{\delta}}^{\mathrm{ref}}(\mathrm{d} \sigma),
$$

where the normalizing constant, called the partition function, is defined by

$$
Z_{\Omega_{\delta} ; \lambda, h}^{\omega}:=\mathrm{E}_{\Omega_{\delta}}^{\mathrm{ref}}\left[e^{\sum_{x \in \Omega_{\delta}}\left(\lambda \omega_{x}+h\right) \sigma_{x}}\right] .
$$

The quenched free energy $F(\lambda, h)$ of the model is defined as the rate of exponential growth of $Z_{\Omega_{\delta} ; \lambda, h}^{\omega}$ as $\Omega \uparrow \mathbb{R}^{d}$ for fixed $\delta$ (or equivalently $]^{\dagger}$ as $\delta \downarrow 0$ for fixed $\Omega$ ):

$$
F(\lambda, h):=\limsup _{\Omega \uparrow \mathbb{R}^{d}} \frac{1}{\left|\Omega_{\delta}\right|} \mathbb{E}\left[\log Z_{\Omega_{\delta} ; \lambda, h}^{\omega}\right]=\limsup _{\delta \downarrow 0} \frac{1}{\left|\Omega_{\delta}\right|} \mathbb{E}\left[\log Z_{\Omega_{\delta} ; \lambda, h}^{\omega}\right] .
$$

Discontinuities in the derivatives of the free energy correspond to phase transitions. A fundamental question is, does arbitrary disorder (i.e., $\lambda>0$ ) radically change the behavior

\footnotetext{
${ }^{\dagger}$ We assume the natural consistency condition that $\mathrm{P}_{(c \Omega) c \delta}^{\mathrm{ref}}$ coincides with $\mathrm{P}_{\Omega_{\delta}}^{\mathrm{ref}}$, for any $c>0$.
} 
of the homogeneous model (i.e., $\lambda=0$ ), such as qualitative properties of the law of the field $\sigma$ and/or the smoothness of the free energy in $h$ ? When the answer is affirmative, the model is called disorder relevant. In such cases, we will show that the disordered model typically admits a non-trivial scaling limit as $\delta \downarrow 0$, provided $\lambda, h \rightarrow 0$ at appropriate rates.

Informally speaking, our key assumption is that the discrete field $\sigma=\left(\sigma_{x}\right)_{x \in \Omega_{\delta}}$, under the reference law $\mathrm{P}_{\Omega_{\delta}}^{\text {ref }}$ and after a suitable rescaling, converges as $\delta \downarrow 0$ to a "continuum field" $\boldsymbol{\sigma}=\left(\boldsymbol{\sigma}_{x}\right)_{x \in \Omega}$, possibly distribution-valued, with law $\mathbf{P}_{\Omega}^{\text {ref }}$. (Our precise assumptions will be about the convergence of correlation functions, see (1.11).) Although the approach we follow is very general, we describe three specific models, to be discussed extensively in the sequel.

(1) The disordered pinning model $(d=1)$. Let $\tau=\left(\tau_{k}\right)_{k \geq 0}$ be a renewal process on $\mathbb{N}$ with $\mathrm{P}\left(\tau_{1}=n\right)=n^{-(1+\alpha)+o(1)}$, with $\alpha \in\left(\frac{1}{2}, 1\right)$. Take $\Omega=(0,1), \delta=\frac{1}{N}$ for $N \in \mathbb{N}$ and define $\mathrm{P}_{\Omega_{\delta}}^{\text {ref }}$ as the law of $\left(\sigma_{x}:=\mathbb{1}_{\delta \tau}(x)\right)_{x \in \Omega_{\delta}}$, where $\delta \tau=\left\{\frac{1}{N} \tau_{n}\right\}_{n \geq 0}$ is viewed as a random subset of $\Omega$. The continuum field $\mathbf{P}_{\Omega}^{\text {ref }}$ is $\left(\sigma_{x}=\mathbb{1}_{\boldsymbol{\tau}}(x)\right)_{x \in(0,1)}$ where $\boldsymbol{\tau}$ denotes the $\alpha$-stable regenerative set (the zero level set of a $\operatorname{Bessel}(2(1-\alpha))$ process).

(2) The (long-range) directed polymer model. Let $\left(S_{n}\right)_{n \geq 0}$ be a random walk on $\mathbb{Z}$ with i.i.d. increments, in the domain of attraction of an $\alpha$-stable law, with $1<\alpha \leq 2$. Take $\Omega=(0,1) \times \mathbb{R}, \delta=\frac{1}{N}$ for $N \in \mathbb{N}$ and, abusing notation, set $\Omega_{\delta}:=\left((\delta \mathbb{Z}) \times\left(\delta^{1 / \alpha} \mathbb{Z}\right)\right) \cap \Omega$. The "effective dimension" for this model is therefore $d_{\mathrm{eff}}:=1+\frac{1}{\alpha}$. Define $\mathrm{P}_{\Omega_{\delta}}^{\mathrm{ref}}$ as the law of the field $\left(\sigma_{x}:=\mathbb{1}_{A_{\delta}}(x)\right)_{x \in \Omega_{\delta}}$, where $A_{\delta}:=\left\{\left(\frac{n}{N}, \frac{S_{n}}{N^{1 / \alpha}}\right)\right\}_{n \geq 0}$ is viewed as a random subset of $\Omega$. The continuum field $\mathbf{P}_{\Omega}^{\text {ref }}$ is $\left(\boldsymbol{\sigma}_{x}=\mathbb{1}_{\boldsymbol{A}}(x)\right)_{x \in(0,1) \times \mathbb{R}}$ where $\boldsymbol{A}=\left\{\left(t, X_{t}\right)\right\}_{t \geq 0}$ and $\left(X_{t}\right)_{t \geq 0}$ is an $\alpha$-stable Lévy process (Brownian motion for $\alpha=2$ ).

(3) The random field Ising model $(d=2)$. Take any bounded and connected set $\Omega \subseteq \mathbb{R}^{2}$ with smooth boundary and define $\mathrm{P}_{\Omega_{\delta}}^{\text {ref }}$ to be the critical Ising model on $\Omega_{\delta}$ with inverse temperature $\beta=\beta_{c}=\frac{1}{2} \log (1+\sqrt{2})$ and + boundary condition. The (distribution valued) continuum field $\mathbf{P}_{\Omega}^{\text {ref }}$ has been recently constructed in [CGN12, CGN13, using breakthrough results on the scaling limit of correlation functions of the critical twodimensional Ising model, determined in [CHI12.

The restrictions on the dimensions and parameters of these models are linked precisely to the disorder relevance issue, as it will be explained later.

Since the reference law $\mathrm{P}_{\Omega_{\delta}}^{\text {ref }}$ has a weak limit $\mathbf{P}_{\Omega}^{\text {ref }}$ as $\delta \downarrow 0$, a natural question emerges: can one obtain a limit also for the disordered model $\mathrm{P}_{\Omega_{\delta} ; \lambda, h}^{\omega}$, under an appropriate scaling of the coupling constants $\lambda, h$ ? (We mean, of course, a non-trivial limit, which keeps track of $\lambda, h$; otherwise, it suffices to let $\lambda, h \rightarrow 0$ very fast to recover the "free case" $\mathbf{P}_{\Omega}^{\text {ref }}$.)

A natural strategy is to look at the exponential weight in (1.1). As $\delta \downarrow 0$ the discrete disorder $\omega=\left(\omega_{x}\right)_{x \in \Omega_{\delta}}$ approximates the white noise $W(\mathrm{~d} x)$, which is a sort of random signed measure on $\Omega$ (see Subsection 2.1 for more details). Then one might hope to define the candidate continuum disordered model $\mathbf{P}_{\Omega_{\delta} ; \hat{\lambda}, \hat{h}}^{W}$ by

$$
\frac{\mathrm{d} \mathbf{P}_{\Omega ; \hat{\lambda}, \hat{h}}^{W}}{\mathrm{~d} \mathbf{P}_{\Omega}^{\mathrm{ref}}}(\boldsymbol{\sigma}):=\frac{e^{\int_{\Omega} \boldsymbol{\sigma}_{x}(\hat{\lambda} W(\mathrm{~d} x)+\hat{h} \mathrm{~d} x)}}{\boldsymbol{Z}_{\Omega ; \hat{\lambda}, \hat{h}}^{W}},
$$

in analogy with (1.1), with $Z_{\Omega ; \hat{\lambda}, \hat{h}}^{W}$ defined accordingly, like in (1.2). 
Unfortunately, formula (1.4) typically makes no sense for $\hat{\lambda} \neq 0$, because the configurations of the continuum field $\boldsymbol{\sigma}=\left(\boldsymbol{\sigma}_{x}\right)_{x \in \Omega}$ under $\mathbf{P}_{\Omega}^{\text {ref }}$ are too rough or "thin" for the integral over $W(\mathrm{~d} x)$ to be meaningful (cf. the three motivating models listed above). We stress here that the difficulty is substantial and not just technical: for pinning and directed polymer models, one can show AKQ14b, CSZ14 that the scaling limit $\mathbf{P}_{\Omega ; \hat{\lambda}, \hat{h}}^{W}$ of $\mathrm{P}_{\Omega_{\delta} ; \lambda, h}^{\omega}$ exists, but for $\hat{\lambda} \neq 0$ it is not absolutely continuous with respect to $\mathbf{P}_{\Omega}^{\text {ref }}$. In particular, it is hopeless to define the continuum disordered model through a Radon-Nikodym density, like in (1.4).

1.2. General Strategy AND Results. In this paper we focus on the disordered partition function $Z_{\Omega_{\delta} ; \lambda, h}^{\omega}$. We show that, when $\delta \downarrow 0$ and $\lambda, h$ are scaled appropriately, the partition function admits a non-trivial limit in distribution, which is explicit and universal (i.e., it does not depend on the fine details of the model).

Switching from the random probability law $\mathrm{P}_{\Omega_{\delta} ; \lambda, h}^{\omega}$ to the random number $Z_{\Omega_{\delta} ; \lambda, h}^{\omega}$ is of course a simplification, whose relevance may not be evident. It turns out that the partition function contains the essential information on the model. In fact, the scaling limit of $Z_{\Omega_{\delta} ; \lambda, h}^{\omega}$, for sufficiently many domains $\Omega$ and "boundary conditions", allows to reconstruct the full scaling limit of $\mathrm{P}_{\Omega_{\delta} ; \lambda, h}^{\omega}$. This task has been achieved in AKQ14b for the directed polymer model based on simple random walk, and in CSZ14 for the disordered pinning model. We discuss the case of the long-range directed polymer model in Remark 3.9 below.

The scaling limit of $Z_{\Omega_{\delta} ; \lambda, h}^{\omega}$ can also describe the universal behavior of the free energy $F(\lambda, h)$ as $\lambda, h \rightarrow 0$, cf. Subsection 1.3. This explains the key role of the partition function and is a strong motivation for focusing on it in the first place.

We now describe our approach. The idea is to consider the so-called "high-temperature expansion" $(|\lambda|,|h| \ll 1)$ of the partition function $Z_{\Omega_{\delta} ; \lambda, h}^{\omega}$. When the field takes two values (say $\sigma_{x} \in\{0,1\}$, for simplicity), we can factorize and "linearize" the exponential in (1.2):

$$
Z_{\Omega_{\delta} ; \lambda, h}^{\omega}=\mathrm{E}_{\Omega_{\delta}}^{\mathrm{ref}}\left[\prod_{x \in \Omega_{\delta}}\left(1+\varepsilon_{x} \sigma_{x}\right)\right], \quad \text { where } \quad \varepsilon_{x}:=e^{\left(\lambda \omega_{x}+h\right)}-1 .
$$

Let us introduce the $k$-point correlation function $\psi_{\Omega_{\delta}}^{(k)}\left(x_{1}, \ldots, x_{k}\right)$ of the field under the reference law, defined for $k \in \mathbb{N}$ and distinct $x_{1}, \ldots, x_{k} \in \Omega$ by

$$
\psi_{\Omega_{\delta}}^{(k)}\left(x_{1}, \ldots, x_{k}\right):=\mathrm{E}_{\Omega_{\delta}}^{\mathrm{ref}}\left[\sigma_{x_{1}} \sigma_{x_{2}} \cdots \sigma_{x_{k}}\right]
$$

where we set $\sigma_{x}:=\sigma_{x_{\delta}}$ with $x_{\delta}$ being the point in $\Omega_{\delta}$ closest to $x \in \Omega$. (We define the correlation function on all points of $\Omega$ for later convenience, and set it to be zero whenever $\left(x_{i}\right)_{\delta}=\left(x_{j}\right)_{\delta}$ for some $i \neq j$.) A binomial expansion of the product in (1.5) then yields

$$
Z_{\Omega_{\delta} ; \lambda, h}^{\omega}=1+\sum_{k=1}^{\left|\Omega_{\delta}\right|} \frac{1}{k !} \sum_{\left(x_{1}, x_{2}, \ldots, x_{k}\right) \in\left(\Omega_{\delta}\right)^{k}} \psi_{\Omega_{\delta}}^{(k)}\left(x_{1}, \ldots, x_{k}\right) \prod_{i=1}^{k} \varepsilon_{x_{i}},
$$

where the $k$ ! accounts for the fact that we sum over ordered $k$-uples $\left(x_{1}, \ldots, x_{k}\right)$. We have rewritten the partition function as a multi-linear polynomial of the independent random variables $\left(\varepsilon_{x}\right)_{x \in \Omega_{\delta}}$ (what is called a polynomial chaos expansion), with coefficients given by the $k$-point correlation function of the reference field. Note that, by Taylor expansion,

$$
\mathbb{E}\left[\varepsilon_{x}\right] \simeq h+\frac{1}{2} \lambda^{2}=: h^{\prime}, \quad \operatorname{Var}\left[\varepsilon_{x}\right] \simeq \lambda^{2} .
$$

The crucial fact is that, for $|\lambda|,|h| \ll 1$, the distribution of a polynomial chaos expansion, like the right hand side of (1.7), is insensitive toward the marginal distribution of the random 
variables $\left(\varepsilon_{x}\right)_{x \in \Omega_{\delta}}$, as long as mean and variance are kept fixed. A precise formulation of this loosely stated invariance principle is given in Section 2, $c f$. Theorems [2.6] and [2.8, in the form of a Lindeberg principle. Denoting by $\left(\widetilde{\omega}_{x}\right)_{x \in \Omega_{\delta}}$ a family of i.i.d. standard Gaussians, by (1.8) we can then approximate

$$
Z_{\Omega_{\delta} ; \lambda, h}^{\omega} \simeq 1+\sum_{k=1}^{\left|\Omega_{\delta}\right|} \frac{1}{k !} \sum_{\left(x_{1}, x_{2}, \ldots, x_{k}\right) \in\left(\Omega_{\delta}\right)^{k}} \psi_{\Omega_{\delta}}^{(k)}\left(x_{1}, \ldots, x_{k}\right) \prod_{i=1}^{k}\left(\lambda \widetilde{\omega}_{x_{i}}+h^{\prime}\right) .
$$

Let us now introduce white noise $W(\cdot)$ on $\mathbb{R}^{d}$ (see Subsection 2.1): setting $\Delta:=\left(-\frac{\delta}{2}, \frac{\delta}{2}\right)^{d}$, we replace each $\widetilde{\omega}_{x}$ by $\delta^{-d / 2} W(x+\Delta)$. Since $h^{\prime}=h^{\prime} \delta^{-d} \operatorname{Leb}(x+\Delta)$, the inner sum in (1.9) coincides, up to boundary terms, with the following (deterministic + stochastic) integral:

$$
\int \cdots \int_{\Omega^{k}} \psi_{\Omega_{\delta}}^{(k)}\left(x_{1}, \ldots, x_{k}\right) \prod_{i=1}^{k}\left(\lambda \delta^{-d / 2} W\left(\mathrm{~d} x_{i}\right)+h^{\prime} \delta^{-d} \mathrm{~d} x_{i}\right) .
$$

(We recall that $\psi_{\Omega_{\delta}}^{(k)}\left(x_{1}, \ldots, x_{k}\right)$ is a piecewise constant function.)

We can now state our crucial assumption: we suppose that, for every $k \in \mathbb{N}$, there exist a symmetric function $\boldsymbol{\psi}_{\Omega}^{(k)}:\left(\mathbb{R}^{d}\right)^{k} \rightarrow \mathbb{R}$ and an exponent $\gamma \in[0, \infty)$ such that

$$
\left(\delta^{-\gamma}\right)^{k} \psi_{\Omega_{\delta}}^{(k)}\left(x_{1}, \ldots, x_{k}\right) \underset{\delta \downarrow}{\longrightarrow} \psi_{\Omega}^{(k)}\left(x_{1}, \ldots, x_{k}\right) \quad \text { in } L^{2}\left(\Omega^{k}\right) .
$$

By (1.10), if we fix $\hat{\lambda} \geq 0, \hat{h} \in \mathbb{R}$ and rescale the coupling constants as follows:

$$
\lambda=\hat{\lambda} \delta^{d / 2-\gamma}, \quad h^{\prime}=\hat{h} \delta^{d-\gamma} \quad\left(\text { where } h^{\prime}:=h+\frac{\lambda^{2}}{2}\right),
$$

equations (1.9)-(1.10) suggest that $Z_{\Omega_{\delta} ; \lambda, h}^{\omega}$ converges in distribution as $\delta \downarrow 0$ to a random variable which admits a Wiener chaos expansion with respect to the white noise $W(\cdot)$ :

$$
Z_{\Omega_{\delta} ; \lambda, h}^{\omega} \underset{\delta \downarrow 0}{\stackrel{d}{\longrightarrow}} Z_{\Omega ; \hat{\lambda}, \hat{h}}^{W}:=1+\sum_{k=1}^{\infty} \frac{1}{k !} \int \cdots \int_{\Omega^{k}} \boldsymbol{\psi}_{\Omega}^{(k)}\left(x_{1}, \ldots, x_{k}\right) \prod_{i=1}^{k}\left(\hat{\lambda} W\left(\mathrm{~d} x_{i}\right)+\hat{h} \mathrm{~d} x_{i}\right) .
$$

This is precisely what happens, as it follows from our main convergence results described in Section 2, . cf. Theorems 2.3 and 2.5. It is natural to call the random variable $Z_{\Omega ; \hat{\lambda}, \hat{h}}^{W}$ in (1.13) the continuum partition function, because it is the scaling limit of $Z_{\Omega_{\delta} ; \lambda, h}^{\omega}$.

Remark 1.1. The $L^{2}$ convergence in (1.11) typically imposes $\gamma<\frac{d}{2}$, cf. (1.16) below, which means that the disorder coupling constants $\lambda, h$ vanish as $\delta \downarrow 0$, by (1.12). The fact that the continuum partition function $\boldsymbol{Z}_{\Omega ; \hat{\lambda}, \hat{h}}^{W}$ in (1.13) is nevertheless a random object (for $\hat{\lambda}>0$ ) is a manifestation of disorder relevance. We elaborate more on this issue in Subsection 1.3.

Let us finally give a quick look at the three motivating models. The complete results are described in Section 3, $c f$. Theorems 3.1, 3.8 and 3.14. Note that the scaling exponents in (1.12) are determined by the dimension $d$ and by the exponent $\gamma$ appearing in (1.11).

(1) For the disordered pinning model $(d=1)$, one has $\gamma=1-\alpha$ by renewal theory [D97]. Relation (1.12) (for $\delta=\frac{1}{N}$ ) yields

$$
\lambda=\frac{\hat{\lambda}}{N^{\alpha-\frac{1}{2}}}, \quad h^{\prime}=\frac{\hat{h}}{N^{\alpha}} .
$$

Notice that $h^{\prime}=($ const. $) \lambda^{\frac{2 \alpha}{2 \alpha-1}}$ is precisely the scaling of the critical curve G10. 
(2) For the (long-range) directed polymer model $\left(d_{\mathrm{eff}}=1+\frac{1}{\alpha}\right)$, one has $\gamma=\frac{1}{\alpha}$ by Gnedenko's local limit theorem [BGT87. Therefore (1.12) (for $\delta=\frac{1}{N}$ and $d$ relpaced by $\left.d_{\text {eff }}\right)$ gives

$$
\lambda=\frac{\hat{\lambda}}{N^{\frac{\alpha-1}{2 \alpha}}}, \quad h^{\prime}=\frac{\hat{h}}{N} .
$$

(The parameter $h^{\prime}$ is actually irrelevant for this model, and one usually sets $h^{\prime} \equiv 0$, i.e., $h=-\frac{1}{2} \lambda^{2}$.) In the case $\alpha=2$, when the underlying random walk has zero mean and finite variance, one recovers the scaling $\lambda \approx N^{-1 / 4}$ determined in AKQ14a.

(3) For the random field Ising model $(d=2)$ one has $\gamma=\frac{1}{8}$ [CHI12, hence by (1.12)

$$
\lambda=\hat{\lambda} \delta^{\frac{7}{8}}, \quad h=\hat{h} \delta^{\frac{15}{8}} .
$$

(Note that $h$ instead of $h^{\prime}$ appears in this relation; moreover one should look at the normalized partition function $\exp \left(-\frac{1}{2} \lambda^{2}\left|\Omega_{\delta}\right|\right) Z_{\Omega_{\delta} ; \lambda, h}^{\omega}$. This is because $\sigma_{x} \in\{-1,+1\}$ instead of $\{0,1\}$, hence the starting relation (1.5) requires a correction.)

1.3. Discussion And perspectives. We now collect some comments and observations and point out some further directions of research.

1. (Disorder relevance). The main motivation of our approach is to understand the issue of disorder relevance, i.e. whether the addition of a small amount of disorder modifies the nature of the phase transition of the underlying homogeneous model. Remarkably, the key condition (1.11), which determines the class of models to which our approach applies, is consistent with the Harris criterion for disorder relevance, as we now discuss.

First we note that when $\gamma>0$, which is the most interesting case, condition (1.11) indicates that the reference law has polynomially decaying correlations, which is the signature that we are at the critical point of a continuous phase transition. In our context, this means that the order parameter $m_{h}:=\lim _{\delta \downarrow 0}\left|\Omega_{\delta}\right|^{-1} \mathrm{E}_{\Omega_{\delta} ; 0, h}\left[\sum_{x \in \Omega_{\delta}} \sigma_{x}\right]$ in the homogeneous model $(\lambda=0)$ vanishes continuously, but non-analytically, as $h \rightarrow 0$, cf. (1.1) and (1.2).

When (1.11) holds pointwise, with $\gamma>0$, the limiting correlation function typically diverges polynomially on diagonals, with the same exponent $\gamma$ :

$$
\psi_{\Omega}^{(k)}\left(x_{1}, \ldots, x_{k}\right) \approx\left\|x_{i}-x_{j}\right\|^{-\gamma} \quad \text { as } \quad x_{i} \rightarrow x_{j} .
$$

To have finite $L^{2}$ norm (which is necessary for $L^{2}$ convergence in (1.11)), such a local divergence must be locally square-integrable in $\mathbb{R}^{d}$ : this means that $(d-1)-2 \gamma>-1$, i.e.

$$
\gamma<\frac{d}{2}
$$

(Note that $\left(\frac{d}{2}-\gamma\right)$ is precisely the scaling exponent of the coupling constant $\lambda$ in (1.12).)

Relation (1.16) matches with the Harris criterion for disorder relevance [H74]. This was originally introduced in the context of the Ising model with bond disorder, but it can be naturally rephrased for general disordered system (cf. G10], CCFS86]): denoting by $\nu$ the correlation length exponent of the homogeneous system $(\lambda=0)$, it asserts that a $d$-dimensional system is disorder-relevant when $\nu<\frac{2}{d}$ and irrelevant when $\nu>\frac{2}{d}$ (the remaining case $\nu=\frac{2}{d}$ being dubbed marginal). The exponent $\nu$ is usually defined in terms of field correlations:

$$
\left|\mathrm{E}_{\Omega_{\delta} ; 0, h}\left(\sigma_{x} \sigma_{y}\right)-\mathrm{E}_{\Omega_{\delta} ; 0, h}\left(\sigma_{x}\right) \mathrm{E}_{\Omega_{\delta} ; 0, h}\left(\sigma_{y}\right)\right| \underset{\delta \downarrow 0}{\approx} e^{-\frac{|x-y|}{\delta \xi(h)}}, \quad \text { with } \quad \xi(h) \underset{h \downarrow 0}{\approx} h^{-\nu} .
$$


Since such an exponent can be difficult to compute, it is typical to consider alternative notions of correlation length $\xi(h)$, linked to finite size scaling [CCFS86, CCFS89]. In our context, it is natural to define

$$
\xi(h)^{-1}:=\max \left\{\delta>0: \quad Z_{\Omega_{\delta} ; 0, h}>A\right\},
$$

where $A>0$ is a fixed large constant, whose precise value is immaterial. Recalling (1.12), we can rewrite (1.13) for $\lambda=0$ as

$$
\lim _{h \downarrow 0} Z_{\Omega_{\delta} ; 0, h}=Z_{\Omega ; 0, \hat{h}}, \quad \text { with } \quad \delta=\delta_{h, \hat{h}}:=(h / \hat{h})^{1 /(d-\gamma)} .
$$

If $\hat{h} \mapsto \boldsymbol{Z}_{\Omega ; 0, \hat{h}}$ is increasing (e.g., when $\boldsymbol{\psi}_{\Omega}^{(k)}\left(x_{1}, \ldots, x_{k}\right) \geq 0$, by (1.13)) denoting by $\hat{h}_{A}$ the unique solution to $Z_{\Omega ; 0, \hat{h}}=A$, under some natural regularity assumptions it follows that

$$
\xi(h) \underset{h \downarrow 0}{\sim}\left(\delta_{h, \hat{h}_{A}}\right)^{-1}=\left(\frac{\hat{h}_{A}}{h}\right)^{1 /(d-\gamma)} \approx h^{-\nu}, \quad \text { with } \quad \nu=\frac{1}{d-\gamma} .
$$

This shows that Harris' condition $\nu<\frac{2}{d}$ coincides with the key condition $\gamma<\frac{d}{2}$ of our approach, cf. (1.16), ensuring the square-integrability of the limiting correlations.

The correlation length (1.18) is expected to be equivalent to the classical one (1.17), in the sense that it should have the same critical exponent (cf. [G07] for disordered pinning models) when the phase transition is continuous, that is when $\gamma>0$ in (1.15). When $\gamma=0$, which is the signature of a discontinuous (first-order) phase transition, our approach still applies and gives the scaling limit of the disorder partition function, but there is no direct link with disorder relevance (cf. (1.24) below and the following discussion).

In summary, our approach suggests an alternative view on disorder relevance, in which the randomness survives in the continuum limit with vanishing coupling constants. In fact, relation (1.13) can be seen as a rigorous finite size scaling relation Car88 for disordered systems (the special case of non-disordered pinning models is treated in [Soh09]).

Remark 1.2. We can now explain the parameter restrictions in the motivating models: condition (1.16) is fulfilled by the disordered pinning model $(d=1, \gamma=1-\alpha)$ when $\alpha>\frac{1}{2}$, by the (long-range) directed polymer model $\left(d_{\mathrm{eff}}=1+\frac{1}{\alpha}, \gamma=\frac{1}{\alpha}\right)$ when $\alpha>1$, and by the critical random field Ising model $\left(d=2, \gamma=\frac{1}{8}\right)$.

2. (Universality). The convergence in distribution of the discrete partition function $Z_{\Omega_{\delta} ; \lambda, h}^{\omega}$ toward its continuum counterpart $Z_{\Omega ; \hat{\lambda}, \hat{h}}^{W}$, cf. (1.13), is an instance of universality. In fact:

- the details of the disorder distribution are irrelevant: any family $\left(\omega_{x}\right)_{x \in \Omega_{\delta}}$ of i.i.d. random variables with zero mean, finite variance and locally finite exponential moments scales in the limit to the same continuum object, namely white noise $W(\cdot)$;

- also the fine details of the reference law $\mathrm{P}_{\Omega_{\delta}}^{\mathrm{ref}}$ disappear in the limit: any family $\psi_{\Omega_{\delta}}^{(k)}$ of discrete $k$-point correlation functions converging to the same limit (1.11) yields the same continuum partition function $Z_{\Omega ; \hat{\lambda}, \hat{h}}^{W}$ in (1.13).

At a deeper level, the continuum partition function sheds light on the discrete free energy $F(\lambda, h)$, cf. (1.3), in the weak disorder regime $\lambda, h \rightarrow 0$. Defining the continuum free energy

$$
\boldsymbol{F}(\hat{\lambda}, \hat{h}):=\limsup _{\Omega \uparrow \mathbb{R}^{d}} \frac{1}{\operatorname{Leb}(\Omega)} \mathbb{E}\left[\log \boldsymbol{Z}_{\Omega ; \hat{\lambda}, \hat{h}}^{W}\right]
$$


and setting $\lambda_{\delta}:=\delta^{d / 2-\gamma} \hat{\lambda}$ and $h_{\delta}:=\delta^{d-\gamma} \hat{h}-\frac{1}{2}\left(\lambda_{\delta}\right)^{2}$, cf. (1.12), one is led to the following

$$
\text { Conjecture : } \quad \lim _{\delta \downarrow 0} \frac{F\left(\lambda_{\delta}, h_{\delta}\right)}{\delta^{d}}=\boldsymbol{F}(\hat{\lambda}, \hat{h}) \text { 丹 }
$$

The heuristics goes as follows: by (1.3) we can write (replacing lim sup by lim for simplicity)

$$
\lim _{\delta \downarrow 0} \frac{F\left(\lambda_{\delta}, h_{\delta}\right)}{\delta^{d}}=\lim _{\delta \downarrow 0} \lim _{\Omega \uparrow \mathbb{R}^{d}} \frac{1}{\delta^{d}} \frac{1}{\left|\Omega_{\delta}\right|} \mathbb{E}\left[\log Z_{\Omega_{\delta} ; \lambda_{\delta}, h_{\delta}}^{\omega}\right] ;
$$

on the other hand, applying (1.13) in (1.19) (assuming uniform integrability) and noting that $\operatorname{Leb}(\Omega)=\lim _{\delta \downarrow 0} \delta^{d}\left|\Omega_{\delta}\right|$, one gets

$$
\boldsymbol{F}(\hat{\lambda}, \hat{h})=\lim _{\Omega \uparrow \mathbb{R}^{d}} \lim _{\delta \downarrow 0} \frac{1}{\delta^{d}} \frac{1}{\left|\Omega_{\delta}\right|} \mathbb{E}\left[\log Z_{\Omega_{\delta} ; \lambda_{\delta}, h_{\delta}}^{\omega}\right] .
$$

Therefore proving (1.20) amounts to interchanging the infinite volume $\left(\Omega \uparrow \mathbb{R}^{d}\right)$ and continuum and weak disorder $(\delta \downarrow 0)$ limits. This is in principle a delicate issue, but we expect relation (1.20) to hold in many interesting cases, such as the three motivating models in the specified parameters range (and, more generally, when the continuum correlations are "non trivial"; see the next point). This is an interesting open problem.

Relation (1.20) implies that the discrete free energy $F(\lambda, h)$ has a universal shape for weak disorder $\lambda, h \rightarrow 0$. This leads to sharp predictions on the asymptotic behavior of free energy-related quantities, such as critical curves and order parameters. Consider, e.g., the average magnetization $\left\langle\sigma_{0}\right\rangle_{\beta_{c}, h}$ in the critical Ising model on $\mathbb{Z}^{2}$ with a homogeneous external field $h>0$. If relation (1.20) holds (with $d=2, \lambda_{\delta}=\hat{\lambda} \delta^{\frac{7}{8}}, h_{\delta}=\hat{h} \delta^{\frac{15}{8}}$, cf. (1.14), and we look at the case $\hat{\lambda}=0$ ), differentiating both sides with respect to $\hat{h}$ suggests that

$$
\lim _{h \downarrow 0} \frac{\left\langle\sigma_{0}\right\rangle_{\beta_{c}, h}}{h^{\frac{1}{15}}}=\frac{\partial \boldsymbol{F}}{\partial \hat{h}}(0,1),
$$

which would sharpen the results in [CGN12b. Analogous predictions can be formulated for disorder pinning and directed polymer models (see Section 3). Of course, proving such precise estimates is likely to require substantial additional work, but having a candidate for the limiting constants, like in (1.23), can be of great help.

Remark 1.3. Relation (1.20) (in a stronger form) has been proved in [BdH97, CG10] for the so-called disordered copolymer model, by means of a subtle coarse-graining procedure. We mention that our approach can also be applied to the copolymer model, yielding a Wiener chaos expansion as in (1.13) for the continuum partition function.

3. (First-order phase transitions). Relation (1.11) can hold with $\gamma=0$ (i.e., the $k$-point correlation function converges without rescaling) and with a "trivial" factorized limit:

$$
\psi_{\Omega_{\delta}}^{(k)}\left(x_{1}, \ldots, x_{k}\right) \underset{\delta \downarrow 0}{\longrightarrow} \psi_{\Omega}^{(k)}\left(x_{1}, \ldots, x_{k}\right):=\varrho^{k}, \quad \text { with } \varrho \in(0, \infty) .
$$

This is typical for a system at the critical point of a first-order phase transitions (i.e., the order parameter $m_{h}:=\lim _{\delta \downarrow 0}\left|\Omega_{\delta}\right|^{-1} \mathrm{E}_{\Omega_{\delta} ; 0, h}\left[\sum_{x \in \Omega_{\delta}} \sigma_{x}\right]$, as a function of $h$, has a jump discontinuity at $h=0$ ). Examples include the pinning model for $\alpha>1$ and the Ising model

\footnotetext{
${ }^{\dagger}$ A notational remark: for the directed polymer model, the denominator in (1.20) should be $\delta$ instead of $\delta^{d}$, due to a different normalization of the discrete free energy, cf. Subsection 3.2 for the random field Ising model, one should set $h_{\delta}:=\delta^{d-\gamma} \hat{h}$ without the " $-\frac{1}{2}\left(\lambda_{\delta}\right)^{2}$ correction", as already discussed after (1.14). These notational details are discussed in Section 3 for each model, while here we keep a unified approach.
} 
for $\beta>\beta_{c}$. Plugging (1.24) into (1.13) and performing the integration, cf. [J97, §3.2], one gets

$$
Z_{\Omega ; \hat{\lambda}, \hat{h}}^{W}=\exp \left\{\varrho \hat{\lambda} W(\Omega)+\left(\varrho \hat{h}-\frac{1}{2}(\varrho \hat{\lambda})^{2}\right) \operatorname{Leb}(\Omega)\right\} .
$$

This explicit formula allows exact asymptotic computations on the discrete model: e.g., relation (1.13) yields for suitable values of $\zeta \in \mathbb{R}$ (when uniform integrability holds)

$$
\mathbb{E}\left[\left(Z_{\Omega_{\delta} ; \lambda, h}^{\omega}\right)^{\zeta}\right] \underset{\delta \downarrow 0}{\longrightarrow} \mathbb{E}\left[\left(Z_{\Omega ; \hat{\lambda}, \hat{h}}^{W}\right)^{\zeta}\right]=\exp \left\{\varrho \zeta\left(\hat{h}-\frac{1}{2} \varrho \hat{\lambda}^{2}(1-\zeta)\right) \operatorname{Leb}(\Omega)\right\} .
$$

Incidentally, for disordered pinning models with $\alpha>1$, this estimate clarifies the strategy for the sharp asymptotic behavior of the critical curve $h_{c}(\lambda)$ as $\lambda \downarrow 0$, determined in [BCPSZ14] (even though the proof therein is carried out with different techniques).

Unfortunately, the continuum partition function (1.25) can fail in capturing some key properties of the discrete model, because it is shared by many "too different" models: relation (1.24) asks that the field variables under the reference law $\mathrm{P}^{\mathrm{ref}}$ become uncorrelated as $\delta \downarrow 0$, but is insensitive toward the correlation decay, which could be polynomial, exponential, or even finite-range (like in the extreme case of a "trivial" reference law $\mathrm{P}^{\text {ref }}$, under which $\left(\sigma_{x}\right)_{x \in \Omega_{\delta}}$ are i.i.d. with $\left.\mathrm{E}^{\mathrm{ref}}\left(\sigma_{x}\right)=\varrho\right)$. Since the correlation decay can affect substantially the discrete free energy, conjecture (1.20) usually fails under (1.24).

For example, for disordered pinning models with $\alpha>1$, one always has $F(\lambda, h) \geq 0$ (there is only a polynomial cost for the underlying renewal process not to return before time $N$, and the energy of such a renewal configuration is 0 ). On the other hand, if the renewal jump distribution has finite exponential moments, then there is an exponential cost for the renewal not to return before time $N$, and $F(\lambda, h)<0$ if $h$ is sufficiently negative. Both models satisfy (1.24) with $\varrho=1 / \mathrm{E}\left[\tau_{1}\right]$ and thus their continuum partition functions coincide, but their free energies depend on finer detail of the renewal distribution (beyond the value of $\left.\mathrm{E}\left[\tau_{1}\right]\right)$ and are therefore radically different, causing (1.20) to fail. The continuum free energy is $\boldsymbol{F}(\hat{\lambda}, \hat{h})=\varrho \hat{h}-\frac{1}{2}(\varrho \hat{\lambda})^{2}$, cf. (1.25) and (1.19), which can attain negative values.

4. (Moment assumptions). In our convergence results, cf. Theorems 3.1 3.8 and 3.14, we assume that the disorder variables $\left(\omega_{x}\right)_{x \in \Omega_{\delta}}$ have finite exponential moments, which guarantees that the expectation and variance in (1.8) are well-defined. However, this assumption can be relaxed to finite moments. The necessary number of moments depends on the model and can be determined by the requirement that the typical maximum value of the variables $\omega_{x}$ "sampled" by the field does not exceed the reciprocal of the disorder strength $\lambda$ (so that a truncation of $\omega_{x}$ at level $\lambda^{-1}$ provides a good approximation).

For example, in the long-range directed polymer model, one expects that the path will be confined (at weak disorder) in a box of size $N \times N^{1 / \alpha}$. If the disorder variables have a polynomial tail $\mathbb{P}\left(\omega_{x}>y\right) \approx y^{-\eta}$ as $y \uparrow \infty$, their maximum in such a box is of the order $N^{\frac{1}{\eta} \frac{1+\alpha}{\alpha}}$. Since $\lambda \approx N^{-\frac{\alpha-1}{2 \alpha}}$ for this model, cf. Theorem 3.8, one expects the validity of the convergence result as long as $N^{\frac{1}{\eta} \frac{1+\alpha}{\alpha}} \ll N^{\frac{\alpha-1}{2 \alpha}}$, i.e. for $\eta \geq 2(\alpha+1) /(\alpha-1)$. For $\alpha=2$, this gives $\eta \geq 6$, which was conjectured in AKQ14a] and recently proved in [DZ].

Similarly, for the pinning model the number of relevant variables is of order $N$ and $\lambda \approx N^{-\left(\alpha-\frac{1}{2}\right)}$, cf. Theorem 3.1, leading to a conjectured value $\eta \geq 2 /(2 \alpha-1)$; for the RFIM, the number of relevant variables is of order $N^{2}$ and $\lambda \approx N^{-7 / 8}$, cf. Theorem 3.14, leading to a conjectured value $\eta \geq 16 / 7$. 


\section{From polynomial to Wiener chaos via Lindeberg}

In this section, which can be read independently of the previous one, we first recall the main properties of white noise on $\mathbb{R}^{d}$ (Subsection 2.1) and define polynomial chaos expansions (Subsection 2.2). We then formulate our main general theorem (Subsection 2.3), ensuring convergence of polynomial chaos toward Wiener chaos expansions. This is based on a Lindeberg principle (Subsection 2.4) which extends results in MOO10 to optimal second moment assumptions. Subsections 2.3 and 2.4 can be read independently.

The space of Lebesgue square-integrable functions $f: \mathbb{R}^{d} \rightarrow \mathbb{R}$ is denoted by $L^{2}\left(\mathbb{R}^{d}\right)$, and we set $\|f\|_{L^{2}\left(\mathbb{R}^{d}\right)}^{2}=\int_{\mathbb{R}^{d}} f(x)^{2} \mathrm{~d} x$. For more details on the white noise, we refer to [J97, PT10].

2.1. White Noise IN a Nutshell. By white noise on $\mathbb{R}^{d}$ we mean a Gaussian process $W=(W(f))_{f \in L^{2}\left(\mathbb{R}^{d}\right)}$ with $\mathbb{E}[W(f)]=0$ and $\operatorname{Cov}(W(f), W(g))=\int_{\mathbb{R}^{d}} f(x) g(x) \mathrm{d} x$, defined on some probability space $\left(\Omega_{W}, \mathcal{A}, \mathbb{P}\right)$. Since the specified covariance is a symmetric and positive definite function, such a process exists (and is unique in law).

If $A_{1}, A_{2}, \ldots$ are disjoint Borel sets with finite Lebesgue measure, it follows that the random variables $\left(W\left(A_{i}\right):=W\left(\mathbb{1}_{A_{i}}\right)\right)_{i=1,2, \ldots}$ are independent $\mathcal{N}\left(0, \operatorname{Leb}\left(A_{i}\right)\right)$ and the relation $W\left(\bigcup_{i \geq 1} A_{i}\right)=\sum_{i \geq 1} W\left(A_{i}\right)$ holds a.s.. Consequently, it is suggestive to use the notation

$$
\int_{\mathbb{R}^{d}} f(x) W(\mathrm{~d} x):=W(f),
$$

even though $W(\cdot)$ is a.s. not a signed measure on $\mathbb{R}^{d}$. For $d=1, W(f)$ coincides with the usual Wiener integral $\int f(t) \mathrm{d} W_{t}$ with respect to the Brownian motion $W_{t}:=W\left(\mathbb{1}_{[0, t]}\right)$.

One can define a multi-dimensional stochastic integral $W^{\otimes k}(f)$, for $k \in \mathbb{N}$ and suitable $f:\left(\mathbb{R}^{d}\right)^{k} \rightarrow \mathbb{R}$, as follows. For "special indicator functions" $f=\mathbb{1}_{A_{1} \times \ldots \times A_{k}}$ built over disjoint bounded Borel sets $A_{1}, \ldots, A_{k} \subseteq \mathbb{R}^{d}$, one poses $W^{\otimes k}(f):=W\left(\mathbb{1}_{A_{1}}\right) \cdots W\left(\mathbb{1}_{A_{k}}\right)$. This definition is extended, by linearity, to the space $\mathcal{S}_{k}$ of "special simple functions", i.e. finite linear combinations of special indicator functions. Since a permutation of the arguments of $f$ leaves $W^{\otimes k}(f)$ invariant, it is sufficient to consider symmetric functions $f$, which we do henceforth. One then observes that $\mathbb{E}\left[W^{\otimes k}(f)\right]=0$ and the crucial Ito isometry is satisfied

$$
\operatorname{Cov}\left(W^{\otimes k}(f), W^{\otimes l}(g)\right)=k ! \mathbb{1}_{\{k=l\}} \int_{\left(\mathbb{R}^{d}\right)^{k}} f\left(x_{1}, \ldots, x_{k}\right) g\left(x_{1}, \ldots, x_{k}\right) \mathrm{d} x_{1} \cdots \mathrm{d} x_{k} .
$$

Since $\mathcal{S}_{k}$ is dense in $L^{2}\left(\left(\mathbb{R}^{d}\right)^{k}\right)$, one can finally extend the definition of $W^{\otimes k}(f)$ to every symmetric $f \in L^{2}\left(\left(\mathbb{R}^{d}\right)^{k}\right)$, in such a way that (2.2) still holds. Like in (2.1), we will write

$$
\int \cdots \int_{\left(\mathbb{R}^{d}\right)^{k}} f\left(x_{1}, \ldots, x_{k}\right) W\left(\mathrm{~d} x_{1}\right) \cdots W\left(\mathrm{~d} x_{k}\right):=W^{\otimes k}(f) .
$$

One also sets $W^{\otimes 0}(c):=c$ for $c \in L^{2}\left(\left(\mathbb{R}^{d}\right)^{0}\right):=\mathbb{R}$.

Note that $W^{\otimes k}(f)$ is a random variable defined on $\left(\Omega_{W}, \mathcal{A}, \mathbb{P}\right)$, with zero mean (for $k \geq 1$ ) and finite variance, which is measurable with respect to the $\sigma$-algebra $\sigma(W)$ generated by the white noise $W$. (One can show that $W^{\otimes k}(f)$ is non Gaussian for $k>1$ and $f \not \equiv 0$.) Remarkably, every square-integrable random variable $X$ defined on $\Omega_{W}$, which is measurable with respect to $\sigma(W)$, can be written as the $L^{2}$-convergent series

$$
X=\sum_{k=0}^{\infty} \frac{1}{k !} W^{\otimes k}\left(f_{k}\right),
$$

\footnotetext{
†For the isometry (2.2) it is essential to "avoid diagonals": this is the reason for taking special indicator functions, corresponding to products of disjoint Borel sets.
} 
called Wiener chaos expansion, for a unique choice of symmetric functions $f_{k} \in L^{2}\left(\left(\mathbb{R}^{d}\right)^{k}\right)$ satisfying $\sum_{k=0}^{\infty} \frac{1}{k !}\left\|f_{k}\right\|_{L^{2}\left(\mathbb{R}^{d}\right)}^{2}<\infty$, by (2.2). In other terms, the multiple stochastic integrals $W^{\otimes k}(f)$ span the whole Hilbert space $L^{2}\left(\Omega_{W}, \sigma(W), \mathbb{P}\right)$.

2.2. Polynomial Chaos. Let $\mathbb{T}$ be a finite or countable index set (e.g., $\mathbb{T}=\{1, \ldots, N\}$, $\mathbb{T}=\mathbb{N}, \mathbb{T}=\mathbb{Z}^{d}$ ). We set

$$
\mathcal{P}^{\text {fin }}(\mathbb{T}):=\{I \subseteq \mathbb{T}:|I|<\infty\} .
$$

Any function $\psi: \mathcal{P}^{\mathrm{fin}}(\mathbb{T}) \rightarrow \mathbb{R}$ determines a (formal, if $|\mathbb{T}|=\infty$ ) multi-linear polynomial $\Psi$ :

$$
\Psi(x)=\sum_{I \in \mathcal{P} \text { fin }(\mathbb{T})} \psi(I) x^{I}, \quad \text { where } \quad x^{I}:=\prod_{i \in I} x_{i} \quad \text { with } \quad x^{\emptyset}:=1 .
$$

We say that $\psi: \mathcal{P}^{\text {fin }}(\mathbb{T}) \rightarrow \mathbb{R}$ is the kernel of $\Psi$.

Let now $\zeta:=\left(\zeta_{i}\right)_{i \in \mathbb{T}}$ be a family of independent (but not necessarily identically distributed) random variables. We say that a random variable $X$ admits a polynomial chaos expansion with respect to $\zeta$ if it can be expressed as $X=\Psi(\zeta)=\Psi\left(\left(\zeta_{i}\right)_{i \in \mathbb{T}}\right)$ for some multi-linear polynomial $\Psi$. Of course, when $|\mathbb{T}|=\infty$ some care is needed: by $X=\Psi(\zeta)$ we mean that for any sequence $\Lambda_{N} \subset \mathbb{T}$ with $\left|\Lambda_{N}\right|<\infty$ and $\Lambda_{N} \uparrow \mathbb{T}$ one has

$$
X=\lim _{N \rightarrow \infty} \sum_{I \subseteq \Lambda_{N}} \psi(I) \zeta^{I} \quad \text { in probability } .
$$

Remark 2.1. When $\operatorname{Var}\left(\zeta_{i}\right) \neq 0$ for all $i \in \mathbb{T}$, we can assume that all the variances are equal with no loss of generality: it suffices to redefine $\psi(I) \rightarrow \psi(I)\left(\prod_{i \in I} \operatorname{Var}\left(\zeta_{i}\right)\right)^{-1 / 2}$.

Remark 2.2. When the independent random variables $\zeta:=\left(\zeta_{i}\right)_{i \in \mathbb{T}}$ have zero mean and the same variance $\sigma^{2}$, an easy sufficient condition for (2.6), with $L^{2}$ convergence, is

$$
\sum_{I \in \mathcal{P}^{\text {fin }}(\mathbb{T})}\left(\sigma^{2}\right)^{|I|} \psi(I)^{2}<\infty
$$

because $\mathbb{E}\left[\zeta^{I} \zeta^{J}\right]=0$ for $I \neq J$. For variables with non-zero mean $\mu:=\left(\mu_{i}\right)_{i \in \mathbb{T}}$ (always with the same variance $\sigma^{2}$ ), sharp conditions for $L^{2}$ convergence in (2.6) involve $\mu$ and the kernel $\psi$ jointly. As we show below, practical sufficient "factorized" conditions are

$$
\sum_{i \in \mathbb{T}} \mu_{i}^{2}<\infty ; \quad \exists \varepsilon>0: \quad \sum_{I \in \mathcal{P}^{\text {fin }}(\mathbb{T})}(1+\varepsilon)^{|I|}\left(\sigma^{2}\right)^{|I|} \psi(I)^{2}<\infty .
$$

2.3. Convergence of polynomial chaos to Wiener chaos. Consider for $\delta \in(0,1)$ an index set $\mathbb{T}_{\delta} \subset \mathbb{R}^{d}$ and a family of polynomial chaos expansions $\left(\Psi_{\delta}\left(\zeta_{\delta}\right)\right)_{\delta \in(0,1)}$, defined from kernels $\psi_{\delta}: \mathcal{P}^{\text {fin }}\left(\mathbb{T}_{\delta}\right) \rightarrow \mathbb{R}$ and from independent random variables $\zeta_{\delta}:=$ $\left(\zeta_{\delta, x}\right)_{x \in \mathbb{T}_{\delta}}$. If $\mathbb{T}_{\delta}$ converges to the continuum $\mathbb{R}^{d}$ as $\delta \downarrow 0$ (e.g., $\left.\mathbb{T}_{\delta}:=(\delta \mathbb{Z})^{d}\right)$, then after suitable scaling, the random variables $\left(\zeta_{\delta, x}\right)_{x \in \mathbb{T}_{\delta}}$ approximate the white noise $W(\mathrm{~d} x)$ on $\mathbb{R}^{d}$. If the kernel $\psi_{\delta}$, suitably rescaled, converges as $\delta \downarrow 0$ to a continuum kernel $\boldsymbol{\psi}_{0}: \mathcal{P}^{\text {fin }}\left(\mathbb{R}^{d}\right) \rightarrow$ $\mathbb{R}$, it is plausible that the polynomial chaos expansion $\Psi_{\delta}\left(\zeta_{\delta}\right)$ approximates a Wiener chaos expansion $\boldsymbol{\Psi}_{0}$, cf. (2.3)-(2.4), with kernel $\boldsymbol{\psi}_{0}$. This is precisely what we are going to show.

First we introduce some notation. Each random variable $\zeta_{x}$ indexed by a point $x$ in an index set $\mathbb{T} \subset \mathbb{R}^{d}$ will be associated with a cell in $\mathbb{R}^{d}$ containing $x$, and functions defined on $\mathbb{T}^{k}$ will be extended to functions defined on $\left(\mathbb{R}^{d}\right)^{k}$.

- Let $\mathcal{B}\left(\mathbb{R}^{d}\right)$ denote the Borel subsets of $\mathbb{R}^{d}$. Given a locally finite set $\mathbb{T} \subset \mathbb{R}^{d}$, we call $\mathcal{C}: \mathbb{T} \rightarrow \mathcal{B}\left(\mathbb{R}^{d}\right)$ a tessellation of $\mathbb{R}^{d}$ indexed by $\mathbb{T}$, if $(\mathcal{C}(x))_{x \in \mathbb{T}}$ form a disjoint partition 
of $\mathbb{R}^{d}$ such that $x \in \mathcal{C}(x)$ for each $x \in \mathbb{T}$. We call $\mathcal{C}(x)$ the cell associated with $x \in \mathbb{T}$. In most cases, $(\mathcal{C}(x))_{x \in \mathbb{T}}$ will be the cells of a cubic lattice. However, there are natural examples where this is not the case, such as the directed polymer model defined from a simple symmetric random walk, or the Ising model defined on non-cubic lattices.

- Once a tessellation $\mathcal{C}$ is fixed, any function $f: \mathbb{T} \rightarrow \mathbb{R}$ is automatically extended to $f: \mathbb{R}^{d} \rightarrow \mathbb{R}$ by assigning value $f(y):=f(x)$ for all $y \in \mathcal{C}(x)$, for each $x \in \mathbb{T}$. Note that for such extensions $\|f\|_{L^{2}\left(\mathbb{R}^{d}\right)}^{2}=\sum_{x \in \mathbb{T}} f(x)^{2} \operatorname{Leb}(\mathcal{C}(x))$.

- Analogously, for any $\psi: \mathcal{P}^{\text {fin }}(\mathbb{T}) \rightarrow \mathbb{R}$, we first extend it to $\psi: \bigcup_{k=0}^{\infty} \mathbb{T}^{k} \rightarrow \mathbb{R}$ by setting $\psi\left(x_{1}, \ldots, x_{k}\right):=\psi\left(\left\{x_{1}, \ldots, x_{k}\right\}\right)$ if the $x_{i}$ are distinct, and $\psi\left(x_{1}, \ldots, x_{k}\right):=0$ otherwise. We then extend it to $\psi: \bigcup_{k=0}^{\infty}\left(\mathbb{R}^{d}\right)^{k} \rightarrow \mathbb{R}$ by assigning value $\psi\left(x_{1}, \ldots, x_{k}\right)$ to all points in $\mathcal{C}\left(x_{1}\right) \times \cdots \times \mathcal{C}\left(x_{k}\right)$, for each $k \in \mathbb{N}$ and $x_{1}, \ldots, x_{k} \in \mathbb{T}$.

- Given $\psi: \mathcal{P}^{\text {fin }}\left(\mathbb{R}^{d}\right) \rightarrow \mathbb{R}$, its extension to $\psi: \bigcup_{k=0}^{\infty}\left(\mathbb{R}^{d}\right)^{k} \rightarrow \mathbb{R}$ is defined similarly (no cells involved). It will be clear from the context which version of $\psi$ is being used.

- Finally, given a measurable function $\psi: \bigcup_{k=0}^{\infty}\left(\mathbb{R}^{d}\right)^{k} \rightarrow \mathbb{R}$, we denote by $\|\psi\|_{L^{2}\left(\left(\mathbb{R}^{d}\right)^{k}\right)}$ the $L^{2}$ norm of the restriction of $\psi$ to $\left(\mathbb{R}^{d}\right)^{k}$, i.e.

$$
\|\psi\|_{L^{2}\left(\left(\mathbb{R}^{d}\right)^{k}\right)}^{2}=\int \cdots \int_{\left(\mathbb{R}^{d}\right)^{k}} \psi\left(x_{1}, \ldots, x_{k}\right)^{2} \mathrm{~d} x_{1} \cdots \mathrm{d} x_{k} .
$$

We are now ready to state our main convergence result, proved in Section 5 ,

Theorem 2.3 (Convergence of polynomial chaos to Wiener chaos, $L^{2}$ case). Assume that for $\delta \in(0,1)$ the following ingredients are given:

- Let $\mathbb{T}_{\delta}$ be a locally finite subset of $\mathbb{R}^{d}$;

- Let $\zeta_{\delta}:=\left(\zeta_{\delta, x}\right)_{x \in \mathbb{T}_{\delta}}$ be independent random variables in $L^{2}$ with the same variance,

$$
\mathbb{E}\left[\zeta_{\delta, x}\right]=\mu_{\delta}(x) \quad \text { and } \quad \operatorname{Var}\left(\zeta_{\delta, x}\right)=\sigma_{\delta}^{2},
$$

such that $\left(\left(\zeta_{\delta, x}-\mathbb{E}\left[\zeta_{\delta, x}\right]\right)^{2}\right)_{\delta \in(0,1), x \in \mathbb{T}_{\delta}}$ are uniformly integrable;

- Let $\Psi_{\delta}(z)$ be a formal multi-linear polynomial with kernel $\psi_{\delta}: \mathcal{P}^{\text {fin }}\left(\mathbb{T}_{\delta}\right) \rightarrow \mathbb{R}$, cf. (2.5) ;

- Let $\mathcal{C}_{\delta}$ be a tessellation of $\mathbb{R}^{d}$ indexed by $\mathbb{T}_{\delta}$, where every cell $\mathcal{C}_{\delta}(x)$ has the same volume $v_{\delta}:=\operatorname{Leb}\left(\mathcal{C}_{\delta}(x)\right)$.

Assume that $v_{\delta} \rightarrow 0$ as $\delta \downarrow 0$, and that the following conditions are satisfied:

(i) There exist $\boldsymbol{\sigma}_{0} \in(0, \infty)$ and $\boldsymbol{\mu}_{0} \in L^{2}\left(\mathbb{R}^{d}\right)$ such that

$$
\lim _{\delta \downarrow 0} \sigma_{\delta}=\boldsymbol{\sigma}_{0}, \quad \lim _{\delta \downarrow 0}\left\|\bar{\mu}_{\delta}-\boldsymbol{\mu}_{0}\right\|_{L^{2}\left(\mathbb{R}^{d}\right)}=0, \quad \text { where } \quad \bar{\mu}_{\delta}(x):=v_{\delta}^{-1 / 2} \mu_{\delta}(x) ;
$$

(ii) There exists $\boldsymbol{\psi}_{0}: \mathcal{P}^{\text {fin }}\left(\mathbb{R}^{d}\right) \rightarrow \mathbb{R}$, with $\left\|\boldsymbol{\psi}_{0}\right\|_{L^{2}\left(\left(\mathbb{R}^{d}\right)^{k}\right)}<\infty$ for every $k \in \mathbb{N}_{0}$, such that

$$
\lim _{\delta \downarrow 0}\left\|\bar{\psi}_{\delta}-\boldsymbol{\psi}_{0}\right\|_{L^{2}\left(\left(\mathbb{R}^{d}\right)^{k}\right)}^{2}=0, \quad \text { where } \bar{\psi}_{\delta}(I):=v_{\delta}^{-|I| / 2} \psi_{\delta}(I)
$$

(iii) For some $\varepsilon>0$ (or even $\varepsilon=0$, if $\mu_{\delta}(x) \equiv 0$ )

$$
\lim _{\ell \rightarrow \infty} \limsup _{\delta \downarrow 0} \sum_{I \in \mathcal{P}_{\text {fin }}\left(\mathbb{T}_{\delta}\right),|I|>\ell}(1+\varepsilon)^{|I|}\left(\sigma_{\delta}^{2}\right)^{|I|} \psi_{\delta}(I)^{2}=0 .
$$


Then the polynomial chaos expansion $\Psi_{\delta}\left(\zeta_{\delta}\right)$ is well-defined and converges in distribution as $\delta \downarrow 0$ to a random variable $\boldsymbol{\Psi}_{0}$ with an explicit Wiener chaos expansion:

$$
\Psi_{\delta}\left(\zeta_{\delta}\right) \underset{\delta \downarrow 0}{\stackrel{d}{\longrightarrow}} \Psi_{0}:=\sum_{k=0}^{\infty} \frac{1}{k !} \int \cdots \int_{\left(\mathbb{R}^{d}\right)^{k}} \boldsymbol{\psi}_{0}\left(y_{1}, \ldots, y_{k}\right) \prod_{i=1}^{k}\left(\boldsymbol{\sigma}_{0} W\left(\mathrm{~d} y_{i}\right)+\boldsymbol{\mu}_{0}\left(y_{i}\right) \mathrm{d} y_{i}\right)
$$

where $W(\cdot)$ denotes white noise on $\mathbb{R}^{d}$.

The series in (2.12) converges in $L^{2}$, and $\mathbb{E}\left[\Psi_{\delta}\left(\zeta_{\delta}\right)^{2}\right] \rightarrow \mathbb{E}\left[\Psi_{0}^{2}\right]$. Consequently, for any coupling of $\Psi_{\delta}\left(\zeta_{\delta}\right)$ and $\boldsymbol{\Psi}_{0}$ such that $\Psi_{\delta}\left(\zeta_{\delta}\right) \rightarrow \boldsymbol{\Psi}_{0}$ a.s., one has $\mathbb{E}\left[\left|\Psi_{\delta}\left(\zeta_{\delta}\right)-\boldsymbol{\Psi}_{0}\right|^{2}\right] \rightarrow 0$.

The convergence (2.12) extends to the joint distribution of a finite collection of polynomial chaos expansions $\left(\Psi_{i, \delta}\left(\zeta_{\delta}\right)\right)_{1 \leq i \leq M}$, provided $\left(\Psi_{i, \delta}\right)_{\delta \in(0,1)}$ satisfies (iii)-(iii) above for each $i$.

Remark 2.4. Let us be more precise about the random variable $\Psi_{0}$ in (2.12). Setting $\boldsymbol{\nu}(x):=\boldsymbol{\mu}_{0}(x) / \boldsymbol{\sigma}_{0}$, it can be rewritten as

$$
\mathbf{\Psi}_{0}=\sum_{k=0}^{\infty} \frac{1}{k !} \int \cdots \int_{\left(\mathbb{R}^{d}\right)^{k}} \boldsymbol{\psi}_{0}\left(y_{1}, \ldots, y_{k}\right) \boldsymbol{\sigma}_{0}^{k} \prod_{i=1}^{k}\left(W\left(\mathrm{~d} y_{i}\right)+\boldsymbol{\nu}\left(y_{i}\right) \mathrm{d} y_{i}\right),
$$

which can be viewed as a "Wiener chaos expansion with respect to the biased white noise $W_{\boldsymbol{\nu}}(\mathrm{d} x):=W(\mathrm{~d} x)+\boldsymbol{\nu}(x) \mathrm{d} x "$. The rigorous definition of such an expansion goes as follows. For every fixed $k \in \mathbb{N}$, the integral over $\left(\mathbb{R}^{d}\right)^{k}$ in $(2.13)$ can be defined by expanding the product and integrating out the "deterministic coordinates" (those corresponding to $\left.\boldsymbol{\nu}\left(y_{i}\right) \mathrm{d} y_{i}\right)$, obtaining a finite sum of well-defined (lower-dimensional) ordinary stochastic integrals, like in (2.3). Regrouping the terms, the series in (2.13) becomes an ordinary Wiener chaos expansion, like in (2.4). In analogy with the polynomial case (2.8), we show in Section 5 that the $L^{2}$-convergence of the series is ensured by the conditions that $\boldsymbol{\mu}_{0} \in L^{2}\left(\mathbb{R}^{d}\right)$ and that

$$
\exists \varepsilon>0: \quad \sum_{k=0}^{\infty} \frac{1}{k !}(1+\varepsilon)^{k}\left(\boldsymbol{\sigma}_{0}^{2}\right)^{k}\left\|\boldsymbol{\psi}_{0}\right\|_{L^{2}\left(\left(\mathbb{R}^{d}\right)^{k}\right)}^{2}<\infty,
$$

which follow by assumptions (1i)-(iii)-(iii) in Theorem 2.3 .

2.3.1. Beyond the $L^{2}$ CASE. There is a useful alternative interpretation of (2.12)-(2.13) . If $\left(\Omega_{W}, \mathcal{A}, \mathbb{P}\right)$ is the probability space on which the white noise $W=(W(f))_{f \in L^{2}\left(\mathbb{R}^{d}\right)}$ is defined, for every $\boldsymbol{\nu} \in L^{2}\left(\mathbb{R}^{d}\right)$ we introduce a new probability $\mathbb{P}_{\boldsymbol{\nu}}$ on $\Omega_{W}$ by

$$
\frac{\mathrm{d} \mathbb{P}_{\boldsymbol{\nu}}}{\mathrm{d} \mathbb{P}}:=e^{W(\boldsymbol{\nu})-\frac{1}{2} \mathbb{E}\left[W(\boldsymbol{\nu})^{2}\right]}=e^{\int_{\mathbb{R}^{d}} \boldsymbol{\nu}(x) W(\mathrm{~d} x)-\frac{1}{2} \int_{\mathbb{R}^{d}} \boldsymbol{\nu}(x)^{2} \mathrm{~d} x} .
$$

It turns out that the "biased stochastic integrals" in (2.13) have the same joint distribution as the ordinary stochastic integrals (with $\boldsymbol{\nu}$ replaced by 0 ) under the probability $\mathbb{P}_{\boldsymbol{\nu}}$, by the Cameron-Martin theorem (cf. Appendix $\mathrm{A}$ ). As a consequence, the random variable $\boldsymbol{\Psi}_{0}$ in (2.12) enjoys the following equality in distribution, setting $\boldsymbol{\nu}(x):=\boldsymbol{\mu}_{0}(x) / \boldsymbol{\sigma}_{0}$ :

$$
\boldsymbol{\Psi}_{0} \stackrel{d}{=} \sum_{k=0}^{\infty} \frac{1}{k !} \int \cdots \int_{\left(\mathbb{R}^{d}\right)^{k}} \boldsymbol{\psi}_{0}\left(y_{1}, \ldots, y_{k}\right) \boldsymbol{\sigma}_{0}^{k} W\left(\mathrm{~d} y_{1}\right) \cdots W\left(\mathrm{~d} y_{k}\right) \quad \text { under } \mathbb{P}_{\boldsymbol{\nu}},
$$

provided the series (either in (2.12) or (2.16), equivalently) converges in probability.

Let us now assume the weaker version of relation (2.14) for $\varepsilon=0$, i.e.

$$
\sum_{k=0}^{\infty} \frac{1}{k !}\left(\sigma_{0}^{2}\right)^{k}\left\|\psi_{0}\right\|_{L^{2}\left(\left(\mathbb{R}^{d}\right)^{k}\right)}<\infty
$$


Under this condition, the series in (2.16) converges in $L^{2}$ under the original probability $\mathbb{P}$, by the Itô isometry (2.2). Since the Radon-Nikodym density (2.15) has finite moments of all orders, it follows by (2.16) and an application of Hölder inequality (see (5.11) for the details) that the series in (2.12) defining $\boldsymbol{\Psi}_{0}$ converges in $L^{p}$ for every $p \in(0,2)$ when (2.17) holds (even though it might not converge in $L^{2}$, if (2.14) fails).

As a consequence, by performing an $L^{p}$ analysis for $p<2$, we can weaken condition (iii) in Theorem 2.3, setting $\varepsilon=0$ in (2.11), under mild restrictions on the disorder distribution (due to the implementation of a change of measure like in (2.16) for polynomial chaos).

Theorem 2.5. (Convergence of polynomial chaos to Wiener chaos, $L^{2-}$ case) Let the same assumptions as in Theorem 2.3 hold, with condition (iii) therein weakened by setting $\varepsilon=0$ in (2.11). Assume further that $\lim _{\delta \downarrow 0}\left\|\mu_{\delta}\right\|_{\infty}=0$, and that either of the following two conditions is satisfied:

(a) $\inf _{\delta \in(0,1), x \in \mathbb{T}_{\delta}} \min \left\{\mathbb{P}\left(\zeta_{\delta, x}>0\right), \mathbb{P}\left(\zeta_{\delta, x}<0\right), \operatorname{Var}\left(\zeta_{\delta, x} \mid \zeta_{\delta, x}>0\right), \operatorname{Var}\left(\zeta_{\delta, x} \mid \zeta_{\delta, x}<0\right)\right\}>0 ;$

(b)

$$
\forall C>0: \quad \lim _{\delta \downarrow} \sum_{I \in \mathcal{P}_{\text {fin }}\left(\mathbb{T}_{\delta}\right),|I|>\left\|\mu_{\delta}\right\|_{\infty}^{-1}} e^{C\left\|\mu_{\delta}\right\|_{\infty}|I|}\left(\sigma_{\delta}^{2}\right)^{|I|} \psi_{\delta}(I)^{2}=0 .
$$

Then the polynomial chaos expansion $\Psi_{\delta}\left(\zeta_{\delta}\right)$ is well-defined and converges in distribution as $\delta \downarrow 0$ to the random variable $\boldsymbol{\Psi}_{0}$ defined by (2.12), or equivalently (2.16). For all $p \in(0,2)$, the series therein converges in $L^{p}$, and furthermore $\mathbb{E}\left[\left|\Psi_{\delta}\right|^{p}\right] \rightarrow \mathbb{E}\left[\left|\Psi_{0}\right|^{p}\right]$. The conclusion extends to a finite collection $\left(\Psi_{i, \delta}\left(\zeta_{\delta}\right)\right)_{1 \leq i \leq M}$.

2.4. Lindeberg PRINCIPle For polynomial CHaOs. The key ingredients in our proof of Theorem 2.3 are two Lindeberg principles for polynomial chaos. As we discuss in Remark 2.7, they extend Theorem 3.18 in [MOO10] in two ways: firstly, we relax the finite third moment assumption of MOO10 to an optimal condition of uniform integrability of the square of the random variables; secondly, we allow random variables with non-zero mean.

We point out that the first extension is actually not needed for our applications to disordered systems, due to the assumption of finite exponential moments for the disorder random variables. However, it is an extension of general interest and will be useful if one attempts to weaken the moment assumptions on the disorder random variables, as discussed at the end of Section 1.3. We remark that Lindeberg principles have also played crucial roles in recent breakthrough results on random matrices [C06, TV11].

Given a polynomial chaos expansion $\Psi(\zeta)$ with respect to a family $\zeta:=\left(\zeta_{i}\right)_{i \in \mathbb{T}}$ of independent random variables (cf. Subsection 2.2), we will control how the distribution of $\Psi(\zeta)$ changes when we replace $\zeta$ by independent Gaussian random variables $\xi:=\left(\xi_{i}\right)_{i \in \mathbb{T}}$ with the same mean and variance as $\zeta$.

Given a multi-linear polynomial $\Psi(x)=\Psi\left(\left(x_{i}\right)_{i \in \mathbb{T}}\right)$ as in (2.5), with kernel $\psi$, we set

$$
\mathrm{C}_{\Psi}:=\sum_{I \in \mathcal{P} \text { fin }(\mathbb{T}), I \neq \emptyset} \psi(I)^{2}
$$

and define the influence of the $i$-th variable $x_{i}$ on $\Psi$ by

$$
\operatorname{Inf}_{i}[\Psi]:=\sum_{I \in \mathcal{P}^{\mathrm{fin}}(\mathbb{T}), I \ni i} \psi(I)^{2} .
$$


Note that, if $\zeta=\left(\zeta_{i}\right)_{i \in \mathbb{T}}$ are independent random variables with zero mean and unit variance,

$$
C_{\Psi}=\operatorname{Var}[\Psi(\zeta)], \quad \operatorname{Inf}_{i}[\Psi]=\mathbb{E}\left[\operatorname{Var}\left[\Psi(\zeta) \mid\left(\zeta_{j}\right)_{j \in \mathbb{T} \backslash\{i\}}\right]\right],
$$

which is just the influence of the random variable $\zeta_{j}$ on $\Psi(\zeta)$ introduced in MOO10] (for more on the notion of influence, see e.g. [KKL88, BKKKL92] and the references in [MOO10]). We also define the degree $\ell$ truncations $\Psi^{\leq \ell}$ and $\Psi^{>\ell}$ of the multi-linear polynomial $\Psi$ by

$$
\Psi^{\leq \ell}(x):=\sum_{I \in \mathcal{P}^{\operatorname{fin}}(\mathbb{T}),|I| \leq \ell} \psi(I) x^{I}, \quad \Psi^{>\ell}(x):=\sum_{I \in \mathcal{P}^{\mathrm{fin}}(\mathbb{T}),|I|>\ell} \psi(I) x^{I},
$$

whose kernels will be denoted by $\psi^{\leq \ell}(I)=\psi(I) \mathbb{1}_{\{|I| \leq \ell\}}$ and $\psi^{>\ell}(I)=\psi(I) \mathbb{1}_{\{|I|>\ell\}}$.

We are now ready to state and comment on our Lindeberg principles, that will be proved in Section 4 .

Theorem 2.6 (Lindeberg principle, zero mean case). Let $\zeta=\left(\zeta_{i}\right)_{i \in \mathbb{T}}$ and $\xi=\left(\xi_{i}\right)_{i \in \mathbb{T}}$ be two families of independent random variables, with zero mean and unit variance. Let $\Psi(x)$ be a multi-linear polynomial as in (2.5), with $\mathrm{C}_{\Psi}=\sum_{I \in \mathcal{P} \text { fin }(\mathbb{T})} \psi(I)^{2}<\infty$. Then the polynomial chaos expansions $\Psi(\zeta), \Psi(\xi)$ are well-defined $L^{2}$ random variables.

Defining for $M \in[0, \infty]$ the maximal truncated moments

$$
m_{2}^{>M}:=\sup _{X \in \bigcup_{i \in \mathbb{T}}\left\{\zeta_{i}, \xi_{i}\right\}} \mathbb{E}\left[X^{2} \mathbb{1}_{|X|>M}\right], \quad m_{3}^{\leq M}:=\sup _{X \in \bigcup_{i \in \mathbb{T}}\left\{\zeta_{i}, \xi_{i}\right\}} \mathbb{E}\left[|X|^{3} \mathbb{1}_{|X| \leq M}\right],
$$

the following relation holds: for every $f: \mathbb{R} \rightarrow \mathbb{R}$ of class $\mathscr{C}^{3}$ with

$$
C_{f}:=\max \left\{\left\|f^{\prime}\right\|_{\infty},\left\|f^{\prime \prime}\right\|_{\infty},\left\|f^{\prime \prime \prime}\right\|_{\infty}\right\}<\infty,
$$

for every $\ell \in \mathbb{N}$, and for every $M \in(0, \infty]$ large enough such that $m_{2}^{>M} \leq \frac{1}{4}$, one has

$$
\begin{aligned}
|\mathbb{E}[f(\Psi(\zeta))]-\mathbb{E}[f(\Psi(\xi))]| \leq C_{f}\{ & 2 \sqrt{C_{\Psi>\ell}}+C_{\Psi \leq \ell} 16 \ell^{2} m_{2}^{>M} \\
& \left.+C_{\Psi \leq \ell} 70^{\ell+1}\left(m_{3}^{\leq M}\right)^{\ell} \sqrt{\max _{i \in \mathbb{T}}\left(\operatorname{Inf}_{i}[\Psi \leq \ell]\right)}\right\},
\end{aligned}
$$

where C., $\operatorname{Inf}_{i}[\cdot]$ and $\Psi \leq \ell, \Psi^{>\ell}$ are defined in (2.19), (2.20) and (2.21).

Intuitively, this theorem shows that $\Psi(\zeta)$ and $\Psi(\xi)$ are close in distribution when the right hand side of (2.24) is small. Despite its technical appearance, each of the three terms inside the brackets can be easily controlled:

- The first term is controlled by $C_{\Psi>\ell}=\sum_{|I|>\ell} \psi(I)^{2}$, which is small for $\ell$ large.

- The second term is controlled by $m_{2}^{>M}$, which is small for $M$ large if the random variables $\left(\zeta_{i}\right)_{i \in \mathbb{T}}$ and $\left(\xi_{i}\right)_{i \in \mathbb{T}}$ have uniformly integrable squares (e.g., if they are i.i.d.).

- The third term is controlled by the maximal influence $\max _{i \in \mathbb{T}} \operatorname{Inf}_{i}[\Psi \leq \ell]$, which is small if the multi-linear polynomial $\Psi^{\leq \ell}$ is sufficiently "spread-out".

In particular, we shall see that the conditions of Theorem 2.3 allow us to exploit (2.24).

Remark 2.7. When the polynomial $\Psi=\Psi^{\leq \ell}$ has degree $\ell$ and the random variables $\zeta_{i}, \xi_{i}$ have third absolute moments bounded by $m_{3}<\infty$, relation (2.24) for $M=\infty$ reduces to

$$
|\mathbb{E}[f(\Psi(\zeta))]-\mathbb{E}[f(\Psi(\xi))]| \leq C_{f} C_{\Psi} 70^{\ell+1}\left(m_{3}\right)^{\ell} \max _{i \in \mathbb{T}} \sqrt{\operatorname{Inf}_{i}[\Psi]}
$$


This is the key estimate proved by Mossel, O'Donnell and Oleszkiewicz in [MOO10, see Theorem 3.18 under hypothesis $\boldsymbol{H 2}$, with the prefactor $70^{\ell+1}$ instead of $30^{\ell}$. Our Theorem [2.6 thus provides an extension of [MOO10, Theorem 3.18] to finite second-moment assumptions.

Some of the results in [MOO10] are formulated in the more general setting of multi-linear polynomials over orthornormal ensembles. Although we stick for simplicity to the case of independent random variables, our approach can be adapted to deal with orthonormal ensembles. In fact, we follow the same line of proof of MOO10, which is based on Lindeberg's original approach, with two refinements: a sharper approximation of the remainder in Taylor's expansion and a fine truncation on the random variables, cf. Section 4 for details.

As a corollary to Theorem 2.6, we can treat the case where we add non-zero mean to the random variables $\left(\zeta_{i}\right)_{i \in \mathbb{T}}$ and $\left(\xi_{i}\right)_{i \in \mathbb{T}}$. The following result is also proved in Section 4 .

Theorem 2.8 (Lindeberg principle, non-zero mean case). Let $\zeta=\left(\zeta_{i}\right)_{i \in \mathbb{T}}$ and $\xi=$ $\left(\xi_{i}\right)_{i \in \mathbb{T}}$ be as in Theorem [2.6, and define the maximal truncated moments $m_{2}^{>M}, m_{3}^{\leq M}$ by (2.22). Let $\mu:=\left(\mu_{i}\right)_{i \in \mathbb{T}}$ be a family of real numbers with

$$
\mathrm{c}_{\mu}:=\sum_{i \in \mathbb{T}} \mu_{i}^{2}<\infty
$$

and define the $\mu$-biased families $\widetilde{\zeta}:=\zeta+\mu=\left(\zeta_{i}+\mu_{i}\right)_{i \in \mathbb{T}}$ and $\widetilde{\xi}:=\xi+\mu=\left(\xi_{i}+\mu_{i}\right)_{i \in \mathbb{T}}$.

Let $\Psi(x)$ be a multi-linear polynomial as in (2.5). Setting for $\varepsilon>0$

$$
\Psi^{(\varepsilon)}(x)=\sum_{I \in \mathcal{P}^{\text {fin }}(\mathbb{T})}(1+\varepsilon)^{|I| / 2} \psi(I) x^{I}
$$

assume that $\mathrm{C}_{\Psi^{(\varepsilon)}}=\sum_{I \in \mathcal{P}^{\text {fin }}(\mathbb{T})}(1+\varepsilon)^{|I|} \psi(I)^{2}<\infty$ for some $\varepsilon>0$. Then the polynomial chaos expansions $\Psi(\widetilde{\zeta})$ and $\Psi(\widetilde{\xi})$ are well-defined $L^{2}$ random variables.

For every $f: \mathbb{R} \rightarrow \mathbb{R}$ of class $\mathscr{C}^{3}$ with $C_{f}<\infty$, cf. (2.23), for every $\ell \in \mathbb{N}$ and for every $M \in[0, \infty]$ large enough such that $m_{2}^{>M} \leq \frac{1}{4}$, the following relation holds:

$$
\begin{aligned}
|\mathbb{E}[f(\Psi(\widetilde{\zeta}))]-\mathbb{E}[f(\Psi(\widetilde{\xi}))]| \leq & e^{2 c_{\mu} / \varepsilon} C_{f}\left\{2 \sqrt{C_{\Psi^{(\varepsilon),>\ell}}}+C_{\Psi^{(\varepsilon), \leq \ell}} 16 \ell^{2} m_{2}^{>M}\right. \\
& \left.+C_{\Psi^{(\varepsilon),}, \leq \ell} 70^{\ell+1}\left(m_{3}^{\leq M}\right)^{\ell} \sqrt{\max _{i \in \mathbb{T}}\left(\operatorname{Inf}_{i}\left[\Psi^{(\varepsilon), \leq \ell}\right]\right)}\right\}
\end{aligned}
$$

where C., $\operatorname{Inf}_{i}[\cdot]$ are defined in (2.19), (2.20) and $\Psi^{(\varepsilon),>\ell}, \Psi^{(\varepsilon), \leq \ell}$ are defined as in (2.21).

\section{SCALING LIMITS OF DISORDERED SYSTEMS}

In this section, which can be read independently of Sections 1 and 2 , we consider three much-studied statistical mechanics models: the disordered pinning model (Subsection 3.1), the (long-range) directed polymer model in dimension $1+1$ (Subsection 3.2), and the twodimensional random field Ising model (Subsection 3.3). For each model, we show that the partition function has a non-trivial limit in distribution, in the continuum and weak disorder regime, given by an explicit Wiener chaos expansion with respect to the white noise on $\mathbb{R}^{d}$ (see Subsection 2.1 for some reminders). The proofs, given in Sections 6, 7 and 8, are based on the general convergence results of Section 2 (cf. Theorem 2.3). 
For each model, the disorder will be given by a countable family of i.i.d. random variables $\omega_{i}$ with zero mean, finite variance and locally finite exponential moments:

$$
\mathbb{E}\left[\omega_{i}\right]=0, \quad \operatorname{Var}\left(\omega_{i}\right)=1, \quad \exists t_{0}>0: \quad \Lambda(t):=\log \mathbb{E}\left[e^{t \omega_{i}}\right]<\infty \text { for }|t|<t_{0} .
$$

Our approach actually works in the much more general setting when disorder is given by a triangular array of independent (but not necessarily identically distributed) random variables, in the spirit of Theorem 2.3. but we stick to the i.i.d. case for the sake of simplicity.

3.1. Disordered PINNING MODel. Consider a discrete renewal process $\tau:=\left\{\tau_{n}\right\}_{n \geq 0}$, that is $\tau_{0}=0$ and the increments $\left\{\tau_{n}-\tau_{n-1}\right\}_{n \geq 1}$ are i.i.d. $\mathbb{N}$-valued random variables. We assume that $\tau$ is non-terminating, that is $\mathrm{P}\left(\tau_{1}<\infty\right)=1$, and that

$$
\mathrm{P}\left(\tau_{1}=n\right)=\frac{L(n)}{n^{1+\alpha}}, \quad \forall n \in \mathbb{N},
$$

where $\alpha \in[0,+\infty)$ and $L:(0, \infty) \rightarrow(0, \infty)$ is a slowly varying function [BGT87. One could also consider the periodic case, when (3.2) holds for $n \in p \mathbb{N}$ and $\mathrm{P}\left(\tau_{1}=n\right)=0$ if $n \notin p \mathbb{N}$, for some period $p \in \mathbb{N}$. For simplicity, we focus on the aperiodic case $p=1$.

Let $\omega=\left(\omega_{n}\right)_{n \in \mathbb{N}_{0}}$ be a sequence of i.i.d. random variables, independent of $\tau$, satisfying (3.1). The disordered pinning model is the random probability law $\mathrm{P}_{N, \beta, h}^{\omega}$ on subsets on $\mathbb{N}_{0}$, indexed by $\omega$ and by $N \in \mathbb{N}, \beta \geq 0$ and $h \in \mathbb{R}$, defined by

$$
\mathrm{dP}_{N, \beta, h}^{\omega}(\tau):=\frac{1}{Z_{N, \beta, h}^{\omega}} e^{\sum_{n=1}^{N}\left(\beta \omega_{n}-\Lambda(\beta)+h\right) \mathbb{1}_{\{n \in \tau\}}} \mathrm{dP}(\tau)
$$

where we recall that $\Lambda(\beta):=\log \mathbb{E}\left[e^{\beta \omega_{1}}\right]$, and the partition function $Z_{N, \beta, h}^{\omega}$ is defined by

$$
Z_{N, \beta, h}^{\omega}:=\mathrm{E}\left[e^{\sum_{n=1}^{N}\left(\beta \omega_{n}-\Lambda(\beta)+h\right) \mathbb{1}_{\{n \in \tau\}}}\right] .
$$

We also consider the conditioned partition function

$$
Z_{N, \beta, h}^{\omega, c}:=\mathrm{E}\left[e^{\sum_{n=1}^{N}\left(\beta \omega_{n}-\Lambda(\beta)+h\right) \mathbb{1}_{\{n \in \tau\}}} \mid N \in \tau\right] .
$$

The disordered pinning model exhibits an interesting localization/delocalization phase transition. This can be quantified via the (quenched) free energy, which is defined as

$$
F(\beta, h):=\lim _{N \rightarrow \infty} \frac{1}{N} \log Z_{N, \beta, h}^{\omega}=\lim _{N \rightarrow \infty} \frac{1}{N} \mathbb{E}\left[\log Z_{N, \beta, h}^{\omega}\right], \quad \mathbb{P}(\mathrm{d} \omega) \text {-a.s. }
$$

By restricting the partition function to configurations such that $\tau \cap[1, N]=\emptyset$, it is easily seen that $F(\beta, h) \geq 0$. The localized and delocalized regimes $(\mathcal{L}, \mathcal{D}$ respectively) can be defined as

$$
\mathcal{L}:=\{(\beta, h): F(\beta, h)>0\}, \quad \mathcal{D}:=\{(\beta, h): F(\beta, h)=0\} .
$$

We refer to [G10] for more information on the structure of the phase transition and, in particular, for quantitative estimates on the critical curve

$$
h_{c}(\beta):=\sup \{h \in \mathbb{R}: F(\beta, h)=0\}=\inf \{h \in \mathbb{R}: F(\beta, h)>0\} .
$$

We can now state our main result on the disordered pinning model, to be proved in Section 6. To lighten notation, we write $Z_{N t, \beta, h}^{\omega}$ to mean $Z_{\lfloor N t\rfloor, \beta, h}^{\omega}$. 
Theorem 3.1 (Scaling limit of disordered pinning models). Let the aperiodic renewal process $\tau$ either satisfy (3.2) for some $\alpha \in\left(\frac{1}{2}, 1\right)$, or have finite mean $\mathrm{E}\left[\tau_{1}\right]<\infty$ (which happens, in particular, when (3.2) holds with $\alpha>1$ ). For $N \in \mathbb{N}, \hat{\beta}>0$ and $\hat{h} \in \mathbb{R}$, set

$$
\beta_{N}=\left\{\begin{array}{ll}
\hat{\beta} \frac{L(N)}{N^{\alpha-1 / 2}} & \text { if } \frac{1}{2}<\alpha<1 \\
\hat{\beta} \frac{1}{\sqrt{N}} & \text { if } \mathrm{E}\left[\tau_{1}\right]<\infty
\end{array}, \quad h_{N}=\left\{\begin{array}{l}
\hat{h} \frac{L(N)}{N^{\alpha}} \quad \text { if } \frac{1}{2}<\alpha<1 \\
\hat{h} \frac{1}{N} \quad \text { if } \mathrm{E}\left[\tau_{1}\right]<\infty
\end{array} .\right.\right.
$$

Then, for every $t \geq 0$, the conditioned partition function $Z_{N t, \beta_{N}, h_{N}}^{\omega, c}$ of the disordered pinning model converges in distribution as $N \rightarrow \infty$ to the random variable $Z_{t, \hat{\beta}, \hat{h}}^{W, c}$ given by

$$
Z_{t, \hat{\beta}, \hat{h}}^{W, c}:=1+\sum_{k=1}^{\infty} \frac{1}{k !} \int \ldots \int \boldsymbol{\psi}_{t}^{c}\left(t_{1}, \ldots, t_{k}\right) \prod_{i=1}^{k}\left(\hat{\beta} W\left(\mathrm{~d} t_{i}\right)+\hat{h} \mathrm{~d} t_{i}\right),
$$

where $W(\cdot)$ denotes white noise on $\mathbb{R}$ and $\boldsymbol{\psi}_{t}^{c}\left(t_{1}, \ldots, t_{k}\right)$ is a symmetric function, defined for $0<t_{1}<\cdots<t_{k}<t$ by

$$
\psi_{t}^{c}\left(t_{1}, \ldots, t_{k}\right)=\left\{\begin{array}{ll}
\frac{C_{\alpha}^{k} t^{1-\alpha}}{t_{1}^{1-\alpha}\left(t_{2}-t_{1}\right)^{1-\alpha} \cdots\left(t_{k}-t_{k-1}\right)^{1-\alpha}\left(t-t_{k}\right)^{1-\alpha}} & \text { if } \frac{1}{2}<\alpha<1 \\
\frac{1}{\mathrm{E}\left[\tau_{1}\right]^{k}} & \text { if } \mathrm{E}\left[\tau_{1}\right]<\infty
\end{array},\right.
$$

where $C_{\alpha}:=\frac{\alpha \sin (\pi \alpha)}{\pi}$. The series in (3.8) converges in $L^{2}$, and one has the convergence of the corresponding second moments: $\mathbb{E}\left[\left(Z_{N t, \beta_{N}, h_{N}}^{\omega, c}\right)^{2}\right] \rightarrow \mathbb{E}\left[\left(Z_{t, \hat{\beta}, \hat{h}}^{W, c}\right)^{2}\right]$ as $N \rightarrow \infty$.

An analogous statement holds for the free (unconditioned) partition function $Z_{N t, \beta_{N}, h_{N}}^{\omega}$, where the limiting random variable $\boldsymbol{Z}_{t, \hat{\beta}, \hat{h}}^{W}$ is defined as in (3.8), with kernel

$$
\psi\left(t_{1}, \ldots, t_{k}\right):=\left\{\begin{array}{cc}
\frac{C_{\alpha}^{k}}{t_{1}^{1-\alpha}\left(t_{2}-t_{1}\right)^{1-\alpha} \cdots\left(t_{k}-t_{k-1}\right)^{1-\alpha}} & \text { if } \frac{1}{2}<\alpha<1 \\
\frac{1}{\mathrm{E}\left[\tau_{1}\right]^{k}} & \text { if } \mathrm{E}\left[\tau_{1}\right]<\infty
\end{array} .\right.
$$

When $\mathrm{E}\left[\tau_{1}\right]<\infty$, for both the free and conditioned case, the continuum partition function has an explicit distribution: for every $t \geq 0$

$$
Z_{t, \hat{\beta}, \hat{h}}^{W, c} \stackrel{d}{=} Z_{t, \hat{\beta}, \hat{h}}^{W} \stackrel{d}{=} \exp \left\{\frac{\hat{\beta}}{\mathrm{E}\left[\tau_{1}\right]} W_{t}+\left(\frac{\hat{h}}{\mathrm{E}\left[\tau_{1}\right]}-\frac{\hat{\beta}^{2}}{2 \mathrm{E}\left[\tau_{1}\right]^{2}}\right) t\right\},
$$

where $W=\left(W_{t}\right)_{t \geq 0}$ denotes a standard Brownian motion.

Remark 3.2. The stochastic integrals in (3.8) can be rewritten more directly as follows: denoting by $W=\left(W_{t}\right)_{t \geq 0}$ a standard Brownian motion, we have

$$
Z_{t, \hat{\beta}, \hat{h}}^{W}:=1+\sum_{k=1}^{\infty} \int_{0<t_{1}<\cdots<t_{k}<t} \ldots \int_{t}\left(t_{1}, \ldots, t_{k}\right) \prod_{i=1}^{k}\left(\hat{\beta} \mathrm{d} W_{t_{i}}+\hat{h} \mathrm{~d} t_{i}\right),
$$

where the integrals can be viewed as ordinary Ito integrals: it suffices to first integrate over $\left(\hat{\beta} \mathrm{d} W_{t_{1}}+\hat{h} \mathrm{~d} t_{1}\right)$ for $t_{1} \in\left(0, t_{2}\right)$, then over $\left(\hat{\beta} \mathrm{d} W_{t_{2}}+\hat{h} \mathrm{~d} t_{2}\right)$ for $t_{2} \in\left(0, t_{3}\right)$, etc. 
Remark 3.3. Theorem 3.1 extends readily to the convergence of the joint distribution of a finite collection of partition functions (conditioned or free). Analogously, the two parameter family of partition functions

$$
Z_{\beta_{N}, h_{N}}^{\omega, c}(N s, N t):=\mathrm{E}\left[e^{\sum_{n=N s+1}^{N t-1}\left(\beta \omega_{n}-\Lambda(\beta)+h\right) \mathbb{1}_{\{n \in \tau\}}} \mid N s, N t \in \tau\right], \quad \text { for } 0<s<t,
$$

converges in finite-dimensional distributions to a two-parameter process $\left(\boldsymbol{Z}_{\hat{\beta}, \hat{h}}^{W, c}(s, t)\right)_{0<s<t}$. In [CSZ14] we upgrade this result to a convergence in distribution in the space of continuous functions, equipped with the uniform topology. This allows to construct the continuum limit of the disordered pinning measure $\mathrm{P}_{N, \beta, h}^{\omega}$.

Remark 3.4. It is natural to call the random variable $\boldsymbol{Z}_{t, \hat{\beta}, \hat{h}}^{W}$ in Theorem 3.1 the continuum partition function and to define the corresponding continuum free energy

$$
\boldsymbol{F}^{(\alpha)}(\hat{\beta}, \hat{h})=\lim _{t \rightarrow \infty} \frac{1}{t} \mathbb{E}\left[\log \boldsymbol{Z}_{t, \hat{\beta}, \hat{h}}^{W}\right]
$$

where $\mathbb{E}$ denotes expectation with respect to the white noise $W$, provided the limit exists. For $\frac{1}{2}<\alpha<1$, we expect that the continuum and discrete free energies are related via

$$
\boldsymbol{F}^{(\alpha)}(\hat{\beta}, \hat{h})=\lim _{\delta \downarrow 0} \frac{F\left(\delta^{\alpha-\frac{1}{2}} L\left(\frac{1}{\delta}\right) \hat{\beta}, \delta^{\alpha} L\left(\frac{1}{\delta}\right) \hat{h}\right)}{\delta} .
$$

This would follow if one could interchange the limits in the formal computation

$$
\boldsymbol{F}^{(\alpha)}(\hat{\beta}, \hat{h})=\lim _{t \rightarrow \infty} \frac{1}{t} \log Z_{t, \hat{\beta}, \hat{h}}^{W} \stackrel{d}{=} \lim _{t \rightarrow \infty} \frac{1}{t} \lim _{N \rightarrow \infty} \log Z_{t N, \beta_{N}, h_{N}}^{\omega} .
$$

where $\beta_{N}, h_{N}$ scale as in (3.7). Such an interchange of limits has been made possible in the related copolymer model, cf. [BdH97, CG10]. Proving the validity of relation (3.15) is a very interesting open problem. Even the existence of the continuum free energy in (3.14) - possibly also in the $\mathbb{P}$-a.s. sense, like for the discrete case (3.5) - is a non-trivial issue.

Relation (3.15) is appealing because of its implication of universality: it states that the discrete free energy $F(\beta, h)$ has a universal shape in the weak disorder regime $\beta, h \rightarrow 0$, given by the continuum free energy, which depends only on the parameter $\alpha$ and not on finer details of the renewal distribution. Inverting the relation $\beta=\delta^{\alpha-\frac{1}{2}} L\left(\frac{1}{\delta}\right)$, it is possible to rewrite (3.15) for $\hat{\beta}=1$ as

$$
\boldsymbol{F}^{(\alpha)}(1, \hat{h})=\lim _{\beta \downarrow 0} \frac{F\left(\beta, \widetilde{L}\left(\frac{1}{\beta}\right) \beta^{\frac{2 \alpha}{2 \alpha-1}} \hat{h}\right)}{\widehat{L}\left(\frac{1}{\beta}\right) \beta^{\frac{2}{2 \alpha-1}}},
$$

where $\widetilde{L}(\cdot)$ and $\widehat{L}(\cdot)$ are suitable slowly varying functions determined by $L(\cdot)$. by

Given a slowly varying function $\phi$ and $\gamma>0$, we define the slowly varying functions $\bar{\phi}_{\gamma}$ and $\phi^{*}$

$$
\bar{\phi}_{\gamma}(x):=\frac{1}{\phi\left(x^{1 / \gamma}\right)}, \quad \phi^{*}(x \phi(x)) \sim \frac{1}{\phi(x)} \quad \text { as } x \rightarrow \infty,
$$

where the existence of $\phi^{*}$ is guaranteed by [BGT87, Theorems 1.5.13]. Then as $\beta \downarrow 0$

$$
\delta^{\alpha-\frac{1}{2}} L\left(\frac{1}{\delta}\right)=\beta \quad \Longrightarrow \quad \frac{1}{\delta^{\alpha-\frac{1}{2}}} \bar{L}_{\alpha-\frac{1}{2}}\left(\frac{1}{\delta^{\alpha-\frac{1}{2}}}\right) \sim \frac{1}{\beta} \quad \Longrightarrow \quad L\left(\frac{1}{\delta}\right) \sim\left(\bar{L}_{\alpha-\frac{1}{2}}\right)^{*}\left(\frac{1}{\beta}\right)
$$


hence by $\delta^{\alpha-\frac{1}{2}} L\left(\frac{1}{\delta}\right)=\beta$ we obtain $\delta \sim \beta^{\frac{2}{2 \alpha-1}} \widehat{L}\left(\frac{1}{\beta}\right)$ and $\delta^{\alpha} L\left(\frac{1}{\delta}\right) \sim \beta^{\frac{2 \alpha}{2 \alpha-1}} \widetilde{L}\left(\frac{1}{\beta}\right)$, where

$$
\widehat{L}(x):=\left[\left(\bar{L}_{\alpha-\frac{1}{2}}\right)^{*}(x)\right]^{-\frac{2}{2 \alpha-1}}, \quad \widetilde{L}(x):=\left[\left(\bar{L}_{\alpha-\frac{1}{2}}\right)^{*}(x)\right]^{-\frac{1}{2 \alpha-1}} .
$$

Plugging this into (3.15) (with $\hat{\beta}=1$ ), we get (3.17).

Defining the critical curve of the continuum free energy in analogy with (3.6), i.e.

$$
\boldsymbol{h}_{c}^{(\alpha)}(\hat{\beta}):=\sup \left\{\hat{h} \in \mathbb{R}: \boldsymbol{F}^{(\alpha)}(\hat{\beta}, \hat{h})=0\right\}=\inf \left\{\hat{h} \in \mathbb{R}: \boldsymbol{F}^{(\alpha)}(\hat{\beta}, \hat{h})>0\right\}
$$

relation (3.17) leads us to the following

Conjecture 3.5. For any disordered pinning model satisfying (3.2) with $\alpha \in\left(\frac{1}{2}, 1\right)$, the critical curve $h_{c}(\beta)$ has the following universal asymptotic behavior (defining $\widetilde{L}(\cdot)$ by (3.18)):

$$
\lim _{\beta \downarrow 0} \frac{h_{c}(\beta)}{\widetilde{L}\left(\frac{1}{\beta}\right) \beta^{\frac{2 \alpha}{2 \alpha-1}}}=\boldsymbol{h}_{c}^{(\alpha)}(1) .
$$

Further support to this conjecture is provided by the fact that (non-matching) upper and lower bounds for $h_{c}(\beta)$ of the order $\widetilde{L}\left(\frac{1}{\beta}\right) \beta^{\frac{2 \alpha}{2 \alpha-1}}$ were proved in [A08, AZ09].

Remark 3.6. The case when (3.2) holds with $\alpha=1$ and $\mathrm{E}\left[\tau_{1}\right]=\infty$, i.e. $\sum_{n \in \mathbb{N}} L(n) / n=\infty$, can also be included in Theorem 3.1 (we have omitted it for notational lightness), setting

$$
\beta_{N}=\hat{\beta} \frac{\ell(N)}{\sqrt{N}}, \quad h_{N}=\hat{h} \frac{\ell(N)}{N}, \quad \text { where } \quad \ell(N):=\sum_{n=1}^{N} \frac{L(n)}{n}
$$

is a slowly varying function, and with $\boldsymbol{\psi}_{t}^{c}\left(t_{1}, \ldots, t_{k}\right)=\boldsymbol{\psi}\left(t_{1}, \ldots, t_{k}\right) \equiv 1$. This is easily checked from the proof in Section 6, because $\mathrm{P}(n \in \tau) \sim \frac{1}{\ell(n)}$, cf. [BGT87, Theorem 8.7.5].

On the other hand, the case $\alpha=1 / 2$ appears to be fundamentally different, because the continuum kernels $\boldsymbol{\psi}_{t}^{c}, \boldsymbol{\psi}$ are no longer $L^{2}$ integrable and therefore the stochastic integrals are not properly defined. When $\alpha=1 / 2$ and $\sum_{n \in \mathbb{N}} 1 /\left(n L(n)^{2}\right)=\infty$-in particular, when $L(n) \sim$ (const.) as $n \rightarrow \infty$, as for the simple random walk on $\mathbb{Z}$ - we expect that a nontrivial continuum limit should exist. This appears to be a challenging open problem.

3.2. Directed polymer model. Consider a random walk $S=\left(S_{n}\right)_{n \in \mathbb{N}_{0}}$ on $\mathbb{Z}$, with law P. Let $\omega=(\omega(n, x))_{n \in \mathbb{N}, x \in \mathbb{Z}}$ be a family of i.i.d. random variables, independent of $S$, with zero mean, unit variance and locally finite exponential moments, cf. (3.1). The $(1+1)$-dimensional directed polymer model is the random probability law $\mathrm{P}_{N, \beta}^{\omega}$ for the walk $S$ defined for $N \in \mathbb{N}$ and $\beta \geq 0$ by

$$
\mathrm{dP}_{N, \beta}^{\omega}(S):=\frac{1}{Z_{N, \beta}^{\omega}} e^{\sum_{n=1}^{N}\left(\beta \omega\left(n, S_{n}\right)-\Lambda(\beta)\right)} \mathrm{dP}(S),
$$

where we recall that $\Lambda(\beta):=\log \mathbb{E}\left[e^{\beta \omega_{n, x}}\right]$ and the partition function $Z_{N, \beta}^{\omega}$ is defined by

$$
Z_{N, \beta}^{\omega}=\mathrm{E}\left[e^{\beta \sum_{n=1}^{N} \omega\left(n, S_{n}\right)}\right] e^{-\Lambda(\beta) N} .
$$

For $y \in \mathbb{Z}$, we also define the constrained point-to-point partition function $Z_{N, \beta}^{\omega}(y)$ and the conditioned point-to-point partition function $Z_{N, \beta}^{\omega, c}(y)$, setting

$$
\begin{aligned}
& Z_{N, \beta}^{\omega}(y)=\mathrm{E}\left[e^{\beta \sum_{n=1}^{N} \omega\left(n, S_{n}\right)} \mathbb{1}_{\left\{S_{N}=y\right\}}\right] e^{-\Lambda(\beta) N}, \\
& Z_{N, \beta}^{\omega, c}(y)=\mathrm{E}\left[e^{\beta \sum_{n=1}^{N} \omega\left(n, S_{n}\right)} \mid S_{N}=y\right] e^{-\Lambda(\beta) N} .
\end{aligned}
$$


Plainly, $Z_{N, \beta}^{\omega}=\sum_{y \in \mathbb{Z}} Z_{N, \beta}^{\omega}(y)=\sum_{y \in \mathbb{Z}} Z_{N, \beta}^{\omega, c}(y) \mathrm{P}\left(S_{N}=y\right)$.

When $\left(S_{n}\right)_{n \geq 1}$ is the simple symmetric random walk on $\mathbb{Z}$, we have the much-studied $D i$ rected Polymer in Random Medium. First introduced in the physics literature in [HH85], this model has received particular attention due to its connection to the Kardar-Parisi-Zhang (KPZ) equation and its universality class, cf. CSY04, C12 for a review. In particular, the point-to-point partition function can be thought of as an approximation of the solution of the Stochastic Heat Equation (SHE), whose logarithm is the so-called Hopf-Cole solution of the KPZ equation. This was made rigorous in AKQ14a, showing that when $\beta=\beta_{N}$ is scaled as $N^{-1 / 4}$ (the so-called intermediate disorder regime) and $y$ is scaled as $N^{-1 / 2}$, the point-to-point partition function $Z_{N t, \beta}^{\omega}(y)$ converges in distribution to a continuum process, which solves the SHE.

Our approach allows to extend the results in AKQ14a. Not only can we deal with general zero mean, finite variance random walks, which are the natural "universality class" of the simple symmetric random walk on $\mathbb{Z}$; we can also consider random walks attracted to stable laws with index $\alpha \in(1,2)$, exploring new universality classes. When allowing "big jumps", it is natural to call $\mathrm{P}_{N, \beta}^{\omega}$ the long-range directed polymer model.

Let us now state precisely our assumptions on the random walk.

Assumption 3.7. Let $S=\left(S_{n}\right)_{n \geq 0}$ be a random walk on $\mathbb{Z}$, with $S_{0}=0$ and with i.i.d. increments $\left(S_{n}-S_{n-1}\right)_{n \geq 1}$, such that for some $\alpha \in(1,2]$ the following holds:

- $($ Case $\alpha=2): \mathrm{E}\left[S_{1}\right]=0$ and $\sigma^{2}:=\operatorname{Var}\left(S_{1}\right)<\infty$;

- (Case $1<\alpha<2): \mathrm{E}\left[S_{1}\right]=0$ and there exist $\gamma \in[-1,1]$ and $C \in(0, \infty)$ such that

$$
\mathrm{P}\left(S_{1}>n\right) \sim\left(C \frac{1+\gamma}{2}\right) \frac{1}{n^{\alpha}}, \quad \mathrm{P}\left(S_{1}<-n\right) \sim\left(C \frac{1-\gamma}{2}\right) \frac{1}{n^{\alpha}}, \quad \text { as } n \rightarrow \infty .
$$

This means that the random walk $S$ is in the domain of normal attraction of a stable law of index $\alpha \in(1,2]$ and (for $\alpha<2$ ) skewness parameter $\gamma$. (The adjective "normal" refers to the absence of slowly varying functions.) In other words, $S_{n} / n^{1 / \alpha}$ converges in distribution as $n \rightarrow \infty$ to a random variable $Y$, which has law $\mathcal{N}\left(0, \sigma^{2}\right)$ if $\alpha=2$, while for $1<\alpha<2$

$$
\mathrm{E}\left[e^{i t Y}\right]=e^{-c_{\alpha} C|t|^{\alpha}\left(1-i \gamma(\operatorname{sign} t) \tan \frac{\pi \alpha}{2}\right)}, \quad \text { for a suitable } \quad c_{\alpha}>0 .
$$

We remark that $Y$ satisfies the same conditions as $S_{1}$ in Assumption 3.7 and has an absolutely continuous law, with a bounded and continuous density $g(\cdot)$. For $t>0$ we set

$$
g_{t}(x):=\frac{1}{t^{1 / \alpha}} g\left(\frac{x}{t^{1 / \alpha}}\right) .
$$

We stress that $g(\cdot)$ depends only on the parameters $\left(\alpha, \sigma^{2}\right)$ or $(\alpha, \gamma, C)$ in Assumption 3.7

The period of a random walk $S$ on $\mathbb{Z}$ is the largest $p \in \mathbb{N}$ such that $\mathrm{P}\left(S_{1} \in p \mathbb{Z}+r\right)=1$, for some $r \in\{0, \ldots, p-1\}$. For instance, the simple symmetric random walk on $\mathbb{Z}$ has period $p=2$, because $\mathrm{P}\left(S_{1} \in 2 \mathbb{Z}+1\right)=1$. To lighten notation, given $(s, y) \in \mathbb{R}^{+} \times \mathbb{R}$, we write $Z_{s, \beta}^{\omega, c}(y)$ to mean $Z_{\widetilde{s}, \beta}^{\omega, c}(\widetilde{y})$, where $\widetilde{s}:=\lfloor s\rfloor$ and $\widetilde{y}:=\max \{z \in p \mathbb{Z}+r \widetilde{s}: z \leq y\}$.

We are ready to state our main result for this model, to be proved in Section 7 .

Theorem 3.8 (Scaling limits of directed polymer models). Let $S$ be a random walk on $\mathbb{Z}$ satisfying Assumption 3.7 for some $\alpha \in(1,2]$. For $N \in \mathbb{N}$ and $\hat{\beta}>0$, set

$$
\beta_{N}:=\frac{\hat{\beta}}{N^{\frac{\alpha-1}{2 \alpha}}} .
$$


For every $t \geq 0$ and $x \in \mathbb{R}$, the conditioned point-to-point partition function $Z_{N t, \beta_{N}}^{\omega, c}\left(N^{1 / \alpha} x\right)$ converges in distribution as $N \rightarrow \infty$ to the random variable $\boldsymbol{Z}_{t, \hat{\beta}}^{W, c}(x)$ given by

$$
\boldsymbol{Z}_{t, \hat{\beta}}^{W, c}(x):=1+\sum_{k=1}^{\infty} \frac{\hat{\beta}^{k}}{k !} \int_{([0, t] \times \mathbb{R})^{k}} \ldots \int_{t, x} \boldsymbol{\psi}_{t,}^{c}\left(\left(t_{1}, x_{1}\right), \ldots,\left(t_{k}, x_{k}\right)\right) \prod_{i=1}^{k} W\left(\mathrm{~d} t_{i} \mathrm{~d} x_{i}\right),
$$

where $W(\cdot)$ denotes space-time white noise (i.e., white noise on $\mathbb{R}^{2}$ ) and the symmetric function $\boldsymbol{\psi}_{t, x}^{c}\left(\left(t_{1}, x_{1}\right), \ldots,\left(t_{k}, x_{k}\right)\right)$ is defined for $0<t_{1}<\cdots<t_{k}<t$ by

$$
\boldsymbol{\psi}_{t, x}^{c}\left(\left(t_{1}, x_{1}\right), \ldots,\left(t_{k}, x_{k}\right)\right):=\left(\prod_{i=1}^{k} \sqrt{p} g_{t_{i}-t_{i-1}}\left(x_{i}-x_{i-1}\right)\right) \frac{g_{t-t_{k}}\left(x-x_{k}\right)}{g_{t}(x)}
$$

where $x_{0}:=0$ and $p \in \mathbb{N}$ is the period of the random walk. The series in (3.25) converges in $L^{2}$ and furthermore $\mathbb{E}\left[\left(Z_{N t, \beta_{N}}^{\omega, c}\left(N^{1 / \alpha} x\right)\right)^{2}\right] \rightarrow \mathbb{E}\left[\left(Z_{t, \hat{\beta}}^{W, c}(x)\right)^{2}\right]$ as $N \rightarrow \infty$.

An analogous statement holds for the free partition function $Z_{N t, \beta_{N}}^{\omega}$, where the limiting random variable $\boldsymbol{Z}_{t, \hat{\beta}}^{W}$ is defined as in (3.25), with kernel

$$
\psi_{t}\left(\left(t_{1}, x_{1}\right), \ldots,\left(t_{k}, x_{k}\right)\right):=\prod_{i=1}^{k} \sqrt{p} g_{t_{i}-t_{i-1}}\left(x_{i}-x_{i-1}\right) .
$$

Remark 3.9. Theorem 3.8 extends to the convergence of the joint distribution of a finite collection of partition functions (conditioned or free). In particular, the four-parameter family $Z_{N s, N t, \beta_{N}}^{\omega, c}\left(N^{1 / \alpha} x, N^{1 / \alpha} y\right)$, defined for $(s, x)$ and $(t, y)$ in $\mathbb{R}^{+} \times \mathbb{R}$ by

$$
Z_{N s, N t ; \beta_{N}}^{\omega, c}\left(N^{1 / \alpha} x, N^{1 / \alpha} y\right):=\mathrm{E}\left[e^{\sum_{n=N s+1}^{N t-1}\left(\beta \omega\left(n, S_{n}\right)-\Lambda(\beta)\right)} \mid S_{N s}=N^{1 / \alpha} x, S_{N t}=N^{1 / \alpha} y\right],
$$

converges in finite-dimensional distributions to a four-parameter family of continuum conditioned partition functions $\left\{\boldsymbol{Z}_{s, t ; \hat{\beta}}^{W, c}(x, y)\right\}_{(s, x),(t, y) \in \mathbb{R}^{+} \times \mathbb{R}}$.

Similar to the case $\alpha=2$ studied in AKQ14b, we expect that this convergence can be upgraded to a convergence in distribution in the space of continuous functions, equipped with the uniform topology. We can then use these partition functions to define a continuum long-range directed polymer model (which corresponds intuitively to an " $\alpha$-stable Lévy process in a white noise random medium"), by specifying its finite-dimensional distributions as done in AKQ14b for the Brownian case $\alpha=2$.

Remark 3.10. The free energy of the (discrete) directed polymer model is defined by

$$
F(\beta):=\lim _{N \rightarrow \infty} \frac{1}{N} \mathbb{E}\left[\log Z_{N, \beta}^{\omega}\right],
$$

where we expect that the limit exists (also $\mathbb{P}$-a.s. and in $L^{1}(\mathrm{dP})$ ), as in the usual setting. It is natural to define the free energy of the continuum model analogously, i.e.

$$
\boldsymbol{F}(\hat{\beta}):=\lim _{t \rightarrow \infty} \frac{1}{t} \mathbb{E}\left[\log \boldsymbol{Z}_{t, \hat{\beta}}^{W}\right],
$$

assuming of course that the limit exists. We stress that $\boldsymbol{F}(\cdot)$ is a universal quantity, which depends only on the parameters $\left(\alpha, \sigma^{2}\right)$ or $(\alpha, \gamma, C)$ in Assumption 3.7 (furthermore, the parameters $\sigma^{2}, C$ enter as simple scale factors). We also note that $\boldsymbol{F}(\hat{\beta})=\boldsymbol{F}(1) \hat{\beta}^{\frac{\alpha-1}{2 \alpha}}$, by an easy scaling argument. In analogy with Remark 3.4, we are led to the following 
Conjecture 3.11. For any directed polymer model satisfying Assumption [3.7, the free energy $F(\beta)$ has the following universal asymptotic behavior for weak disorder:

$$
\lim _{\beta \downarrow 0} \frac{F(\beta)}{\beta^{\frac{2 \alpha}{\alpha-1}}}=\lim _{\delta \downarrow 0} \frac{F\left(\delta^{\frac{\alpha-1}{2 \alpha}}\right)}{\delta}=\boldsymbol{F}(1) .
$$

When $\alpha=2$ we would then get $F(\beta) \sim \boldsymbol{F}(1) \beta^{4}$, which is supported by the (non-matching) upper and lower bounds on $F(\beta)$ obtained in [L10].

Remark 3.12. For $1<\alpha<2$, the function $g_{t}(\cdot)$ in (3.24) is the marginal density of the $\alpha$-stable Lévy process $\left(X_{t}\right)_{t \in \mathbb{R}^{+}}$whose infinitesimal generator is given by a multiple of

$$
\Delta^{\alpha / 2, \gamma} f(x):=\int_{-\infty}^{+\infty}\left(f(x+y)-f(x)-y f^{\prime}(x)\right)\left(\frac{1+\gamma}{|y|^{1+\alpha}} \mathbb{1}_{\{y>0\}}+\frac{1-\gamma}{|y|^{1+\alpha}} \mathbb{1}_{\{y<0\}}\right) \mathrm{d} y .
$$

In the symmetric case $\gamma=0$, this reduces to the much studied fractional Laplacian

$$
\Delta^{\alpha / 2} f(x):=\int_{-\infty}^{\infty}\left(f(x+y)-f(x)-y f^{\prime}(x)\right) \frac{1}{|y|^{1+\alpha}} \mathrm{d} y .
$$

Let us stick for simplicity to the symmetric case $\gamma=0$. It is natural to call $Z_{t, \hat{\beta}}^{W, c}(x)$ in (3.25) the continuum conditioned point-to-point partition function. Introducing the continuum constrained point-to-point partition function by

$$
Z_{t, \hat{\beta}}^{W}(x):=Z_{t, \hat{\beta}}^{W, c}(x) g_{t}(x),
$$

one can check that the process $u(t, x)=Z_{t, \hat{\beta}}^{W}(x)$ has a version that is continuous in $(t, x)$ and, up to a scaling factor, it is a mild solution to the one dimensional stochastic PDE

$$
\left\{\begin{array}{l}
\partial_{t} u=\Delta^{\alpha / 2} u+\sqrt{p} \hat{\beta} \dot{W} u \\
u(0, \cdot)=\delta_{0}(\cdot)
\end{array},\right.
$$

which we can call the stochastic fractional heat equation (SFHE), generalizing the usual SHE (which corresponds to $\alpha=2$ ). Uniqueness of mild solutions for the SFHE follows from standard techniques, see discussions in [CJKS14 and references therein.

Let us also consider the process $A_{\alpha, \hat{\beta}}(\cdot):=\log Z_{1, \hat{\beta}}^{W}(\cdot)$. When $\alpha=2$, this is the crossover process studied in ACQ11, SS10, which owes its name to the fact that its one-point marginals interpolate between the Gaussian distribution (in the limit $\hat{\beta} \rightarrow 0$ ) and the TracyWidom GUE distribution (in the limit $\hat{\beta} \rightarrow \infty$ ). When $\alpha<2$, it is easy to see that $A_{\alpha, \hat{\beta}}(\cdot)$ is again asymptotically Gaussian for $\hat{\beta} \rightarrow 0$ (the contribution from the first stochastic integral in its Wiener chaos expansion is dominant over the iterated integrals, which are multiplied by higher powers of $\hat{\beta}$ ). However, it is far from obvious whether $A_{\alpha, \hat{\beta}}(\cdot)$ converges to some asymptotic process $A_{\alpha, \infty}(\cdot)$ as $\hat{\beta} \rightarrow \infty$ and whether such a process describes some universality class for long-range random polymers, last passage percolation and growth models, generalizing the the so-called Airy process obtained for $\alpha=2$. Besides a very recent work on the limit shapes of long-range first-passage percolation model [CD13], long-range polymer type models do not appear to have been studied systematically before. 
3.3. RANDOM FIELD IsING MODEL. Given a bounded $\Omega \subseteq \mathbb{Z}^{2}$, we set

$$
\partial \Omega:=\left\{x \in \mathbb{Z}^{2} \backslash \Omega:\|x-y\|=1 \text { for some } y \in \Omega\right\} .
$$

For a fixed parameter $\beta \geq 0$, representing the "inverse temperature", the Ising model on $\Omega$ with + boundary condition (and zero external field) is the probability measure $\mathrm{P}_{\Omega}^{+}$on the set of spin configurations $\{ \pm 1\}^{\Omega}$, where each $\sigma:=\left(\sigma_{x}\right)_{x \in \Omega} \in\{ \pm 1\}^{\Omega}$ has probability

$$
\mathrm{P}_{\Omega}^{+}(\sigma):=\frac{1}{Z_{\Omega}^{+}} \exp \left\{\beta \sum_{x \sim y \in \Omega \cup \partial \Omega} \sigma_{x} \sigma_{y}\right\} \prod_{x \in \partial \Omega} \mathbb{1}_{\left\{\sigma_{x}=+1\right\}} .
$$

Here $x \sim y$ denotes an unordered nearest-neighbor pair in $\mathbb{Z}^{2}$, and $Z_{\Omega}^{+}$is the normalizing constant. The value of $\beta$ will soon be fixed, which is why we do not indicate it in $\mathrm{P}_{\Omega}^{+}$.

Remark 3.13. It is well-known that as $\Omega \uparrow \mathbb{Z}^{2}$, the sequence of probability measures $\mathrm{P}_{\Omega}^{+}$ has a unique infinite volume limit $\mathrm{P}_{\mathbb{Z}^{2}}^{+}$, which of course depends on $\beta$, such that

$$
\mathrm{E}_{\mathbb{Z}^{2}}^{+}\left(\sigma_{0}\right)>0 \quad \text { if and only if } \beta>\beta_{c}:=\frac{1}{2} \log (1+\sqrt{2}) .
$$

By an obvious symmetry, for $\beta>\beta_{c}$ there also exists an infinite volume Gibbs measure $\mathrm{P}_{\mathbb{Z}^{2}}^{-}$ satisfying $\mathrm{E}_{\mathbb{Z}^{2}}^{-}\left(\sigma_{0}\right)<0$. Given the coexistence of multiple infinite volume Gibbs measures, the Ising model is said to have a first order phase transition for $\beta>\beta_{c}$. The same result holds in higher dimensions, with different values of $\beta_{c}$.

A question that attracted significant interest was whether this picture will be altered by the addition of a small random external field. After long debates, this question was settled by Bricmont and Kupiainen [BK88], who showed that the first order phase transition persists for the random field Ising model in dimensions $d \geq 3$ at low temperatures (i.e., for large $\beta$ ), and by Aizenman and Wehr AW90 who showed the absence of first order phase transition in dimension 2 at any temperature. See [B06, Chap. 7] for an overview.

Henceforth we fix $\beta=\beta_{c}:=\frac{1}{2} \log (1+\sqrt{2})$, so that $\mathrm{P}_{\Omega}^{+}$denotes the two-dimensional critical Ising model. Let $\omega:=\left(\omega_{x}\right)_{x \in \mathbb{Z}^{2}}$ be a family of i.i.d. random variables satisfying (3.1), representing the disorder. Given $\lambda:=\left(\lambda_{x}\right)_{x \in \mathbb{Z}^{2}} \geq 0$ and $h:=\left(h_{x}\right)_{x \in \mathbb{Z}^{2}} \in \mathbb{R}$, representing the disorder strength and bias respectively, the random field Ising model (RFIM) is the probability measure $\mathrm{P}_{\Omega, \lambda, h}^{+, \omega}$ on $\{ \pm 1\}^{\Omega}$ with

$$
\mathrm{P}_{\Omega, \lambda, h}^{+, \omega}(\sigma)=\frac{1}{Z_{\Omega, \lambda, h}^{+, \omega}} \exp \left\{\sum_{x \in \Omega}\left(\lambda_{x} \omega_{x}+h_{x}\right) \sigma_{x}\right\} \mathrm{P}_{\Omega}^{+}(\sigma),
$$

where the normalizing constant, called partition function, is given by

$$
Z_{\Omega, \lambda, h}^{+, \omega}=\mathrm{E}_{\Omega}^{+}\left[\exp \left\{\sum_{x \in \Omega}\left(\lambda_{x} \omega_{x}+h_{x}\right) \sigma_{x}\right\}\right] .
$$

Note that we allow the disorder strength $\lambda$ and bias $h$ to vary from site to site. Also observe that choosing $\mathrm{P}_{\Omega}^{+}$as a "reference law" means that $Z_{\Omega, \lambda, h}^{+, \omega}=1$ for $\lambda, h \equiv 0$ (with $\beta=\beta_{c}$ ).

Fix now a bounded open set $\Omega \subseteq \mathbb{R}^{2}$ with piecewise smooth boundary, and define the rescaled lattice $\Omega_{\delta}:=\Omega \cap(\delta \mathbb{Z})^{2}$, for $\delta>0$. We are going to obtain a non-trivial limit in distribution for the partition function $Z_{\Omega_{\delta}, \lambda, h}^{+, \omega}$, in the continuum and weak disorder regime 
$\delta, \lambda, h \rightarrow 0$. We build on recent results of Chelkak, Hongler and Izyurov [CHI12, Theorem 1.3] on the continuum limit of the spin correlations under $\mathrm{P}_{\Omega_{\delta}}^{+}$(the two-dimensional critical Ising model with + boundary condition): for all $n \in \mathbb{N}$ and distinct $x_{1}, \ldots, x_{n} \in \Omega$

$$
\lim _{\delta \downarrow 0} \delta^{-\frac{n}{8}} \mathrm{E}_{\Omega_{\delta}}^{+}\left[\sigma_{x_{1}} \cdots \sigma_{x_{n}}\right]=\mathcal{C}^{n} \phi_{\Omega}^{+}\left(x_{1}, \ldots, x_{n}\right),
$$

where $\phi_{\Omega}^{+}: \cup_{n \in \mathbb{N}} \Omega^{n} \rightarrow \mathbb{R}$ is a symmetric function and $\mathcal{C}:=2^{\frac{5}{48}} e^{-\frac{3}{2} \zeta^{\prime}(-1)}$, with $\zeta^{\prime}$ denoting the derivative of Riemann's zeta function (For simplicity, in (3.33) we have set $\sigma_{x}:=\sigma_{x_{\delta}}$, where $x_{\delta}$ denotes the point in $\Omega_{\delta}$ closest to $x \in \Omega$.)

We can complement (3.33) with a uniform bound (see Lemma 8.1 below): there exists $C \in(0, \infty)$ such that for all $n \in \mathbb{N}, x_{1}, \ldots, x_{n} \in \Omega$ and $\delta \in(0,1)$

$$
0 \leq \delta^{-\frac{n}{8}} \mathrm{E}_{\Omega_{\delta}}^{+}\left[\sigma_{x_{1}} \cdots \sigma_{x_{n}}\right] \leq \prod_{i=1}^{n} \frac{C}{d\left(x_{i}, \partial \Omega \cup\left\{x_{1}, \ldots, x_{n}\right\} \backslash\left\{x_{i}\right\}\right)^{\frac{1}{8}}},
$$

where $d(x, A):=\inf _{y \in A}\|x-y\|$ and the RHS is shown to be in $L^{2}$ in Lemma 8.3 below. Therefore the convergence (3.33) also holds in $L^{2}(\Omega)$, and $\left\|\phi_{\Omega}^{+}\right\|_{L^{2}\left(\Omega^{n}\right)}<\infty$ for every $n \in \mathbb{N}$.

We can now state our main result for the RFIM, to be proved in Section 8 ,

Theorem 3.14 (Scaling limits of RFIM). Let $\Omega \subseteq \mathbb{R}^{2}$ be bounded and simply connected open set with piecewise smooth boundary, and define $\Omega_{\delta}:=\Omega \cap(\delta \mathbb{Z})^{2}$, for $\delta>0$.

Let $\omega:=\left(\omega_{x}\right)_{x \in \mathbb{Z}^{2}}$ be i.i.d. random variables satisfying (3.1) and define $\omega_{\delta}=\left(\omega_{\delta, x}\right)_{x \in \Omega_{\delta}}$ by $\omega_{\delta, x}:=\omega_{x / \delta}$. Given two continuous functions $\hat{\lambda}: \bar{\Omega} \rightarrow(0, \infty)$ and $\hat{h}: \bar{\Omega} \rightarrow \mathbb{R}$, define

$$
\lambda_{\delta, x}:=\hat{\lambda}(x) \delta^{\frac{7}{8}}, \quad h_{\delta, x}:=\hat{h}(x) \delta^{\frac{15}{8}} .
$$

and set $\lambda_{\delta}=\left(\lambda_{\delta, x}\right)_{x \in \Omega_{\delta}}, h_{\delta}=\left(h_{\delta, x}\right)_{x \in \Omega_{\delta}}$. Then the rescaled partition function

$$
e^{-\frac{1}{2}\|\hat{\lambda}\|_{L^{2}(\Omega)}^{2} \delta^{-1 / 4}} Z_{\Omega_{\delta}, \lambda_{\delta}, h_{\delta}}^{+, \omega_{\delta}}
$$

converges in distribution as $\delta \downarrow 0$ to a random variable $\boldsymbol{Z}_{\Omega, \hat{\lambda}, \hat{h}}^{+, W}$ with Wiener chaos expansion

$$
Z_{\Omega, \hat{\lambda}, \hat{h}}^{+, W}=1+\sum_{n=1}^{\infty} \frac{\mathcal{C}^{n}}{n !} \int \cdots \int_{\Omega^{n}} \phi_{\Omega}^{+}\left(x_{1}, \ldots, x_{n}\right) \prod_{i=1}^{n}\left[\hat{\lambda}\left(x_{i}\right) W\left(\mathrm{~d} x_{i}\right)+\hat{h}\left(x_{i}\right) \mathrm{d} x_{i}\right],
$$

where $W(\cdot)$ denotes white noise on $\mathbb{R}^{2}$ and $\phi_{\Omega}^{+}(\cdot), \mathcal{C}$ are as in (3.33).

Remark 3.15. We impose the continuity on $\hat{\lambda}$ and $\hat{h}$ and the strict positivity on $\hat{\lambda}$ for technical simplicity: these conditions can be relaxed with a more careful analysis.

We call the random variable $\boldsymbol{Z}_{\Omega, \hat{\lambda}, \hat{h}}^{+, W}$ in (3.37) the continuum RFIM partition function. The fact that the continuum correlation function $\phi_{\Omega}^{+}$is conformally covariant, proved in [CHI12, Theorem 1.3], allows to deduce the conformal covariance of $\boldsymbol{Z}_{\Omega, \hat{\lambda}, \hat{h}}^{+, W}$.

${ }^{\dagger}$ In CHI12, the mesh size of $\Omega_{\delta}$ is $\sqrt{2} \delta$ instead of $\delta$, which accounts for the difference between our formula for $\mathcal{C}$ and that in [CHI12, (1.3)]. Exact formulas for $\phi_{\Omega}^{+}\left(x_{1}, \ldots, x_{n}\right)$ are available for $n=1,2$ when $\Omega$ is the upper half plane $\mathbb{H}$, cf. [CHI12, (1.4)]. The general case $\phi_{\Omega}^{+}$can be obtained from $\phi_{\mathbb{H}}^{+}$via conformal map. 
Corollary 3.16 (Conformal covariance). Let $\Omega, \widetilde{\Omega} \subseteq \mathbb{R}^{2}$ be two bounded and simply connected open sets with piecewise smooth boundaries, and let $\varphi: \widetilde{\Omega} \rightarrow \Omega$ be conformal. Let $\hat{\lambda}: \bar{\Omega} \rightarrow(0, \infty)$ and $\hat{h}: \bar{\Omega} \rightarrow \mathbb{R}$ be continuous functions. Then

$$
\boldsymbol{Z}_{\Omega, \hat{\lambda}, \hat{h}}^{+, W} \stackrel{d}{=} \boldsymbol{Z}_{\widetilde{\Omega}, \widetilde{\lambda}, \widetilde{h}}^{+, W}
$$

where we set $\widetilde{\lambda}(z):=\left|\varphi^{\prime}(z)\right|^{\frac{7}{8}} \hat{\lambda}(\varphi(z))$ and $\widetilde{h}(z):=\left|\varphi^{\prime}(z)\right|^{\frac{15}{8}} \hat{h}(\varphi(z))$ for all $z \in \widetilde{\Omega}$.

Remark 3.17. Recently, Camia, Garban and Newman [CGN12, CGN13] showed that for a deterministic external field, more precisely $\lambda_{\delta, x} \equiv 0$ and $h_{\delta, x} \equiv \hat{h} \delta^{\frac{15}{8}}$ for fixed $\hat{h} \in \mathbb{R}$, the Ising measure $\mathrm{P}_{\Omega_{\delta}, \lambda_{\delta}, h_{\delta}}^{+}$converges as $\delta \downarrow 0$ (in a suitable sense) to a limiting distributionvalued process $\Phi_{\Omega}^{\infty}, \hat{h}$. Theorem 3.14 can be regarded as a first step towards the extension of this convergence to the case of a random external field, where $\boldsymbol{Z}_{\Omega, \hat{\lambda}, \hat{h}}^{+, W}$ plays the role of the partition function of a continuum Ising model with random external field $(\hat{\lambda}(x) W(\mathrm{~d} x)+\hat{h}(x) \mathrm{d} x)$ and + boundary condition. The next step towards the construction of such a continuum model would be to identify the joint distribution of the partition functions $\left(\boldsymbol{Z}_{\Gamma, \hat{\lambda}, \hat{h}, \hat{h}}^{\mathrm{b}, W}\right)_{\Gamma, \mathrm{b}}$ (note that they are random variables which are functions of the disorder $W$ ) for a large enough family of sub-domains $\Gamma \subseteq \Omega$ and "boundary conditions" b.

Remark 3.18. Consider the case when the disorder strength and bias are constant, i.e. $\lambda_{x} \equiv \lambda \geq 0$ and $h_{x} \equiv h \in \mathbb{R}$. The free energy $F(\lambda, h)$ of the critical random field Ising model can be defined as follows: setting $\Omega:=\left(-\frac{1}{2}, \frac{1}{2}\right)^{2}$ and $\Lambda_{N}:=(N \Omega) \cap \mathbb{Z}^{2}=\left\{\left\lceil-\frac{N}{2}\right\rceil, \ldots,\left\lfloor\frac{N}{2}\right\rfloor\right\}^{2}$,

$$
F(\lambda, h):=\lim _{N \rightarrow \infty} \frac{1}{N^{2}|\Omega|} \mathbb{E}\left[\log Z_{\Lambda_{N}, \lambda, h}^{+, \omega}\right]=\lim _{\delta \downarrow 0} \frac{\delta^{2}}{|\Omega|} \mathbb{E}\left[\log Z_{\Omega_{\delta}, \lambda, h}^{+, \omega}\right],
$$

where the limit exists by standard super-additivity arguments and is independent of the choice of $\Omega$. Note that $F(0,0)=0$, that is $F(\lambda, h)$ represents the excess free energy with respect to the critical Ising model, cf. (3.32).

It is natural to define a continuum free energy $\boldsymbol{F}(\hat{\lambda}, \hat{h})$ for $\hat{\lambda} \geq 0, \hat{h} \in \mathbb{R}$ by

$$
\boldsymbol{F}(\hat{\lambda}, \hat{h}):=\lim _{\Omega \uparrow \mathbb{R}^{2}} \frac{1}{\operatorname{Leb}(\Omega)} \mathbb{E}\left[\log \boldsymbol{Z}_{\Omega, \hat{\lambda}, \hat{h}}^{+, W}\right],
$$

provided the limit exists (at least along sufficiently nice domains $\Omega \uparrow \mathbb{R}^{2}$ ), where $\mathbb{E}$ denotes expectation with respect to the white noise $W(\cdot)$. In analogy with Remark 3.4, Theorem 3.14 suggests the following conjecture on the universal behavior of the free energy $F(\lambda, h)$ in the weak disorder regime $\lambda, h \rightarrow 0$.

Conjecture 3.19. The following asymptotic relation holds:

$$
\lim _{\delta \downarrow 0} \frac{F\left(\hat{\lambda} \delta^{\frac{7}{8}}, \hat{h} \delta^{\frac{15}{8}}\right)}{\delta^{2}}=\boldsymbol{F}(\hat{\lambda}, \hat{h}), \quad \forall \hat{\lambda} \geq 0, \hat{h} \in \mathbb{R} .
$$

One can go one step further: differentiating both sides of (3.39) with respect to $\hat{h}$ and setting $\delta=h^{\frac{8}{15}}$, for $\hat{h}=1$ one would obtain

$$
\lim _{h \rightarrow 0} \frac{\left\langle\sigma_{0}\right\rangle_{\hat{\lambda} h \frac{7}{15}, h}}{h^{\frac{1}{15}}}=\frac{\partial \boldsymbol{F}}{\partial \hat{h}}(\hat{\lambda}, 1),
$$


where $\left\langle\sigma_{0}\right\rangle_{\lambda, h}:=\frac{\partial F}{\partial h}(\lambda, h)=\lim _{N \rightarrow \infty} \mathbb{E}\left[\mathrm{E}_{\Lambda_{N}, \lambda, h}^{+, \omega}\left[\sigma_{0}\right]\right]$ represents the average magnetization in the infinite-volume random field critical Ising model, cf. (3.38) and (3.31). Relation (3.40) is supported by (non-matching) upper and lower bounds of the order $h^{\frac{1}{15}}$ for $\left\langle\sigma_{0}\right\rangle_{\lambda, h}$ in the non-disordered case $\lambda=0$, recently proved by Camia, Garban and Newman [CGN12b.

In light of Theorem 3.14, and the fact that for the two-dimensional Ising model below the critical temperature a disordered external field smoothens the phase transition [AW90, it is natural to conjecture that a similar smoothing effect occurs at the critical temperature:

Conjecture 3.20. For any fixed $\lambda>0$, the average magnetization in the infinite-volume random field critical Ising model on $\mathbb{Z}^{2}$ satisfies

$$
\left\langle\sigma_{0}\right\rangle_{\lambda, h} \sim C h^{\gamma} \quad \text { as } h \downarrow 0 \quad \text { for some } \gamma>\frac{1}{15} .
$$

\section{Proof of the Lindeberg Principle}

In this section, we prove Theorems 2.6 and 2.8 on the Lindeberg principle for polynomial chaos expansions. We first deduce Theorem 2.8 from Theorem 2.6, starting with the following lemma which controls how a multi-linear polynomial $\Psi(x)=\Psi\left(\left(x_{i}\right)_{i \in \mathbb{T}}\right)$ is affected by a shift $x \mapsto x+\mu$, where $\mu:=\left(\mu_{i}\right)_{i \in \mathbb{T}}$.

Lemma 4.1 (Effect of adding a mean). Let $\Psi(x)=\Psi\left(\left(x_{i}\right)_{i \in \mathbb{T}}\right)$ be a multi-linear polynomial as in (2.5), with kernel $\psi$, and let $\mu:=\left(\mu_{i}\right)_{i \in \mathbb{T}}$ be a family of real numbers. Then $\widetilde{\Psi}(x):=\Psi(x+\mu)$ is a multi-linear polynomial, i.e. $\widetilde{\Psi}(x)=\sum_{J \in \mathcal{P}^{\mathrm{fin}}(\mathbb{T})} \widetilde{\psi}(J) x^{J}$, with kernel

$$
\widetilde{\psi}(J)=\sum_{I \in \mathcal{P}^{\mathrm{fin}}(\mathbb{T}): I \supseteq J} \psi(I) \mu^{I \backslash J} .
$$

For $\varepsilon>0$, let $\Psi^{(\varepsilon)}(x)$ be defined as in (2.26), and recall the definitions of $\mathrm{C}_{\Psi}$ from (2.19) and $\operatorname{Inf}_{j}[\Psi]$ from (2.20). Then, setting $\mathrm{c}_{\mu}:=\sum_{i \in \mathbb{T}} \mu_{i}^{2}$, for any $\varepsilon>0$ we have

$$
\mathrm{C}_{\widetilde{\Psi}} \leq e^{\mathrm{c}_{\mu} / \varepsilon} \mathrm{C}_{\Psi^{(\varepsilon)}}, \quad \operatorname{Inf}_{j}[\widetilde{\Psi}] \leq e^{c_{\mu} / \varepsilon} \operatorname{Inf}_{j}\left[\Psi^{(\varepsilon)}\right]
$$

Proof. Note that (4.1) follows from the expansion

$$
\widetilde{\Psi}(x)=\Psi(x+\mu)=\sum_{I} \psi(I)(x+\mu)^{I}=\sum_{I} \psi(I) \sum_{J \subseteq I} \mu^{I \backslash J} x^{J}=\sum_{J}\left(\sum_{I \supseteq J} \psi(I) \mu^{I \backslash J}\right) x^{J} .
$$

For any $\varepsilon>0$, we can apply Cauchy-Schwarz to write

$$
\widetilde{\psi}(J)^{2} \leq\left(\sum_{I \supseteq J}\left(\varepsilon^{-1} \mu^{2}\right)^{I \backslash J}\right)\left(\sum_{I \supseteq J} \varepsilon^{|I \backslash J|} \psi(I)^{2}\right)=\left(1+\varepsilon^{-1} \mu^{2}\right)^{\mathbb{T} \backslash J}\left(\sum_{I \supseteq J} \varepsilon^{|I \backslash J|} \psi(I)^{2}\right),
$$

and note that

$$
\left(1+\varepsilon^{-1} \mu^{2}\right)^{\mathbb{T} \backslash J} \leq\left(1+\varepsilon^{-1} \mu^{2}\right)^{\mathbb{T}} \leq e^{c_{\mu} / \varepsilon}
$$

Therefore

$$
\mathrm{C}_{\widetilde{\Psi}}:=\sum_{J} \widetilde{\psi}(J)^{2} \leq e^{\mathrm{c}_{\mu} / \varepsilon} \sum_{I}\left(\sum_{J \subseteq I} \varepsilon^{|I \backslash J|}\right) \psi(I)^{2}=e^{\mathrm{c}_{\mu} / \varepsilon} \sum_{I}(1+\varepsilon)^{|I|} \psi(I)^{2}=: e^{\mathrm{c}_{\mu} / \varepsilon} \mathrm{C}_{\Psi^{(\varepsilon)}},
$$


proving the first relation in (4.2). The second relation is obtained similarly:

$$
\begin{aligned}
\operatorname{Inf}_{j}[\widetilde{\Psi}]:=\sum_{J \ni j} \widetilde{\psi}(J)^{2} & \leq e^{c_{\mu} / \varepsilon} \sum_{I \ni j}\left(\sum_{J: j \in J \subseteq I} \varepsilon^{|I \backslash J|}\right) \psi(I)^{2} \\
& \leq e^{c_{\mu} / \varepsilon} \sum_{I \ni j}(1+\varepsilon)^{|I|} \psi(I)^{2}=: e^{c_{\mu} / \varepsilon} \operatorname{Inf}_{j}\left[\Psi^{(\varepsilon)}\right] .
\end{aligned}
$$

This concludes the proof of the lemma.

Proof of Theorem 2.8. Since $\Psi(\widetilde{\zeta})=\Psi(\zeta+\mu)=$ : $\widetilde{\Psi}(\zeta)$, by Lemma 4.1 the conditions $\mathrm{c}_{\mu}<\infty$ and $\mathrm{C}_{\Psi^{(\varepsilon)}}<\infty$ ensure that $\mathrm{C}_{\widetilde{\Psi}}<\infty$. The polynomial chaos expansion $\Psi(\widetilde{\zeta})$ is then a well-defined $L^{2}$ random variable, by Remark 2.2, and the same holds for $\Psi(\widetilde{\xi})$.

To prove (2.27), we are first going to truncate $\Psi$ to degree $\ell$, i.e. we consider $\Psi^{\leq \ell}$ and $\Psi^{>\ell}$, defined in (2.21). Note that

$$
|\mathbb{E}[f(\Psi(\widetilde{\zeta}))-f(\Psi \leq \ell(\widetilde{\zeta}))]| \leq\left\|f^{\prime}\right\|_{\infty} \mathbb{E}[|\Psi(\widetilde{\zeta})-\Psi \leq \ell(\widetilde{\zeta})|] \leq\left\|f^{\prime}\right\|_{\infty} \mathbb{E}\left[\left|\Psi^{>\ell}(\widetilde{\zeta})\right|^{2}\right]^{\frac{1}{2}}
$$

and the same bound holds when $\widetilde{\zeta}$ is replaced by $\widetilde{\xi}$, therefore

$$
|\mathbb{E}[f(\Psi(\widetilde{\zeta}))]-\mathbb{E}[f(\Psi(\widetilde{\xi}))]| \leq 2\left\|f^{\prime}\right\|_{\infty} \mathbb{E}\left[\left|\Psi^{>\ell}(\widetilde{\zeta})\right|^{2}\right]^{\frac{1}{2}}+\left|\mathbb{E}\left[f\left(\Psi^{\leq \ell}(\widetilde{\zeta})\right)\right]-\mathbb{E}\left[f\left(\Psi^{\leq \ell}(\widetilde{\xi})\right)\right]\right|
$$

where we use the fact that, since $\zeta$ and $\xi$ are independent with zero mean,

$$
\mathbb{E}\left[\left|\Psi^{>\ell}(\zeta)\right|^{2}\right]=\mathbb{E}\left[\left|\Psi^{>\ell}(\xi)\right|^{2}\right]=\sum_{|I|>\ell} \psi(I)^{2}=\mathrm{C}_{\Psi>\ell}
$$

To bound the first term in (4.6), we write $\widetilde{\Psi}^{>\ell}(x):=\Psi^{>\ell}(x+\mu)$, which has kernel $\widetilde{\psi}^{>\ell}$ (note that first we truncate the kernel and then we shift $x \mapsto x+\mu$ ). Since $\zeta$ are independent with zero mean and variance one, by Lemma 4.1 we have

$$
\mathbb{E}\left[\left|\Psi^{>\ell}(\widetilde{\zeta})\right|^{2}\right]=\mathbb{E}\left[\left|\widetilde{\Psi}^{>\ell}(\zeta)\right|^{2}\right]=\sum_{J} \widetilde{\psi}^{>\ell}(J)^{2}=: \mathrm{C}_{\widetilde{\Psi}>\ell} \leq e^{c_{\mu} / \varepsilon} C_{\Psi^{(\varepsilon),>\ell}}
$$

Substituting this bound into (4.6) then leads to the first term in (2.27).

To bound the second term in (4.6), we write $\widetilde{\Psi}^{\leq \ell}(x):=\Psi^{\leq \ell}(x+\mu)$, and then apply Theorem 2.6 to obtain

$$
\begin{aligned}
\left|\mathbb{E}\left[f\left(\Psi^{\leq \ell}(\widetilde{\zeta})\right)\right]-\mathbb{E}\left[f\left(\Psi^{\leq \ell}(\widetilde{\xi})\right)\right]\right| & =\left|\mathbb{E}\left[f\left(\widetilde{\Psi}^{\leq \ell}(\zeta)\right)\right]-\mathbb{E}\left[f\left(\widetilde{\Psi}^{\leq \ell}(\xi)\right)\right]\right| \\
& \leq C_{f} C_{\widetilde{\Psi} \leq \ell}\left(16 \ell^{2} m_{2}^{>M}+70^{\ell+1}\left(m_{3}^{\leq M}\right)^{\ell} \max _{i \in \mathbb{T}} \sqrt{\operatorname{Inf}_{i}[\widetilde{\Psi} \leq \ell]}\right) .
\end{aligned}
$$

Applying the bounds in Lemma 4.1 to $\Psi \leq \ell$ then gives the remaining terms in (2.27), where we have combined and upper bounded factors of $e^{c_{\mu} / \varepsilon}$.

Proof of Theorem 2.6. We note that it is sufficient to prove the theorem in the case $|\mathbb{T}|<\infty$, because the general case follows by considering finite $\Lambda_{N} \uparrow \mathbb{T}$. For notational simplicity, we assume that $\mathbb{T}=[N]:=\{1, \ldots, N\}$.

Step 1. We first truncate $\Psi$ to a degree $\ell$ polynomial $\Psi^{\leq \ell}$. By the same calculations as in (4.5) and (4.6), we have

$$
|\mathbb{E}[f(\Psi(\zeta))]-\mathbb{E}[f(\Psi(\xi))]| \leq 2\left\|f^{\prime}\right\|_{\infty} \mathbb{E}\left[\left|\Psi^{>\ell}(\zeta)\right|^{2}\right]^{\frac{1}{2}}+\left|\mathbb{E}\left[f\left(\Psi^{\leq \ell}(\zeta)\right)\right]-\mathbb{E}\left[f\left(\Psi^{\leq \ell}(\xi)\right)\right]\right|
$$

This leads to the first term in the right hand side of (2.24). 
Step 2. For a fixed $f \in C_{b}^{3}(\mathbb{R})$, we denote

$$
f(\Psi \leq \ell(x))=: g\left(x_{1}, x_{2}, \ldots, x_{N}\right) .
$$

For a vector $x \in \mathbb{R}^{N}$ and $y \in \mathbb{R}$, we also set

$$
h_{j}^{x}(y)=g\left(x_{1}, \ldots, x_{j-1}, y, x_{j+1}, \ldots, x_{N}\right) .
$$

Using this notation, we can write

$$
\begin{aligned}
f\left(\Psi^{\leq \ell}(\zeta)\right)-f\left(\Psi^{\leq \ell}(\xi)\right) & =\sum_{j=1}^{N} g\left(\zeta_{1}, \ldots, \zeta_{j}, \xi_{j+1}, \ldots, \xi_{N}\right)-g\left(\zeta_{1}, \ldots, \zeta_{j-1}, \xi_{j}, \ldots, \xi_{N}\right) \\
& =\sum_{j=1}^{N}\left(h_{j}^{X^{j}}\left(\zeta_{j}\right)-h_{j}^{X^{j}}\left(\xi_{j}\right)\right),
\end{aligned}
$$

where we have used the notation

$$
X^{j}:=\left(X_{1}^{j}, \ldots, X_{N}^{j}\right):=\left(\zeta_{1}, \ldots, \zeta_{j}, \xi_{j+1}, \ldots, \xi_{N}\right) .
$$

Next, we will be Taylor expanding each term in (4.8). For this we note that for $y \in \mathbb{R}$

$$
h_{j}^{x}(y)=h_{j}^{x}(0)+y \frac{\mathrm{d} h_{j}^{x}}{\mathrm{~d} y}(0)+\frac{y^{2}}{2} \frac{\mathrm{d}^{2} h_{j}^{x}}{\mathrm{~d} y^{2}}(0)+R_{j}^{x}(y),
$$

where the remainder term has the form

$$
R_{j}^{x}(y)=\frac{1}{2} \int_{0}^{y} \frac{\mathrm{d}^{3} h_{j}^{x}}{\mathrm{~d} y^{3}}(t)(y-t)^{2} \mathrm{~d} t=\frac{1}{2} \frac{\mathrm{d}^{2} h_{j}^{x}}{\mathrm{~d} y^{2}}(0) y^{2}+\int_{0}^{y} \frac{\mathrm{d}^{2} h_{j}^{x}}{\mathrm{~d} y^{2}}(t)(y-t) \mathrm{d} t,
$$

from which it follows that

$$
\left|R_{j}^{x}(y)\right| \leq \min \left\{\frac{|y|^{3}}{6}\left\|\frac{\mathrm{d}^{3} h_{j}^{x}}{\mathrm{~d} y^{3}}\right\|_{\infty}, y^{2}\left\|\frac{\mathrm{d}^{2} h_{j}^{x}}{\mathrm{~d} y^{2}}\right\|_{\infty}\right\} .
$$

Inserting the Taylor expansion into (4.8) we obtain

$$
\begin{aligned}
f\left(\Psi^{\leq \ell}(\zeta)\right)-f\left(\Psi^{\leq \ell}(\xi)\right)=\sum_{j=1}^{N}\{ & h_{j}^{X^{j}}(0)+\zeta_{j} \frac{\mathrm{d} h_{j}^{X^{j}}}{\mathrm{~d} y}(0)+\frac{\zeta_{j}^{2}}{2} \frac{\mathrm{d}^{2} h_{j}^{X^{j}}}{\mathrm{~d} y^{2}}(0)+R_{j}^{X^{j}}\left(\zeta_{j}\right) \\
& \left.\quad-h_{j}^{X^{j}}(0)-\xi_{j} \frac{\mathrm{d} h_{j}^{X^{j}}}{\mathrm{~d} y}(0)-\frac{\xi_{j}^{2}}{2} \frac{\mathrm{d}^{2} h_{j}^{X^{j}}}{\mathrm{~d} y^{2}}(0)-R_{j}^{X^{j}}\left(\xi_{j}\right)\right\} .
\end{aligned}
$$

Since $\zeta_{i}$ and $\xi_{i}$ both have zero mean and unit variance, taking expectation, we get

$$
\begin{aligned}
\left|\mathbb{E}\left[f\left(\Psi^{\leq \ell}(\zeta)\right)\right]-\mathbb{E}\left[f\left(\Psi^{\leq \ell}(\xi)\right)\right]\right| & =\left|\sum_{j=1}^{N} \mathbb{E}\left[R_{j}^{X^{j}}\left(\zeta_{j}\right)-R_{j}^{X^{j}}\left(\xi_{j}\right)\right]\right| \\
& \leq \sum_{j=1}^{N} \mathbb{E}\left[\left|R_{j}^{X^{j}}\left(\zeta_{j}\right)\right|\right]+\sum_{j=1}^{N} \mathbb{E}\left[\left|R_{j}^{X^{j}}\left(\xi_{j}\right)\right|\right] .
\end{aligned}
$$

Since the estimates for both sums are identical, we will focus on the first one. 
Step 3. The derivatives of $h_{j}^{x}(\cdot)$ are easily computed:

$$
\begin{aligned}
\frac{\mathrm{d}^{m} h_{j}^{x}}{\mathrm{~d} y^{m}}(y) & =f^{(m)}\left(\Psi^{\leq \ell}\left(x_{1}, \ldots, x_{j-1}, y, x_{j+1}, \ldots, x_{N}\right)\right)\left(\frac{\partial \Psi \leq \ell}{\left(x_{1}, \ldots, x_{j-1}, y, x_{j+1}, \ldots, x_{N}\right)}\right)^{m} \\
& =f^{(m)}\left(\Psi^{\leq \ell}\left(x_{1}, \ldots, x_{j-1}, y, x_{j+1}, \ldots, x_{N}\right)\right)\left(\sum_{I \ni j,|I| \leq \ell} \psi(I) x^{I \backslash\{j\}}\right)^{m} .
\end{aligned}
$$

Then, setting $C_{f}=\max \left\{\left\|f^{\prime}\right\|_{\infty},\left\|f^{(2)}\right\|_{\infty},\left\|f^{(3)}\right\|_{\infty}\right\}$, we can apply (4.10) to bound

$$
\left.\sum_{j=1}^{N} \mathbb{E}\left[\mid R_{j}^{X^{j}}\left(\zeta_{j}\right)\right]\right] \leq C_{f} \sum_{j=1}^{N} \mathbb{E}\left[\varphi\left(L_{j}\left(X^{j}\right)\right)\right],
$$

where $X^{j}$ was introduced in (4.9), and we define

$$
\varphi(x):=\min \left\{\frac{|x|^{3}}{6},|x|^{2}\right\}, \quad L_{j}(x):=\sum_{I \ni j,|I| \leq \ell} \psi(I) x^{I} .
$$

Notice that $L_{j}(x)$ includes the variable $x_{j}$ in the product as a result of absorbing the powers of $y$ in (4.10). Also note that $\varphi(x)=\varphi(|x|)$ and

$$
\varphi(a+b) \leq \varphi(2 \max \{|a|,|b|\}) \leq \varphi(2|a|)+\varphi(2|b|) \leq 4|a|^{2}+\frac{4}{3}|b|^{3} .
$$

Writing $L_{j}\left(X^{j}\right)=\left(L_{j}\left(X^{j}\right)-L_{j}\left(X^{j-}\right)\right)+L_{j}\left(X^{j-}\right)$, where $X^{j-}:=\left(X_{1}^{j-}, \ldots, X_{N}^{j-}\right)$ is a suitably truncated version of $X^{j}$, we then obtain

$$
\mathbb{E}\left[\varphi\left(L_{j}\left(X^{j}\right)\right)\right] \leq 4 \mathbb{E}\left[\left(L_{j}\left(X^{j}\right)-L_{j}\left(X^{j-}\right)\right)^{2}\right]+\frac{4}{3} \mathbb{E}\left[\left|L_{j}\left(X^{j-}\right)\right|^{3}\right] .
$$

The two terms in the right hand side will give rise respectively to the two terms in the right hand side of (2.24).

Step 4. We now describe the truncation procedure. This new ingredient, with respect to MOO10, is tailored to control random variables with finite second moments and uniformly integrable squares. Fix $M \in(0, \infty)$. We want to decompose any real-valued random variable $Y$ with zero mean and finite variance in the following way:

$$
Y=Y^{-}+Y^{+},
$$

where $Y^{-}, Y^{+}$are functions of $Y$ and possibly of some extra randomness, such that

$$
\begin{aligned}
& \mathbb{E}\left[Y^{-}\right]=\mathbb{E}\left[Y^{+}\right]=0, \quad Y^{-} Y^{+}=0, \\
& \left|Y^{-}\right| \leq|Y| \mathbb{1}_{\{|Y| \leq M\}}, \quad \mathbb{E}\left[\left(Y^{+}\right)^{2}\right] \leq 2 \mathbb{E}\left[Y^{2} \mathbb{1}_{\{|Y|>M\}}\right] .
\end{aligned}
$$

If $\mathbb{E}\left[Y \mathbb{1}_{\{-M \leq Y \leq M\}}\right]=0$ we are done: just choose $Y^{-}:=Y \mathbb{1}_{\{-M \leq Y \leq M\}}$ and $Y^{+}:=Y-Y^{-}$. If, on the other hand, $\mathbb{E}\left[Y \mathbb{1}_{\{-M \leq Y \leq M\}}\right]>0$ (the strictly negative case is analogous), we set

$$
\bar{T}:=\sup \left\{T \in[0, M]: \mathbb{E}\left[Y \mathbb{1}_{\{-M \leq Y \leq T\}}\right] \leq 0\right\} \in[0, M] .
$$

Note that $\mathbb{E}\left[Y \mathbb{1}_{\{-M \leq Y \leq \bar{T}\}}\right] \geq 0$, because $T \mapsto \mathbb{E}\left[Y \mathbb{1}_{\{-M \leq Y \leq T\}}\right]$ is right-continuous. If $\mathbb{E}\left[Y \mathbb{1}_{\{-M \leq Y \leq \bar{T}\}}\right]=0$, defining $Y^{-}:=Y \mathbb{1}_{\{-M \leq Y \leq \bar{T}\}}$ and $Y^{+}:=Y-Y^{-}$, all the properties in (4.16) are clearly satisfied, except the last one that will be checked below. Finally, we consider the case $\mathbb{E}\left[Y \mathbb{1}_{\{-M \leq Y \leq \bar{T}\}}\right]>0$ (then necessarily $\bar{T}>0$ ). Since $\mathbb{E}\left[Y \mathbb{1}_{\{-M \leq Y<\bar{T}\}}\right] \leq 0$ 
by definition of $\bar{T}$, we must have $\mathbb{P}(Y=\bar{T})>0$. Then take a random variable $U$ uniformly distributed in $(0,1)$ and independent of $Y$, and define

$$
Y^{-}:=Y\left(\mathbb{1}_{\{-M \leq Y<\bar{T}\}}+\mathbb{1}_{\{Y=\bar{T}, U \leq \varrho\}}\right), \quad \text { where } \quad \varrho:=\frac{-\mathbb{E}\left[Y \mathbb{1}_{\{-M \leq Y<\bar{T}\}}\right]}{\bar{T} \mathbb{P}(Y=\bar{T})} \in(0,1) .
$$

Setting $Y^{+}:=Y-Y^{-}$, all the properties (4.16) but the last one are clearly satisfied.

For the last property, we write

$\mathbb{E}\left[\left(Y^{+}\right)^{2}\right]=\mathbb{E}\left[\left(Y^{+}\right)^{2} \mathbb{1}_{\{|Y|>M\}}\right]+\mathbb{E}\left[\left(Y^{+}\right)^{2} \mathbb{1}_{\{|Y| \leq M\}}\right]=\mathbb{E}\left[Y^{2} \mathbb{1}_{\{|Y|>M\}}\right]+\mathbb{E}\left[\left(Y^{+}\right)^{2} \mathbb{1}_{\{|Y| \leq M\}}\right]$, because $Y^{+}=Y$ on the event $\{|Y|>M\}$. For the second term, since $0 \leq Y^{+} \leq M$ on the event $\{|Y| \leq M\}$, we can write $\left(Y^{+}\right)^{2} \leq M Y^{+}$(no absolute value needed). Since $Y^{-}=Y^{-} \mathbb{1}_{\{|Y| \leq M\}}$ has zero mean by (4.16), we obtain

$$
\begin{aligned}
\mathbb{E}\left[\left(Y^{+}\right)^{2} \mathbb{1}_{\{|Y| \leq M\}}\right] & \leq M \mathbb{E}\left[Y^{+} \mathbb{1}_{\{|Y| \leq M\}}\right]=M \mathbb{E}\left[\left(Y^{+}+Y^{-}\right) \mathbb{1}_{\{|Y| \leq M\}}\right] \\
& =M \mathbb{E}\left[Y \mathbb{1}_{\{|Y| \leq M\}}\right]=M\left(-\mathbb{E}\left[Y \mathbb{1}_{\{|Y|>M\}}\right]\right) \leq \mathbb{E}\left[Y^{2} \mathbb{1}_{\{|Y|>M\}}\right],
\end{aligned}
$$

where we have used the fact that $\mathbb{E}[Y]=0$ by assumption, and Markov's inequality. The last relation in (4.16) is proved.

Step 5. We apply the decomposition (4.15) to the random variables $\left(X_{i}^{j}\right)_{i \in[N]}$, defined in (4.9), where the extra randomness used in the construction is taken independently for each variable: then we write

$$
X_{i}^{j}=X_{i}^{j-}+X_{i}^{j+},
$$

and the properties in (4.16) are satisfied. Note that by (4.13) we can write

$$
L_{j}\left(X^{j}\right)-L_{j}\left(X^{j-}\right)=\sum_{I \ni j,|I| \leq \ell} \psi(I) \sum_{\Gamma \subseteq I,|\Gamma| \geq 1}\left(X^{j+}\right)^{\Gamma}\left(X^{j-}\right)^{I \backslash \Gamma} .
$$

Since the random variables $X_{1}^{j-}, X_{1}^{j+}, X_{2}^{j-}, X_{2}^{j+}, \ldots$ are orthogonal in $L^{2}$ by construction, setting $\sigma_{ \pm, i}^{2}:=\mathbb{E}\left[\left(X_{i}^{j \pm}\right)^{2}\right]$ and observing that $\sigma_{-, i}^{2}+\sigma_{+, i}^{2}=\operatorname{Var}\left(X_{i}^{j}\right)=1$, we obtain

$$
\begin{aligned}
\mathbb{E}\left[\left(L_{j}\left(X^{j}\right)-L_{j}\left(X^{j-}\right)\right)^{2}\right] & =\sum_{I \ni j,|I| \leq \ell} \psi(I)^{2} \sum_{\Gamma \subseteq I,|\Gamma| \geq 1}\left(\sigma_{+}^{2}\right)^{\Gamma}\left(\sigma_{-}^{2}\right)^{I \backslash \Gamma} \\
& =\sum_{I \ni j,|I| \leq \ell} \psi(I)^{2}\left(1-\left(\sigma_{-}^{2}\right)^{I}\right) \leq \sum_{I \ni j,|I| \leq \ell} \psi(I)^{2}\left(1-\left(1-\bar{\sigma}_{+}^{2}\right)^{|I|}\right),
\end{aligned}
$$

where

$$
\bar{\sigma}_{+}^{2}:=\max _{i=1, \ldots, N} \sigma_{+, i}^{2}=\max _{i=1, \ldots, N} \mathbb{E}\left[\left(X_{i}^{j+}\right)^{2}\right] \leq 2 \max _{i=1, \ldots, N} \mathbb{E}\left[\left(X_{i}^{j}\right)^{2} \mathbb{1}_{\left\{\left|X_{i}^{j}\right|>M\right\}}\right] \leq 2 m_{2}^{>M},
$$

having used (4.16) and the definition of $m_{2}^{>M}$ in (2.22) (recall (4.9) ). Since $1-(1-p)^{n} \leq n p$, we obtain

$$
\begin{aligned}
\sum_{j=1}^{N} \mathbb{E}\left[\left(L_{j}\left(X^{j}\right)-L_{j}\left(X^{j-}\right)\right)^{2}\right] & \leq 2 m_{2}^{>M} \sum_{j=1}^{N}\left(\sum_{I \ni j,|I| \leq \ell}|I| \psi(I)^{2}\right) \\
& \leq 2 m_{2}^{>M} \ell^{2} \sum_{|I| \leq \ell} \psi(I)^{2} .
\end{aligned}
$$

Tracing back through (4.7), (4.11), (4.12) and (4.14), we note that this gives the first term in the right hand side of (2.24). 
Step 6. We finally consider the contribution of the second term in (4.14). We apply the hypercontractivity results in [MOO10]: by Propositions 3.16 and 3.12 therein, denoting by $\|Y\|_{q}:=\mathbb{E}\left[|Y|^{q}\right]^{1 / q}$ the usual $L^{q}$ norm, for every $q>2$ we have

$$
\left\|L_{j}\left(X^{j-}\right)\right\|_{q} \leq\left(B_{q}\right)^{\ell}\left\|L_{j}\left(X^{j-}\right)\right\|_{2}
$$

where

$$
B_{q}:=2 \sqrt{q-1} \max _{i \in[N]} \frac{\left\|X_{i}^{j-}\right\|_{q}}{\left\|X_{i}^{j-}\right\|_{2}} .
$$

Let us set $Y:=X_{i}^{j}$ for short and choose $q=3$. Since $\left|Y^{-}\right| \leq M$, by (4.16), we have

$$
\left\|Y^{-}\right\|_{3} \leq \mathbb{E}\left[|Y|^{3} \mathbb{1}_{\{|Y| \leq M\}}\right]^{1 / 3} \leq\left(m_{3}^{\leq M}\right)^{1 / 3},
$$

where we recall (4.9) and the definition of $m_{3}^{\leq M}$ from (2.22). On the other hand, again by (4.16),

$$
\begin{aligned}
\left\|Y^{-}\right\|_{2}^{2} & =\|Y\|_{2}^{2}-\left\|Y^{+}\right\|_{2}^{2}=\mathbb{E}\left[Y^{2}\right]-\mathbb{E}\left[\left(Y^{+}\right)^{2}\right] \geq \mathbb{E}\left[Y^{2}\right]-2 \mathbb{E}\left[Y^{2} \mathbb{1}_{\{|Y|>M\}}\right] \\
& =1-2 \mathbb{E}\left[\left(X_{i}^{j}\right)^{2} \mathbb{1}_{\left\{\left|X_{i}^{j}\right|>M\right\}}\right] \geq 1-2 m_{2}^{>M}
\end{aligned}
$$

hence

$$
B_{3} \leq 2 \sqrt{2} \frac{\left(m_{3}^{\leq M}\right)^{1 / 3}}{\sqrt{1-2 m_{2}^{>M}}} \leq 4\left(m_{3}^{\leq M}\right)^{1 / 3},
$$

provided $m_{>M}^{(2)} \leq \frac{1}{4}$, as in the assumptions of Theorem 2.6. Therefore, (4.17) for $q=3$ yields

$$
\mathbb{E}\left[\left|L_{j}\left(X^{j-}\right)\right|^{3}\right] \leq 64^{\ell}\left(m_{3}^{\leq M}\right)^{\ell} \mathbb{E}\left[L_{j}\left(X^{j-}\right)^{2}\right]^{3 / 2} .
$$

Note that, since $\mathbb{E}\left[\left(X_{i}^{j-}\right)^{2}\right] \leq \mathbb{E}\left[\left(X_{i}^{j}\right)^{2}\right]=1$, we have

$$
\mathbb{E}\left[L_{j}\left(X^{j-}\right)^{2}\right]=\sum_{I \ni j,|I| \leq \ell} \psi(I)^{2} \prod_{i \in I} \mathbb{E}\left[\left(X_{i}^{j-}\right)^{2}\right] \leq \sum_{I \ni j,|I| \leq \ell} \psi(I)^{2}=\operatorname{Inf}_{j}\left[\Psi^{\leq \ell}\right] .
$$

Therefore

$$
\begin{aligned}
\sum_{j=1}^{N} \mathbb{E}\left[\left|L_{j}\left(X^{j-}\right)\right|^{3}\right] & \leq 64^{\ell}\left(m_{3}^{\leq M}\right)^{\ell}\left(\max _{i \in[N]} \sqrt{\operatorname{Inf}_{i}[\Psi \leq \ell]}\right) \sum_{j=1}^{N} \sum_{I \ni j,|I| \leq \ell} \psi(I)^{2} \\
& \leq \ell 64^{\ell}\left(m_{3}^{\leq M}\right)^{\ell}\left(\max _{i \in[N]} \sqrt{\operatorname{Inf}_{i}[\Psi \leq \ell]}\right) \sum_{|I| \leq \ell} \psi(I)^{2} .
\end{aligned}
$$

This gives the third term in the right hand side of (2.24) (because $\frac{8}{3} \ell 64^{\ell} \leq 70^{\ell+1}$ ), which completes the proof.

\section{Proof of the CONVERgence to Wiener ChaOS}

In this section, we prove Theorems 2.3 and 2.5 on the convergence of polynomial chaos expansions to Wiener chaos expansions.

Proof of Theorem 2.3. Let $W(\cdot)$ be the $d$-dimensional white noise used to define the Wiener chaos expansion for $\boldsymbol{\Psi}_{0}$ in Theorem 2.3. Given the tessellation $\mathcal{C}_{\delta}$ indexed by $\mathbb{T}_{\delta}$, where each cell $\mathcal{C}_{\delta}(x)$ has the same volume $v_{\delta}$, for each $x \in \mathbb{T}_{\delta}$, we define

$$
\xi_{\delta, x}:=\mu_{\delta}(x)+v_{\delta}^{-1 / 2} \int_{\mathcal{C}_{\delta}(x)} \sigma_{\delta} W(\mathrm{~d} y)=v_{\delta}^{-1 / 2} \int_{\mathcal{C}_{\delta}(x)}\left(\sigma_{\delta} W(\mathrm{~d} y)+\bar{\mu}_{\delta}(y) \mathrm{d} y\right),
$$


where we recall that $\bar{\mu}_{\delta}:=v_{\delta}^{-1 / 2} \mu_{\delta}$ by (2.9). Note that $\xi_{\delta}:=\left(\xi_{\delta, x}\right)_{x \in \mathbb{T}}$ is a family of independent Gaussian random variables with the same mean and variance as $\zeta_{\delta}=\left(\zeta_{\delta, x}\right)_{x \in \mathbb{T}_{\delta}}$.

We recall that our goal is to show that $\Psi_{\delta}\left(\zeta_{\delta}\right) \rightarrow \boldsymbol{\Psi}_{0}$ in distribution. The strategy is first to focus on $\Psi_{\delta}\left(\xi_{\delta}\right)$ instead of $\Psi_{\delta}\left(\zeta_{\delta}\right)$ : we can write $\Psi_{\delta}\left(\xi_{\delta}\right)$ as a Wiener chaos expansion with respect to $W(\cdot)$, like $\mathbf{\Psi}_{0}$, and show that $\mathbb{E}\left[\left|\Psi_{\delta}\left(\xi_{\delta}\right)-\mathbf{\Psi}_{0}\right|^{2}\right] \rightarrow 0$ as $\delta \downarrow 0$; then we use the Lindeberg principle, Theorem 2.8, to replace $\Psi_{\delta}\left(\xi_{\delta}\right)$ by $\Psi_{\delta}\left(\zeta_{\delta}\right)$.

Step 1. We first show that for each degree $k \in \mathbb{N}_{0}$,

$$
\lim _{\delta \downarrow 0} \mathbb{E}\left[\left|\Psi_{\delta}^{(k)}\left(\xi_{\delta}\right)-\Psi_{0}^{(k)}\right|^{2}\right]=0,
$$

where $\Psi_{\delta}^{(k)}$ is a polynomial with kernel $\psi_{\delta}^{(k)}(I):=\psi_{\delta}(I) \mathbb{1}_{\{|I|=k\}}$, and similarly, $\Psi_{0}^{(k)}$ is defined as $\boldsymbol{\Psi}_{0}$ in (2.12), except the kernel $\boldsymbol{\psi}_{0}$ therein is replaced by $\boldsymbol{\psi}_{0}^{(k)}(I):=\boldsymbol{\psi}_{0}(I) \mathbb{1}_{\{|I|=k\}}$ (that is, we take the $k$-th term in the sum). Recalling from (2.10) that $\bar{\psi}_{\delta}(I):=v_{\delta}^{-|I| / 2} \psi_{\delta}(I)$, and extending $\bar{\psi}_{\delta}$ to a function defined on $\mathbb{R}^{k}$, as discussed before Theorem 2.3, we can write

$$
\begin{aligned}
\Psi_{\delta}^{(k)}\left(\xi_{\delta}\right) & =\sum_{I \in \mathcal{P}^{\mathrm{fin}}\left(\mathbb{T}_{\delta}\right),|I|=k} \psi_{\delta}(I) \xi_{\delta}^{I}=\frac{1}{k !} \int \cdots \int_{\left(\mathbb{R}^{d}\right)^{k}} \bar{\psi}_{\delta}\left(y_{1}, \ldots, y_{k}\right) \prod_{i=1}^{k}\left(\sigma_{\delta} W\left(\mathrm{~d} y_{i}\right)+\bar{\mu}_{\delta}\left(y_{i}\right) \mathrm{d} y_{i}\right) \\
& =\frac{1}{k !} \sum_{I \subset[k]:=\{1, \ldots, k\}} \int_{\left(\mathbb{R}^{d}\right)^{k-|I|}}\left(\int_{\left(\mathbb{R}^{d}\right)^{|I|}} \sigma_{\delta}^{k-|I|} \bar{\psi}_{\delta}\left(y_{1}, \ldots, y_{k}\right) \prod_{i \in I} \bar{\mu}_{\delta}\left(y_{i}\right) \mathrm{d} y_{i}\right) \prod_{j \in[k] \backslash I} W\left(\mathrm{~d} y_{j}\right) .
\end{aligned}
$$

A similar expansion holds for $\boldsymbol{\Psi}_{0}^{(k)}$ with $\bar{\psi}_{\delta}, \bar{\mu}_{\delta}, \sigma_{\delta}$ replaced respectively by $\boldsymbol{\psi}_{0}, \boldsymbol{\mu}_{0}, \boldsymbol{\sigma}_{0}$. Comparing the two expansions term by term for each $I \subset[k]$, we then obtain, by the triangle inequality and the Ito isometry (2.2),

$$
\begin{aligned}
& \mathbb{E}\left[\left|\Psi_{\delta}^{(k)}\left(\xi_{\delta}\right)-\Psi_{0}^{(k)}\right|^{2}\right]^{\frac{1}{2}} \\
\leq & \frac{1}{k !} \sum_{I \subset[k]}\left(\int_{\left(\mathbb{R}^{d}\right)^{k-|I|}}\left\{\int_{\left(\mathbb{R}^{d}\right)^{|I|}}\left(\sigma_{\delta}^{k-|I|} \bar{\psi}_{\delta} \prod_{i \in I} \bar{\mu}_{\delta}\left(y_{i}\right)-\sigma_{0}^{k-|I|} \psi_{0} \prod_{i \in I} \boldsymbol{\mu}_{0}\left(y_{i}\right)\right) \prod_{i \in I} \mathrm{~d} y_{i}\right\}^{2} \prod_{j \in[k] \backslash I} \mathrm{~d} y_{j}\right)^{\frac{1}{2}} .
\end{aligned}
$$

To see that each term above tends to 0 as $\delta \downarrow 0$, let us assume w.l.o.g. that either $I=\emptyset$ or $I=[n]$ for some $1 \leq n \leq k$. We then write in a telescopic sum

$$
\begin{aligned}
& \sigma_{\delta}^{k-n} \bar{\psi}_{\delta} \prod_{i=1}^{n} \bar{\mu}_{\delta}\left(y_{i}\right)-\boldsymbol{\sigma}_{0}^{k-n} \boldsymbol{\psi}_{0} \prod_{i=1}^{n} \boldsymbol{\mu}_{0}\left(y_{i}\right) \\
= & \Delta_{0} \prod_{i=1}^{n} \bar{\mu}_{\delta}\left(y_{i}\right)+\boldsymbol{\sigma}_{0}^{k-n} \boldsymbol{\psi}_{0} \Delta_{1} \prod_{i=2}^{n} \bar{\mu}_{\delta}\left(y_{i}\right)+\cdots+\boldsymbol{\sigma}_{0}^{k-n} \boldsymbol{\psi}_{0} \prod_{i=1}^{n-1} \boldsymbol{\mu}_{0}\left(y_{i}\right) \Delta_{n},
\end{aligned}
$$

where $\Delta_{0}:=\boldsymbol{\sigma}_{\delta}^{k-n} \bar{\psi}_{\delta}-\boldsymbol{\sigma}_{0}^{k-n} \boldsymbol{\psi}_{0}$ and $\Delta_{i}:=\bar{\mu}_{\delta}\left(y_{i}\right)-\boldsymbol{\mu}_{0}\left(y_{i}\right)$ for $i=1, \ldots, n$. The contribution of each term from (5.4) to the integrals in (5.3) can be bounded by applying Cauchy-Schwarz to the inner integral, in such a way that the $\psi$ term is separated from the product of the $\mu$ 's. It is then easily seen that all terms tend to 0 as $\delta \downarrow 0$ by the assumptions in Theorem 2.3 that $\bar{\mu}_{\delta} \rightarrow \boldsymbol{\mu}_{0}$ and $\bar{\psi}_{\delta} \rightarrow \boldsymbol{\psi}_{0}$ in $L^{2}$, together with $\sigma_{\delta} \rightarrow \boldsymbol{\sigma}_{0} \in(0, \infty)$. This implies (5.2).

Step 2. We next give a uniform $L^{2}$ bound on the tail of the series for $\Psi_{\delta}\left(\zeta_{\delta}\right)$ and $\boldsymbol{\Psi}_{0}$. More precisely, for any $\ell<N$, let $\Psi_{\delta}^{(\ell, N)}:=\sum_{\ell<k<N} \Psi_{\delta}^{(k)}$ and $\Psi_{0}^{(\ell, N)}:=\sum_{\ell<k<N} \Psi_{0}^{(k)}$. Denote 
$\Psi_{\delta}^{>\ell}$ and $\Psi_{0}^{>\ell}$ for the case $N=\infty$. We will show that

$$
\lim _{\ell \rightarrow \infty} \limsup _{\delta \downarrow 0} \mathbb{E}\left[\left|\Psi_{\delta}^{>\ell}\left(\zeta_{\delta}\right)\right|^{2}\right]=0, \quad \text { and } \quad \Psi_{0}=\sum_{k=0}^{\infty} \Psi_{0}^{(k)} \quad \text { converges in } L^{2} .
$$

Together with (5.2) and the fact that $\mathbb{E}\left[\Psi_{\delta}^{(\ell, N)}\left(\xi_{\delta}\right)^{2}\right]=\mathbb{E}\left[\Psi_{\delta}^{(\ell, N)}\left(\zeta_{\delta}\right)^{2}\right]$ for all $0 \leq \ell<N \leq \infty$, it follows that

$$
\mathbb{E}\left[\Psi_{\delta}\left(\zeta_{\delta}\right)^{2}\right]=\mathbb{E}\left[\Psi_{\delta}\left(\xi_{\delta}\right)^{2}\right] \rightarrow \mathbb{E}\left[\Psi_{0}^{2}\right] \quad \text { as } \delta \downarrow 0,
$$

which is one of the claims in Theorem 2.3

If we denote $\xi_{\delta, x}=\mu_{\delta}(x)+\widetilde{\xi}_{\delta, x}$ and let $\widetilde{\Psi}_{\delta}^{(\ell, N)}(x):=\Psi_{\delta}^{(\ell, N)}\left(x+\mu_{\delta}\right)$, then for $\varepsilon>0$ as specified in Theorem 2.3 (iii) we can apply Lemma 4.1 (actually a modification of it, where we take into account that the random variables do not have normalized variance) to obtain

$$
\mathbb{E}\left[\left|\Psi_{\delta}^{(\ell, N)}\left(\xi_{\delta}\right)\right|^{2}\right]=\mathbb{E}\left[\left|\widetilde{\Psi}_{\delta}^{(\ell, N)}\left(\widetilde{\xi}_{\delta}\right)\right|^{2}\right] \leq e^{\frac{1}{\varepsilon \sigma_{\delta}^{2}} \sum_{x \in \mathbb{T}_{\delta}} \mu_{\delta}(x)^{2}} \sum_{\substack{I \in \mathcal{P} \text { fin } \\ \ell<|I|<N}}\left(1+\varepsilon \mathbb{T}_{\delta}\right)\left(\sigma_{\delta}\right)^{|I|} \psi_{\delta}(I)^{2} .
$$

Similar relation as (5.6) holds for $\zeta_{\delta}$ replacing $\xi_{\delta}$. Since $\sum_{x \in \mathbb{T}_{\delta}} \mu_{\delta}(x)^{2}=\left\|\bar{\mu}_{\delta}\right\|_{L^{2}\left(\mathbb{R}^{d}\right)}^{2}($ recall the extension of $f: \mathbb{T}_{\delta} \rightarrow \mathbb{R}$ to $f: \mathbb{R}^{d} \rightarrow \mathbb{R}$ as specified before Theorem 2.3), the assumptions in Theorem 2.3 (ii) and (iii) immediately imply the first limit in (5.5) if we let $N \uparrow \infty$ in (5.6). It also shows that $\Psi_{\delta}\left(\xi_{\delta}\right)=\sum_{k=0}^{\infty} \Psi_{\delta}^{(k)}\left(\xi_{\delta}\right)$, as well as $\Psi_{\delta}\left(\zeta_{\delta}\right)$, are $L^{2}$ convergent series.

By (5.2), we can take the limit $\delta \downarrow 0$ in (5.6) to obtain

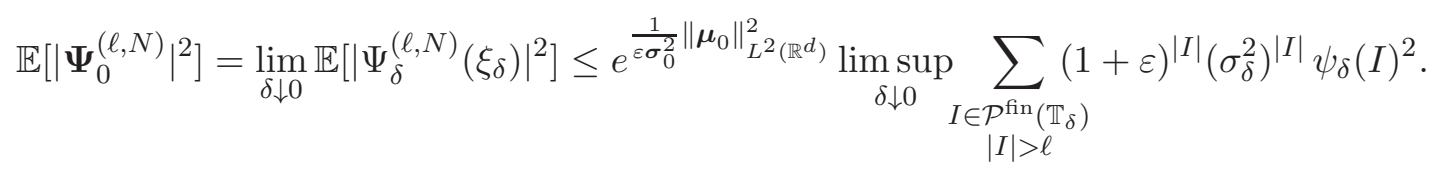

By assumptions (ii) and (iii) of Theorem 2.3, this implies that $\boldsymbol{\Psi}_{0}=\sum_{k=0}^{\infty} \mathbf{\Psi}_{0}^{(k)}$ is an $L^{2}$ convergent series, proving the second relation in (5.5).

We also observe that if $\mu_{\delta}(x) \equiv 0$ there is no need to apply Lemma 4.1, relation (5.6) holds without the exponential pre-factor and with $\varepsilon=0$, because $\xi_{\delta}(x), x \in \mathbb{T}_{\delta}$ are random variables with zero mean and finite variance $\sigma_{\delta}$ (cf. Remark 5.5).

Step 3. We now use the Lindeberg principle, Theorem 2.8, to show that for each $\ell \in \mathbb{N}_{0}$, $\Psi_{\delta}^{\leq \ell}\left(\zeta_{\delta}\right):=\sum_{k=0}^{\ell} \Psi_{\delta}^{(k)}\left(\zeta_{\delta}\right)$ has the same limiting distribution as $\Psi_{\delta}^{\leq \ell}\left(\xi_{\delta}\right)$ as $\delta \downarrow 0$. Together with the $L^{2}$ convergence of $\Psi_{\delta}^{\leq \ell}\left(\xi_{\delta}\right)$ to $\Psi_{0}^{\leq \ell}:=\sum_{k=0}^{\ell} \Psi_{0}^{(k)}$ proved in Step 1, cf. (5.2), as well as the uniform $L^{2}$ bound on $\Psi_{\delta}^{>\ell}\left(\zeta_{\delta}\right)$ and $\Psi_{0}^{>\ell}$ shown in Step 2, cf. (5.5), this implies that $\Psi_{\delta}\left(\zeta_{\delta}\right)$ converges in distribution to $\boldsymbol{\Psi}_{0}$ as $\delta \downarrow 0$.

It suffices to show that for all $f \in \mathscr{C}^{3}$ with $C_{f}:=\max \left\{\left\|f^{\prime}\right\|_{\infty},\left\|f^{\prime \prime}\right\|_{\infty},\left\|f^{\prime \prime \prime}\right\|_{\infty}\right\}<\infty$,

$$
\lim _{\delta \downarrow 0}\left|\mathbb{E}\left[f\left(\Psi_{\delta}^{\leq \ell}\left(\zeta_{\delta}\right)\right)-f\left(\Psi_{\delta}^{\leq \ell}\left(\xi_{\delta}\right)\right)\right]\right|=0 .
$$

With $\varepsilon$ as specified in Theorem 2.3 (iii), we can apply Theorem 2.8 (actually a slight modification of it, taking into account the non normalized variance $\sigma_{\delta}$ of the variables used here): the absolute value in the left hand side of (5.7) is bounded by

$$
e^{\frac{2}{\varepsilon \sigma_{\delta}^{2}}\left\|\bar{\mu}_{\delta}\right\|_{L^{2}\left(\mathbb{R}^{d}\right)}^{2}} C_{f} \widehat{C}_{\Psi_{\delta}^{(\varepsilon), \leq \ell}}\left(16 \ell^{2} \frac{m_{2}^{>M}}{\sigma_{\delta}^{2}}+70^{\ell+1}\left(\frac{m_{3}^{\leq M}}{\sigma_{\delta}^{3}}\right)^{\ell} \max _{i \in \mathbb{T}_{\delta}} \sqrt{\widehat{\operatorname{Inf}}_{i}\left[\Psi_{\delta}^{(\varepsilon), \leq \ell}\right]}\right),
$$


where

$$
\begin{gathered}
\widehat{\mathcal{C}}_{\Psi_{\delta}^{(\varepsilon), \leq \ell}}:=\sum_{|I| \leq \ell}(1+\varepsilon)^{|I|}\left(\sigma_{\delta}^{2}\right)^{|I|} \psi_{\delta}(I)^{2}=\sum_{k=0}^{\ell}(1+\varepsilon)^{k} \frac{1}{k !}\left\|\sigma_{\delta} \bar{\psi}_{\delta}\right\|_{L^{2}\left(\left(\mathbb{R}^{d}\right)^{k}\right)}^{2} \\
\widehat{\operatorname{Inf}}_{i}\left[\Psi_{\delta}^{(\varepsilon), \leq \ell}\right]:=\sum_{I: I \ni i,|I| \leq \ell}(1+\varepsilon)^{|I|}\left(\sigma_{\delta}^{2}\right)^{|I|} \psi_{\delta}(I)^{2}=\sum_{k=1}^{\ell} \frac{(1+\varepsilon)^{k}}{(k-1) !}\left(\sigma_{\delta}^{2}\right)^{k}\left\|\bar{\psi}_{\delta} \mathbb{1}_{\left\{x_{1} \in \mathcal{C}_{\delta}(i)\right\}}\right\|_{L^{2}\left(\left(\mathbb{R}^{d}\right)^{k}\right)}^{2},
\end{gathered}
$$

and we recall that $\mathcal{C}_{\delta}(i) \subset \mathbb{R}^{d}$ is the cell indexed by $i \in \mathbb{T}_{\delta}$ in the tessellation $\mathcal{C}_{\delta}$. We are left with showing that (5.8) vanishes as $\delta \downarrow 0$. Note that

- $\widehat{\mathrm{C}}_{\Psi_{\delta}^{(\varepsilon), \leq \ell}}$ is uniformly bounded by Theorem 2.3 (iii)-(iii);

- $\left\|\bar{\mu}_{\delta}\right\|_{L^{2}\left(\mathbb{R}^{d}\right)}^{2} \rightarrow\left\|\boldsymbol{\mu}_{0}\right\|_{L^{2}\left(\mathbb{R}^{d}\right)}^{2}$ and $\sigma_{\delta} \rightarrow \boldsymbol{\sigma}_{0}>0$ by Theorem 2.3 (il);

- $m_{3}^{\leq M} \leq M^{3}$ and $m_{2}^{>M}$ can be made arbitrarily small by choosing $M$ large, by the definition (2.22) (to be applied to the centered variables $\zeta_{\delta, x}-\mathbb{E}\left[\zeta_{\delta, x}\right]$ ) and the fact that $\left(\left(\zeta_{\delta, x}-\mathbb{E}\left[\zeta_{\delta, x}\right]\right)^{2}\right)_{\delta \in(0,1), x \in \mathbb{T}_{\delta}}$ are uniformly integrable by assumption.

It only remains to verify that $\widehat{\operatorname{Inf}}_{i}\left[\Psi_{\delta}^{(\varepsilon), \leq \ell}\right]$ vanishes as $\delta \downarrow 0$, uniformly in $i \in \mathbb{T}_{\delta}$. Since

$$
\widehat{\operatorname{Inf}}_{i}\left[\Psi_{\delta}^{(\varepsilon), \leq \ell}\right] \leq 2 \sum_{k=1}^{\ell} \frac{(1+\varepsilon)^{k}}{(k-1) !}\left(\sigma_{\delta}^{2}\right)^{k}\left(\left\|\bar{\psi}_{\delta}-\boldsymbol{\psi}_{0}\right\|_{L^{2}\left(\left(\mathbb{R}^{d}\right)^{k}\right)}^{2}+\left\|\boldsymbol{\psi}_{0} \mathbb{1}_{\left\{x_{1} \in \mathcal{C}_{\delta}(i)\right\}}\right\|_{L^{2}\left(\left(\mathbb{R}^{d}\right)^{k}\right)}^{2}\right)
$$

one has $\left\|\bar{\psi}_{\delta}-\boldsymbol{\psi}_{0}\right\|_{L^{2}\left(\left(\mathbb{R}^{d}\right)^{k}\right)}^{2} \rightarrow 0$ by Theorem 2.3 (iii), and $\left\|\boldsymbol{\psi}_{0} \mathbb{1}_{\left\{x_{1} \in \mathcal{C}(i)\right\}}\right\|_{L^{2}\left(\left(\mathbb{R}^{d}\right)^{k}\right)}^{2} \rightarrow 0$ uniformly in $i \in \mathbb{T}_{\delta}$ because $\operatorname{Leb}\left(\mathcal{C}_{\delta}(i)\right)=v_{\delta} \downarrow 0$ uniformly in $i \in \mathbb{T}_{\delta}$. This completes Step 3 and establishes the convergence of $\Psi_{\delta}\left(\zeta_{\delta}\right)$ to $\Psi_{0}$ in distribution.

Step 4. Lastly, to show that the convergence of $\Psi_{\delta}\left(\zeta_{\delta}\right)$ to $\Psi_{0}$ in distribution extends to the joint distribution of a finite collection of polynomial chaos expansions $\left(\Psi_{i, \delta}\left(\zeta_{\delta}\right)\right)_{1 \leq i \leq M}$, we note that by the Cramér-Wold device, it suffices to show the convergence of $\sum_{i=1}^{M} c_{i} \Psi_{i, \delta}\left(\zeta_{\delta}\right)$ to $\sum_{i=1}^{M} c_{i} \boldsymbol{\Psi}_{i, 0}$ for any $c_{1}, \ldots, c_{m} \in \mathbb{R}$. This follows from what we have proved so far, since $\sum_{i=1}^{M} c_{i} \Psi_{i, \delta}(x)$ is also a polynomial that satisfies all the required conditions.

Proof of Theorem 2.5. Instead of performing an $L^{2}$ estimate on the tail series $\Psi_{\delta}^{>\ell}\left(\zeta_{\delta}\right)$ as done in Step 2 in the proof of Theorem 2.3, we shall give an $L^{p}$ estimate for any $p \in(0,2)$. More precisely, we replace relation (5.5) by the following one: for any $p \in(0,2)$,

$$
\Psi_{0}=\sum_{k=0}^{\infty} \Psi_{0}^{(k)} \quad \text { converges in } L^{p}, \quad \text { and } \quad \lim _{\ell \rightarrow \infty} \limsup _{\delta \downarrow 0} \mathbb{E}\left[\left|\Psi_{\delta}^{>\ell}\left(\zeta_{\delta}\right)\right|^{p}\right]=0,
$$

and we show that this holds under either condition (a) or (b) in Theorem 2.5. The rest of the proof of Theorem 2.3 then carries over without change.

The key to proving (5.9) is a change of measure argument. For $\ell<N$, let $\Psi_{0}^{(\ell, N)}$ and $\Psi_{\delta}^{(\ell, N)}$ be defined as in Step 2 in the proof of Theorem 2.3. Note that $\Psi_{0}^{(\ell, N)}$ is a finite sum of stochastic integrals with respect to the biased white noise $\boldsymbol{\sigma}_{0} W(\mathrm{~d} x)+\boldsymbol{\mu}_{0}(x) \mathrm{d} x$. By the discussion in Subsection 2.3.1, cf. (2.15), the joint distribution of these stochastic integrals is absolutely continuous with respect to the unbiased case $\boldsymbol{\mu}_{0}(x) \equiv 0$, with Radon-Nikodym 
derivative

$$
\mathrm{f}(W):=\exp \left\{\frac{1}{\boldsymbol{\sigma}_{0}} \int \boldsymbol{\mu}_{0}(y) W(\mathrm{~d} y)-\frac{1}{2 \boldsymbol{\sigma}_{0}^{2}} \int \boldsymbol{\mu}_{0}^{2}(y) \mathrm{d} y\right\} .
$$

Therefore, using $\mathbf{\Psi}_{0, \boldsymbol{\mu}_{0} \equiv 0}^{(\ell, N)}$ to denote $\mathbf{\Psi}_{0}^{(\ell, N)}$ with $\boldsymbol{\mu}_{0}(x) \equiv 0$, for any $p \in(0,2)$ we have

$$
\begin{aligned}
\mathbb{E}\left[\left|\Psi_{0}^{(\ell, N)}\right|^{p}\right] & =\mathbb{E}\left[\mathrm{f}(W)\left|\Psi_{0, \boldsymbol{\mu}_{0} \equiv 0}^{(\ell, N)}\right|^{p}\right] \leq \mathbb{E}\left[\mathrm{f}(W)^{\frac{2}{2-p}}\right]^{\frac{2-p}{2}} \mathbb{E}\left[\left|\Psi_{0, \boldsymbol{\mu}_{0} \equiv 0}^{(\ell, N)}\right|^{2}\right]^{\frac{p}{2}} \\
& =e^{\frac{p}{2(2-p)}\left\|\boldsymbol{\mu}_{0} / \boldsymbol{\sigma}_{0}\right\|_{L^{2}\left(\mathbb{R}^{d}\right)}^{2}} \mathbb{E}\left[\left|\boldsymbol{\Psi}_{0, \boldsymbol{\mu}_{0} \equiv 0}^{(\ell, N)}\right|^{2}\right]^{\frac{p}{2}}
\end{aligned}
$$

by Hölder's inequality. By Theorem 2.3 , when $\mu_{\delta}=\boldsymbol{\mu}_{0} \equiv 0$ it is enough to assume that condition (iii) therein holds with $\varepsilon=0$ to guarantee that $\boldsymbol{\Psi}_{0, \boldsymbol{\mu}_{0} \equiv 0}=\sum_{k=0}^{\infty} \boldsymbol{\Psi}_{0, \boldsymbol{\mu}_{0} \equiv 0}^{(k)}$ is an $L^{2}$ convergent series. Therefore $\boldsymbol{\Psi}_{0}=\sum_{k=0}^{\infty} \mathbf{\Psi}_{0}^{(k)}$ is convergent in $L^{p}$, by (5.11).

To control $\mathbb{E}\left[\left|\Psi_{\delta}^{>\ell}\left(\zeta_{\delta}\right)\right|^{p}\right]$ via a change of measure for $\zeta_{\delta}$ is more subtle, since $\left(\zeta_{\delta, i}\right)_{i \in \mathbb{T}_{\delta}}$ are not assumed to have finite exponential moments. We will instead perform an exponential change of measure on a bounded subset of the support of $\zeta_{\delta, i}$. Since by assumption $\left\|\mu_{\delta}\right\|_{\infty} \rightarrow$ 0 and $\left(\left(\zeta_{\delta, i}-\mu_{\delta}\right)^{2}\right)_{i \in \mathbb{T}_{\delta}}$ are uniformly integrable, also $\left(\zeta_{\delta, i}^{2}\right)_{i \in \mathbb{T}_{\delta}}$ are uniformly integrable. We can then apply Lemma B.1 in Appendix B: there exist independent random variables $\widetilde{\zeta}_{\delta, i}$, whose law is absolutely continuous with respect to the law of $\zeta_{\delta, i}$, with density $f_{\delta, i}(x)$, which satisfy (B.1)-(B.3). We can then write

$$
\begin{aligned}
\mathbb{E}\left[\left|\Psi_{\delta}^{(\ell, N)}\left(\zeta_{\delta}\right)\right|^{p}\right] & =\mathbb{E}\left[\prod_{i \in \mathbb{T}_{\delta}} f_{\delta, i}\left(\zeta_{\delta, i}\right)^{\frac{p}{2}}\left|\Psi_{\delta}^{(\ell, N)}\left(\zeta_{\delta}\right)\right|^{p} \prod_{i \in \mathbb{T}_{\delta}} f_{\delta, i}\left(\zeta_{\delta, i}\right)^{-\frac{p}{2}}\right] \\
& \leq \mathbb{E}\left[\prod_{i \in \mathbb{T}_{\delta}} f_{\delta, i}\left(\zeta_{\delta, i}\right)\left|\Psi_{\delta}^{(\ell, N)}\left(\zeta_{\delta}\right)\right|^{2}\right]^{\frac{p}{2}} \mathbb{E}\left[\prod_{i \in \mathbb{T}_{\delta}} f_{\delta, i}\left(\zeta_{\delta, i}\right)^{-\frac{p}{2-p}}\right]^{\frac{2-p}{2}} \\
& =\mathbb{E}\left[\left|\Psi_{\delta}^{(\ell, N)}\left(\widetilde{\zeta}_{\delta}\right)\right|^{2}\right]^{\frac{p}{2}} \prod_{i \in \mathbb{T}_{\delta}} \mathbb{E}\left[f_{\delta, i}\left(\zeta_{\delta, i}\right)^{-\frac{p}{2-p}}\right]^{\frac{2-p}{2}}
\end{aligned}
$$

Applying (B.1), we have

$$
\prod_{i \in \mathbb{T}_{\delta}} \mathbb{E}\left[f_{\delta, i}\left(\zeta_{\delta, i}\right)^{-\frac{p}{2-p}}\right]^{\frac{2-p}{2}} \leq e^{C_{p} \frac{2-p}{2} \sum_{i \in \mathbb{T}_{\delta}} \mu_{\delta}(i)^{2}}=e^{C_{p} \frac{2-p}{2}\left\|\bar{\mu}_{\delta}\right\|_{L^{2}\left(\mathbb{R}^{2}\right)}^{2},}
$$

which is uniformly bounded for $\delta$ close to 0 (recall that $\bar{\mu}_{\delta} \rightarrow \boldsymbol{\mu}_{0}$ in $L^{2}$ ). To bound the first factor in (5.12), we use the fact that $\left(\widetilde{\zeta}_{\delta, i}\right)_{i \in \mathbb{T}_{\delta}}$ are independent with zero mean to obtain

$$
\mathbb{E}\left[\left|\Psi_{\delta}^{(\ell, N)}\left(\widetilde{\zeta}_{\delta}\right)\right|^{2}\right]=\sum_{\ell<|I|<N}\left(\prod_{i \in I} \mathbb{E}\left[\widetilde{\zeta}_{\delta, i}^{2}\right]\right) \psi_{\delta}(I)^{2} \leq \sum_{\ell<|I|<N} e^{C^{\prime} \sum_{i \in \mathbb{T}_{\delta}} \mu_{\delta}(i)^{2}}\left(\sigma_{\delta}^{2}\right)^{|I|} \psi_{\delta}(I)^{2}
$$

where in the inequality we applied (B.3), provided $\zeta$ satisfies condition (国) in Theorem 2.5 Combined with the assumption in Theorem 2.5 that (2.11) holds with $\varepsilon=0$, and tracing back to (5.12), we then obtain the desired $L^{p}$ bound on $\Psi_{\delta}^{>\ell}\left(\zeta_{\delta}\right)$ in (5.9).

If we assume instead condition (B) in Theorem $[2.5$, then we can modify the calculation in (5.13) by applying the bound $\mathbb{E}\left[\widetilde{\zeta}_{\delta, i}^{2}\right] \leq \sigma_{\delta}^{2}\left(1+C \mu_{\delta}(i)\right)$, stated in (B.1), to obtain

$$
\mathbb{E}\left[\left|\Psi_{\delta}^{(\ell, N)}\left(\widetilde{\zeta}_{\delta}\right)\right|^{2}\right] \leq \sum_{\ell<|I|<N} e^{C\left\|\mu_{\delta}\right\|_{\infty}|I|}\left(\sigma_{\delta}^{2}\right)^{|I|} \psi_{\delta}(I)^{2} .
$$

Theorem 2.5 (b) and condition (2.11) with $\varepsilon=0$ then give the desired bound in (5.9). 


\section{PRoOF FOR DISORDERED PINNING MODEL}

In this section we prove Theorem 3.1. We recall that $\tau=\left(\tau_{k}\right)_{k \geq 0}$ is an aperiodic renewal process such that either $\mathrm{E}\left[\tau_{1}\right]<\infty$, or relation (3.2) holds for some $\alpha \in\left(\frac{1}{2}, 1\right)$. Note that

$$
u(n):=\mathrm{P}(n \in \tau) \sim \begin{cases}\frac{1}{\mathrm{E}\left[\tau_{1}\right]} & \text { if } \mathrm{E}\left[\tau_{1}\right]<\infty \\ \frac{C_{\alpha}}{L(n) n^{1-\alpha}} & \text { if } \frac{1}{2}<\alpha<1 \quad\left(\text { where } C_{\alpha}:=\frac{\alpha \sin (\pi \alpha)}{\pi}\right),\end{cases}
$$

where the first asymptotic relation is the classical renewal theorem, while the second one is due to Doney [D97, Thm. B] (see also [G07, §A.5]). We also recall that $\omega=\left(\omega_{n}\right)_{n \in \mathbb{N}}$, representing the disorder, is an i.i.d. sequence of random variables satisfying (3.1).

Proof of Theorem 3.1. It suffices to rewrite the partition function as a polynomial chaos expansion and then to check that all the conditions of Theorem 2.3 are satisfied. We only consider the conditioned partition function $Z_{N t, \beta_{N}, h_{N}}^{\omega, c}$, as the proof for the free one follows the same lines. We also set $t=1$, to lighten notation.

Step 1. Consider for $N \in \mathbb{N}$ the lattice $\mathbb{T}_{N}:=\frac{1}{N} \mathbb{N}$. Note that in Section 2 we used the notation $\mathbb{T}_{\delta}$ (where $\delta$ would equal $\frac{1}{N}$ ): here we prefer $\mathbb{T}_{N}$, as it indicates the size of the polymer. Consequently, all the quantities in this section will be indexed by $N$ instead of $\delta$. For each $t \in \mathbb{T}_{N}$ we define the cell $\mathcal{C}_{N}(t):=\left(t-\frac{1}{N}, t\right]$, which has volume $v_{N}=\frac{1}{N}$.

Step 2. We now rewrite the conditioned partition function $Z_{N, \beta, h}^{\omega, c}$, cf. (3.4), as a polynomial chaos expansion. This was already done in (1.5)-(1.7), in terms of the random variables $\varepsilon_{i}=e^{\beta \omega_{i}-\Lambda(\beta)+h}-1$. It is actually convenient to rescale the $\varepsilon_{i}$ so that their variance is of order one, in order to apply Theorem 2.3. Since $\operatorname{Var}\left(\varepsilon_{i}\right) \sim \beta^{2}$ as $\beta \downarrow 0$, recalling (3.7) we set

$$
a_{N}=\left\{\begin{array}{ll}
\frac{1}{\sqrt{N}} & \text { if } \quad \mathrm{E}\left[\tau_{1}\right]<\infty \\
\frac{L(N)}{N^{\alpha-1 / 2}} & \text { if } \quad \frac{1}{2}<\alpha<1
\end{array},\right.
$$

so that $\beta_{N}=\hat{\beta} a_{N}$, and we define the random variables $\zeta_{N}=\left(\zeta_{N, t}\right)_{t \in \mathbb{T}_{N}}$ by

$$
\zeta_{N, t}:=\frac{1}{a_{N}}\left(e^{\beta_{N} \omega_{N t}-\Lambda\left(\beta_{N}\right)+h_{N}}-1\right) .
$$

In this way, arguing as in (1.5)-(1.7), we can write

$$
Z_{N, \beta_{N}, h_{N}}^{\omega, c}=\Psi_{N}\left(\zeta_{N}\right):=1+\sum_{k=1}^{N} \frac{1}{k !} \sum_{\left(t_{1}, \ldots, t_{k}\right) \in\left(\mathbb{T}_{N}\right)^{k}} \psi_{N}^{c}\left(t_{1}, \ldots, t_{k}\right) \prod_{i=1}^{k} \zeta_{N, t_{i}},
$$

where the kernel $\psi_{N}^{c}\left(t_{1}, \ldots, t_{k}\right)$ is a symmetric function, which vanishes when $t_{i}=t_{j}$ for some $i \neq j$ or when some $t_{i} \notin(0,1]$, and for $0=: t_{0}<t_{1}<\cdots<t_{k} \leq 1$ is defined by

$$
\begin{aligned}
\psi_{N}^{c}\left(t_{1}, \ldots, t_{k}\right) & :=a_{N}^{k} \mathrm{P}\left(\left\{N t_{1}, \ldots, N t_{k}\right\} \subseteq \tau \mid N \in \tau\right) \\
& =\frac{u\left(N\left(1-t_{k}\right)\right)}{u(N)} \prod_{i=1}^{k} a_{N} u\left(N\left(t_{i}-t_{i-1}\right)\right),
\end{aligned}
$$

recall (6.1). We extend $\psi_{N}^{c}\left(t_{1}, \ldots, t_{k}\right)$ from $\left(\mathbb{T}_{N}\right)^{k}$ to $\mathbb{R}^{k}$ in the usual way, as a piecewise constant function on products of cells. The same is done for $(s, t) \mapsto u(N(t-s))$. 
Step 3. We now verify that the conditions of Theorem 2.3 are satisfied. By our assumptions (3.1) on the disorder, for every fixed $N \in \mathbb{N}$ the random variables $\left(\zeta_{N, t}\right)_{t \in \mathbb{T}_{N}}$ are i.i.d. with mean and variance given by

$$
\begin{aligned}
& \mu_{N}:=\mathbb{E}\left(\zeta_{N, t}\right)=\frac{1}{\sigma_{N}}\left(e^{h_{N}}-1\right) \sim \frac{\hat{h}}{\sqrt{N}}, \\
& \sigma_{N}^{2}:=\operatorname{Var}\left(\zeta_{N, t}\right)=\frac{1}{a_{N}^{2}}\left(e^{\Lambda\left(2 \beta_{N}\right)-2 \Lambda\left(\beta_{N}\right)}-1\right) e^{2 h_{N}} \sim \frac{\beta_{N}^{2}}{a_{N}^{2}} \longrightarrow \hat{\beta}^{2}, \quad \text { as } N \rightarrow \infty .
\end{aligned}
$$

Since $v_{N}=\frac{1}{N}$, condition (1i) of Theorem 2.3 is satisfied with $\boldsymbol{\sigma}_{0}=\hat{\beta}$ and $\boldsymbol{\mu}_{0}(t)=\hat{h} \mathbb{1}_{(0,1]}(t)$. (More precisely, redefining $\zeta_{N, t}$ as $\left(\zeta_{N, t}-\mathbb{E}\left[\zeta_{N, t}\right]\right)$ when $t \notin(0,1]$ - which is harmless, since such values of $t$ do not contribute to (6.4) - one has $\mu_{N}(t)=\mu_{N} \mathbb{1}_{(0,1]}(t) \rightarrow \boldsymbol{\mu}_{0}(t)$ in $L^{2}(\mathbb{R})$.)

To prove that the random variables $\left(\left(\zeta_{N, t}-\mu_{N}\right)^{2}\right)_{N \in \mathbb{N}, t \in \mathbb{T}_{N}}$ are uniformly integrable, we show that the moments $\mathbb{E}\left[\left(\zeta_{N, t}-\mu_{N}\right)^{4}\right]$ are uniformly bounded. Since $\Lambda(\beta)=O(\beta)$ as $\beta \downarrow 0$, by (3.1), for every $k \in \mathbb{N}$ we can estimate

$$
\begin{aligned}
\mathbb{E}\left[\left(e^{\beta \omega-\Lambda(\beta)}-1\right)^{2 k}\right] & \leq 2^{2 k} e^{-2 k \Lambda(\beta)} \mathbb{E}\left[\left(e^{\beta \omega}-1\right)^{2 k}\right]+2^{2 k}\left(1-e^{-\Lambda(\beta)}\right)^{2 k} \\
& =2^{2 k} e^{-2 k \Lambda(\beta)} \mathbb{E}\left[\left(\int_{0}^{\beta} \omega e^{t \omega} \mathrm{d} t\right)^{2 k}\right]+2^{2 k}\left(1-e^{-\Lambda(\beta)}\right)^{2 k} \\
& \leq 2^{2 k} e^{-2 k \Lambda(\beta)} \beta^{2 k} \int_{0}^{\beta} \mathbb{E}\left[\omega^{2 k} e^{2 k t \omega}\right] \frac{\mathrm{d} t}{\beta}+2^{2 k}\left(1-e^{-\Lambda(\beta)}\right)^{2 k}=O\left(\beta^{2 k}\right) .
\end{aligned}
$$

Recalling that $\beta_{N}=\hat{\beta} a_{N}$ and $h_{N}=o(1)$, we obtain the desired bound:

$$
\mathbb{E}\left[\left(\zeta_{N, t}-\mu_{N}\right)^{4}\right] \leq \frac{e^{4 h_{N}}}{a_{N}^{4}} O\left(\beta_{N}^{4}\right)=O(1) .
$$

Let us check condition (iii) of Theorem 2.3. The renewal estimates in (6.1) imply that, for fixed $0<s<t$,

$$
\lim _{N \rightarrow \infty} \sqrt{N} a_{N} u(N(t-s))=\left\{\begin{array}{ll}
\frac{1}{\mathrm{E}\left[\tau_{1}\right]} & \text { if } \mathrm{E}\left[\tau_{1}\right]<\infty \\
\frac{C_{\alpha}}{(t-s)^{1-\alpha}} & \text { if } \frac{1}{2}<\alpha<1
\end{array} .\right.
$$

Recalling the definitions (6.5), (3.9) of the discrete and continuum kernels $\psi_{N}^{c}, \boldsymbol{\psi}_{t}^{c}($ for $t=1$ ), as well as the fact that $v_{N}=\frac{1}{N}$, it follows that for every fixed $k \in \mathbb{N}$ the convergence

$$
v_{N}^{-k / 2} \psi_{N}^{c}\left(t_{1}, \ldots, t_{k}\right) \underset{N \rightarrow \infty}{\longrightarrow} \psi_{1}^{c}\left(t_{1}, \ldots, t_{k}\right)
$$

holds pointwise, for distinct points $t_{1}, \ldots, t_{k}$. To obtain the required $L^{2}$ convergence, it suffices to exhibit an $L^{2}$ domination. The case $\mathrm{E}\left[\tau_{1}\right]<\infty$ is easy: by (6.1) there exists $A \in(0, \infty)$ such that $\frac{1}{A} \leq u(n) \leq A$ for every $n \in \mathbb{N}$, and since $v_{N}^{-1 / 2} a_{N}=1$ it follows that

$$
v_{N}^{-k / 2} \psi_{N}^{c}\left(t_{1}, \ldots, t_{k}\right) \leq A^{k+2} \mathbb{1}_{(0,1]^{k}}\left(t_{1}, \ldots, t_{k}\right) .
$$

We now focus on the case $\frac{1}{2}<\alpha<1$. By Karamata's representation theorem for slowly varying functions BGT87, Theorem 1.3.1], we can write $L(n)=c(n) \exp \left(\int_{1}^{n} \frac{\varepsilon(u)}{u} \mathrm{~d} u\right)$ for 
some functions $c(x) \rightarrow c>0$ and $\varepsilon(x) \rightarrow 0$ as $x \rightarrow \infty$. It follows that for any $\eta>0$ there exits a constant $A^{\prime}=A_{\eta}^{\prime} \in(0, \infty)$ such that

$$
\frac{1}{A^{\prime}}\left(\frac{n}{m}\right)^{-\eta} \leq \frac{L(n)}{L(m)} \leq A^{\prime}\left(\frac{n}{m}\right)^{\eta}, \quad \forall n, m \in \mathbb{N} \text { with } m \leq n .
$$

Recalling (6.1) and (6.2), for possibly a larger constant $A \in(0, \infty)$ we have

$$
\frac{C_{\alpha}}{A(t-s)^{1-\alpha-\eta}} \leq \sqrt{N} a_{N} u(N(t-s)) \leq \frac{A C_{\alpha}}{(t-s)^{1-\alpha+\eta}},
$$

which plugged into (6.5) yields that for $0<t_{1}<\ldots<t_{k} \leq 1$

$$
v_{N}^{-k / 2} \psi_{N}^{c}\left(t_{1}, \ldots, t_{k}\right) \leq \frac{A^{k+2} C_{\alpha}^{k}}{t_{1}^{1-\alpha^{\prime}} \cdots\left(t_{k}-t_{k-1}\right)^{1-\alpha^{\prime}}\left(1-t_{k}\right)^{1-\alpha^{\prime}}},
$$

where we set $\alpha^{\prime}:=\alpha-\eta$ for short. If we choose $\eta>0$ sufficiently small, so that $\alpha^{\prime}>\frac{1}{2}$ (recall that $\alpha>\frac{1}{2}$ ), we have obtained the required $L^{2}$ domination.

We finally check condition (iiii) of Theorem 2.3, that is relation (2.11). Since $\sigma_{N}^{2}$ is bounded, cf. (6.6), we let $B \in(0, \infty)$ denote a constant such that $(1+\varepsilon) \sigma_{N}^{2} \leq B$, so that

$$
\sum_{I \subseteq \mathbb{T}_{N},|I|>\ell}(1+\varepsilon)^{|I|}\left(\sigma_{N}^{2}\right)^{|I|} \psi_{N}^{c}(I)^{2} \leq \sum_{k>\ell} B^{k} \sum_{\substack{\left(t_{1}, \ldots, t_{k}\right) \in\left(\mathbb{T}_{N}\right)^{k} \\ 0<t_{1}<\cdots<t_{k} \leq 1}} \psi_{N}^{c}\left(t_{1}, \ldots, t_{k}\right)^{2} .
$$

If $\mathrm{E}\left[\tau_{1}\right]<\infty$, applying (6.10) and recalling that $v_{N}=\frac{1}{N}$, this expression is bounded by

$$
\sum_{k>\ell} B^{k} A^{2(k+2)}\left(v_{N}\right)^{k} \frac{N^{k}}{k !}=\sum_{k>\ell} \frac{A^{2(k+2)} B^{k}}{k !},
$$

which is arbitrarily small for $\ell$ large, proving (2.11). If $\alpha \in\left(\frac{1}{2}, 1\right)$ we apply (6.12): setting $\chi:=2\left(1-\alpha^{\prime}\right)<1$ for short and bounding the sums by integrals, we estimate (6.13) by

$$
\sum_{k>\ell} B^{k} A^{2(k+2)} C_{\alpha}^{2 k} \int_{0<t_{1}<\cdots<t_{k}<1} \cdots \int_{1} \frac{\mathrm{d} t_{1} \cdots \mathrm{d} t_{k}}{t_{1}^{\chi} \cdots\left(t_{k}-t_{k-1}\right) \chi\left(1-t_{k}\right) \chi} \leq \sum_{k>\ell} B^{k} A^{2(k+2)} C_{\alpha}^{2 k} c_{1} e^{-c_{2} k \log k},
$$

where for the last inequality we have applied Lemma B.3 below (we recall that $\chi<1$ ). Again, the sum can be made arbitrarily small by choosing $\ell$ large, proving (2.11).

Step 4. Lastly, we prove formula (3.11), when $\mathrm{E}\left[\tau_{1}\right]<\infty$. Since $\boldsymbol{\psi}_{t}^{c}\left(t_{1}, \ldots, t_{k}\right)=\left(\frac{1}{\mathrm{E}\left[\tau_{1}\right]}\right)^{k}$ in this case, formula (3.8) for $\hat{h}=0$ yields

$$
\boldsymbol{Z}_{t, \hat{\beta}, 0}^{W, c}=1+\sum_{k=1}^{\infty} \frac{1}{k !}\left(\frac{\hat{\beta}^{k}}{\mathbf{E}\left[\tau_{1}\right]}\right)^{k} \int_{[0, t]^{k}} \ldots \int W\left(\mathrm{~d} t_{1}\right) \cdots W\left(\mathrm{~d} t_{k}\right)=e^{\frac{\hat{\hat{r}}}{\mathbf{E}\left[\tau_{1}\right]} W([0, t])-\frac{1}{2}\left(\frac{\hat{\beta}}{\mathbf{E}\left[\tau_{1}\right]}\right)^{2} t},
$$

where the second equality follows by [J97, Theorem 3.33 and Example 7.12]. This shows that (3.11) holds for $\hat{h}=0$, because $W([0, t]) \sim \mathcal{N}(0, t)$. In the general case, we introduce the tilted law $\widetilde{\mathbb{P}}$ defined by $\mathrm{d} \widetilde{\mathbb{P}} / \mathrm{d} \mathbb{P}=\exp \left\{\left(\frac{\hat{h}}{\hat{\beta}}\right) W([0, t])-\frac{1}{2}\left(\frac{\hat{h}}{\hat{\beta}}\right)^{2} t\right\}$ and note that $\boldsymbol{Z}_{t, \hat{\beta}, \hat{h}}^{W, c}$ under $\mathbb{P}$ has the same law as $\boldsymbol{Z}_{t, \hat{\beta}, 0}^{W, c}$ under $\widetilde{\mathbb{P}}$, cf. (2.15) and (2.16). Since $W([0, t]) \sim \mathcal{N}\left(\frac{\hat{h}}{\hat{\beta}} t, t\right)$ under $\widetilde{\mathbb{P}}$, formula (3.11) is proved also when $\hat{h} \neq 0$. 


\section{PRoof FOR DIRECTED POLYMER MODEL}

In this section we prove Theorem 3.8. We recall that $S=\left(S_{n}\right)_{n \geq 0}$ is a random walk on $\mathbb{Z}$ satisfying Assumption 3.7. We denote by $p \in \mathbb{N}$ the period of the random walk, so that $\mathrm{P}\left(S_{1} \in p \mathbb{Z}+r\right)=1$ for some $r \in\{0, \ldots, p-1\}$. Introducing the lattice

$$
\mathbb{T}:=\left\{(n, k) \in \mathbb{Z}^{2}: k \in p \mathbb{Z}+r n\right\},
$$

we have $\mathrm{P}\left(S=\left(S_{n}\right)_{n \geq 0} \in \mathbb{T}\right)=1$. Defining

$$
q_{n}(k):=\mathrm{P}\left(S_{n}=k\right), \quad \forall n \geq 0, k \in \mathbb{Z},
$$

Gnedenko's local limit theorem [BGT87, Theorem 8.4.1] yields

$$
\sup _{k \in \mathbb{Z}:(n, k) \in \mathbb{T}}\left|n^{1 / \alpha} q_{n}(k)-p g\left(k / n^{1 / \alpha}\right)\right| \underset{n \rightarrow \infty}{\longrightarrow} 0,
$$

where $g(\cdot)$ denotes the density of the stable law to which $S$ is attracted. We also recall that $\omega=(\omega(n, k))_{n \in \mathbb{N}, k \in \mathbb{Z}}$ is an i.i.d. sequence of random variables satisfying (3.1).

Proof of Theorem 3.8. As in the proof of Theorem 3.1 the strategy is to rewrite the partition function as a polynomial chaos expansion and then to apply Theorem 2.3. We focus on the conditioned point-to-point partition function $Z_{N t, \beta_{N}}^{\omega, c}\left(N^{1 / \alpha} x\right)$, as the free one follows the same lines. For notational simplicity, we set $t=1$.

Step 1. We introduce for $N \in \mathbb{N}$ the rescaled lattice

$$
\mathbb{T}_{N}:=\left\{\left(N^{-1} n, N^{-1 / \alpha} k\right):(n, k) \in \mathbb{T}\right\} \subseteq \mathbb{R}^{2},
$$

cf. (7.1). Note that we use $N$ instead of $\delta:=\frac{1}{N}$ as a subscript, as it indicates the "length" of the polymer. For each $(t, x) \in \mathbb{T}_{N}$, we define the cell $\mathcal{C}_{N}((t, x)):=\left(t-\frac{1}{N}, t\right] \times\left(x-\frac{p}{N^{1 / \alpha}}, x\right]$, which has volume $v_{N}=p N^{-(1+1 / \alpha)}$.

Step 2. We rewrite the conditioned partition function $Z_{N, \beta_{N}}^{\omega, c}\left(N^{1 / \alpha} x\right)$, defined in (3.21), as a polynomial chaos expansion, using the random variables $\zeta_{N}=\left(\zeta_{N}(s, y)\right)_{(s, y) \in \mathbb{T}_{N}}$ given by

$$
\zeta_{N}(s, y):=N^{\frac{\alpha-1}{2 \alpha}}\left(e^{\beta_{N} \omega\left(N s, N^{1 / \alpha} y\right)-\Lambda\left(\beta_{N}\right)}-1\right),
$$

where the prefactor has been chosen so that $\operatorname{Var}\left(\zeta_{N}(s, y)\right)=O(1)$, see below. Arguing as in (1.5)-(1.7), we can write

$$
Z_{N, \beta_{N}}^{\omega, c}\left(N^{1 / \alpha} x\right)=\Psi_{N}\left(\zeta_{N}\right):=1+\sum_{k=1}^{N} \frac{1}{k !} \sum_{\left(z_{1}, \ldots, z_{k}\right) \in\left(\mathbb{T}_{N}\right)^{k}} \psi_{N,(1, x)}^{c}\left(z_{1}, \ldots, z_{k}\right) \prod_{i=1}^{k} \zeta_{N}\left(z_{i}\right),
$$

where $\psi_{N,(1, x)}^{c}$ is a symmetric function of $\left(z_{1}, \ldots, z_{k}\right) \in\left(\mathbb{T}_{N}\right)^{k}$, which vanishes when $z_{i}=z_{j}$ for some $i \neq j$ or when some $z_{i} \notin(0,1] \times \mathbb{R}$, and for distinct $z_{1}=\left(t_{1}, x_{1}\right), \ldots, z_{k}=\left(t_{k}, x_{k}\right)$, say with $0<t_{1}<\cdots<t_{k} \leq 1$, is defined by (recall (7.2)

$$
\begin{gathered}
\psi_{N,(1, x)}^{c}\left(\left(t_{1}, x_{1}\right), \ldots,\left(t_{k}, x_{k}\right)\right):=\frac{\mathrm{P}\left(S_{N t_{1}}=N^{1 / \alpha} x_{1}, \ldots, S_{N t_{k}}=N^{1 / \alpha} x_{k} \mid S_{N}=x\right)}{\left(N^{\frac{\alpha-1}{2 \alpha}}\right)^{k}} \\
=\frac{q_{N\left(1-t_{k}\right)}\left(N^{1 / \alpha}\left(x-x_{k}\right)\right)}{q_{N}\left(N^{1 / \alpha} x\right)} \prod_{i=1}^{k}\left(N^{-\frac{\alpha-1}{2 \alpha}} q_{N\left(t_{i}-t_{i-1}\right)}\left(N^{1 / \alpha}\left(x_{i}-x_{i-1}\right)\right)\right),
\end{gathered}
$$

where $\left(t_{0}, x_{0}\right):=(0,0)$. The kernel $\psi_{N,(1, x)}^{c}\left(z_{1}, \ldots, z_{k}\right)$ is extended from $\left(\mathbb{T}_{N}\right)^{k}$ to $\left(\mathbb{R}^{2}\right)^{k}$ in the usual way, as a piecewise constant function which is constant on every product of cells. The same extension is done for the function $((s, x),(t, y)) \mapsto q_{N(t-s)}\left(N^{1 / \alpha}(y-x)\right)$. 
Step 3. We now check the assumptions of Theorem 2.3. Recalling (3.1) and the fact that $\beta_{N}=\hat{\beta} N^{-\frac{\alpha-1}{2 \alpha}}$, cf. Theorem 3.8, for every fixed $N \in \mathbb{N}$ the random variables $\left(\zeta_{N}(z)\right)_{z \in \mathbb{T}_{N}}$ are i.i.d. with zero mean $\mu_{N}(z) \equiv 0$ and variance given by

$$
\sigma_{N}^{2}=\operatorname{Var}\left(\zeta_{N}(z)\right)=N^{\frac{\alpha-1}{\alpha}} e^{\Lambda\left(2 \beta_{N}\right)-2 \Lambda\left(\beta_{N}\right)} \sim N^{\frac{\alpha-1}{\alpha}} \beta_{N}^{2} \underset{N \rightarrow \infty}{\longrightarrow} \hat{\beta}^{2} .
$$

Condition (ii) of Theorem 2.3 is thus satisfied with $\boldsymbol{\mu}_{0}(z) \equiv 0$ and $\boldsymbol{\sigma}_{0}=\hat{\beta}$. The uniform integrability of $\left(\zeta_{N}(z)^{2}\right)_{N \in \mathbb{N}, z \in \mathbb{T}_{N}}$ is easily checked as for the pinning model, cf. (6.7).

Let us check condition (iii). Recalling the definition (3.24) of the function $g_{t}(\cdot)$, we observe that by (7.3), for fixed $0<s<t$ and $x, y \in \mathbb{R}$,

$$
\lim _{N \rightarrow \infty} N^{1 / \alpha} q_{N(t-s)}\left(N^{1 / \alpha}(y-x)\right)=p g_{t-s}(y-x) .
$$

Recalling the definition (3.26) of the continuum kernel $\boldsymbol{\psi}_{t, x}^{c}($ for $t=1)$, since $v_{N}=$ $p N^{-1-1 / \alpha}$, it follows by (7.5) that for every $k \in \mathbb{N}$ one has the pointwise convergence

$$
\lim _{N \rightarrow \infty} v_{N}^{-k / 2} \psi_{N,(1, x)}^{c}\left(\left(t_{1}, x_{1}\right), \ldots,\left(t_{k}, x_{k}\right)\right)=\psi_{1, x}^{c}\left(\left(t_{1}, x_{1}\right), \ldots,\left(t_{k}, x_{k}\right)\right) .
$$

We need to show that this convergence also holds in $L^{2}$. Since the density $g(\cdot)$ is bounded, relation (7.3) yields that for some constant $A \in(0, \infty)$

$$
q_{n}(k) \leq A n^{-1 / \alpha}, \quad \forall n \in \mathbb{N}_{0}, k \in \mathbb{Z} .
$$

For fixed $x \in \mathbb{R}$, one has $q_{N}\left(N^{1 / \alpha} x\right) \geq \frac{p}{2} g(x) / N^{1 / \alpha}$ for large $N$, again by (7.3), hence the prefactor in (7.5) is upper bounded as

$$
\frac{q_{N\left(1-t_{k}\right)}\left(N^{1 / \alpha}\left(x-x_{k}\right)\right)}{q_{N}\left(N^{1 / \alpha} x\right)} \leq \frac{C_{x}}{\left(1-t_{k}\right)^{1 / \alpha}}, \quad \text { where } \quad C_{x}:=\frac{2 A}{(p g(x))} .
$$

Applying (7.8) to each term in the product in (7.5) and recalling that $v_{N}=p N^{-1-1 / \alpha}$, for $0<t_{1}<\ldots<t_{k} \leq 1$ we get

$$
\begin{aligned}
& {\left[v_{N}^{-k / 2} \psi_{N,(1, x)}^{c}\left(\left(t_{1}, x_{1}\right), \ldots,\left(t_{k}, x_{k}\right)\right)\right]^{2} \leq \frac{C_{x}}{\left(1-t_{k}\right)^{1 / \alpha}} \frac{q_{N\left(1-t_{k}\right)}\left(N^{1 / \alpha}\left(x-x_{k}\right)\right)}{q_{N}\left(N^{1 / \alpha} x\right)}} \\
& \quad \times \prod_{i=1}^{k}\left(v_{N}^{-1 / 2} N^{-\frac{\alpha-1}{2 \alpha}}\right)^{2}\left(\frac{A}{N^{1 / \alpha}\left(t_{i}-t_{i-1}\right)^{1 / \alpha}}\right) q_{N\left(t_{i}-t_{i-1}\right)}\left(N^{1 / \alpha}\left(x_{i}-x_{i-1}\right)\right) \\
& =C_{k, x}^{\prime}\left(N^{1 / \alpha}\right)^{k} \frac{\mathrm{P}\left(S_{N t_{1}}=N^{1 / \alpha} x_{1}, \ldots, S_{N t_{k}}=N^{1 / \alpha} x_{k} \mid S_{N}=N^{1 / \alpha} x\right)}{t_{1}^{1 / \alpha}\left(t_{2}-t_{1}\right)^{1 / \alpha} \cdots\left(t_{k}-t_{k-1}\right)^{1 / \alpha}\left(1-t_{k}\right)^{1 / \alpha}}
\end{aligned}
$$

where we set $C_{k, x}^{\prime}:=A^{k} p^{-k} C_{x}$. A further application of (17.8) also yields

$$
\left[v_{N}^{-k / 2} \psi_{N,(1, x)}^{c}\left(\left(t_{1}, x_{1}\right), \ldots,\left(t_{k}, x_{k}\right)\right)\right]^{2} \leq \frac{A^{k} C_{k, x}^{\prime}}{t_{1}^{2 / \alpha}\left(t_{2}-t_{1}\right)^{2 / \alpha} \cdots\left(t_{k}-t_{k-1}\right)^{2 / \alpha}\left(1-t_{k}\right)^{2 / \alpha}} .
$$


We now decompose the domain $\left\{0<t_{1}<\ldots<t_{k}<1\right\} \times \mathbb{R}^{k}$ as $D_{1} \cup D_{2} \cup D_{3}$, where

$$
\begin{gathered}
D_{1}:=\bigcap_{i=1}^{k}\left\{t_{i}-t_{i-1}>\eta,\left|x_{i}\right|<M\right\} \cap\left\{1-t_{k}>\eta\right\}, \\
D_{2}:=\bigcup_{i=1}^{k}\left\{t_{i}-t_{i-1} \leq \eta\right\} \cup\left\{1-t_{k} \leq \eta\right\}, \quad D_{3}:=\bigcup_{i=1}^{k}\left\{\left|x_{i}\right| \geq M\right\},
\end{gathered}
$$

for fixed $\eta, M \in(0, \infty)$. Relation (17.10) shows that the rescaled kernel $v_{N}^{-k / 2} \psi_{N,(1, x)}^{c}(\cdot)$ is uniformly bounded on the (bounded) set $D_{1}$, hence the convergence (7.7) holds in $L^{2}$ on $D_{1}$. If we show that the integrals of $\left[v_{N}^{-k / 2} \psi_{N,(1, x)}^{c}(\cdot)\right]^{2}$ and of $\left[\boldsymbol{\psi}_{1, x}^{c}(\cdot)\right]^{2}$ over the sets $D_{2}$ and $D_{3}$ can be made arbitrarily small, for suitable $\eta, M$, we are done. Since $\boldsymbol{\psi}_{1, x} \in L^{2}\left([0,1]^{k} \times \mathbb{R}^{k}\right)$, by (3.24), there is nothing to prove for $\psi_{1, x}^{c}(\cdot)$ and we may focus on $\psi_{N,(1, x)}^{c}(\cdot)$. By (7.9)

$$
\begin{aligned}
\int_{D_{2}}\left|v_{N}^{-k / 2} \psi_{N,(1, x)}^{c}(\cdot)\right|^{2} & =\sum_{\left.\left(t_{1}, x_{1}\right), \ldots, t_{k}, x_{k}\right) \in\left(\mathbb{T}_{N}\right)^{k}}\left(v_{N}\right)^{k}\left[v_{N}^{-k / 2} \psi_{N,(1, x)}^{c}\left(\left(t_{1}, x_{1}\right), \ldots,\left(t_{k}, x_{k}\right)\right)\right]^{2} \\
& \leq C_{k, x}^{\prime} p^{k} \frac{1}{N^{k}} \sum_{\substack{\left(t_{1}, \ldots, t_{k}\right) \in \frac{1}{N} \mathbb{N} \\
0<t_{1}<\ldots<t_{k} \leq 1 \\
t_{i}-t_{i-1} \leq \eta \text { for some } i}} \frac{1}{t_{1}^{1 / \alpha} \cdots\left(t_{k}-t_{k-1}\right)^{1 / \alpha}\left(1-t_{k}\right)^{1 / \alpha}}, \\
& \leq C_{k, x}^{\prime} p^{k} \int_{\substack{0<t_{1}<\cdots<t_{k}<1 \\
t_{i}-t_{i-1} \leq \eta \text { for some } i}} \frac{\mathrm{d} t_{1} \cdots \mathrm{d} t_{k}}{t_{1}^{1 / \alpha} \cdots\left(t_{k}-t_{k-1}\right)^{1 / \alpha}\left(1-t_{k}\right)^{1 / \alpha}},
\end{aligned}
$$

which vanishes as $\eta \downarrow 0$ (recall that $\alpha>1$ ). Analogously, using again (7.9), we get

$$
\begin{gathered}
\int_{D_{3}}\left|v_{N}^{-k / 2} \psi_{N,(1, x)}^{c}(\cdot)\right|^{2} \leq C_{k, x}^{\prime} p^{k}\left(\int_{0<t_{1}<\cdots<t_{k}<1} \cdots \int_{1} \frac{\mathrm{d} t_{1} \cdots \mathrm{d} t_{k}}{t_{1}^{1 / \alpha} \cdots\left(t_{k}-t_{k-1}\right)^{1 / \alpha}\left(1-t_{k}\right)^{1 / \alpha}}\right) \\
\times \mathrm{P}\left(\max _{0 \leq n \leq N}\left|S_{n}\right| \geq N^{1 / \alpha} M \mid S_{N}=N^{1 / \alpha} x\right) .
\end{gathered}
$$

As $N \rightarrow \infty$, the last probability converges to $\mathrm{P}\left(\sup _{0 \leq t \leq 1}\left|X_{t}\right| \geq M \mid X_{1}=x\right)$, where $X=\left(X_{t}\right)_{t \geq 0}$ is the stable Lévy process to which the random walk is attracted, cf. [Lig68, hence it can be made as small as one wishes, uniformly in $N \in \mathbb{N}$, by choosing $M$ large. This completes the verification of condition (iii) in Theorem 2.3.

Finally, we check condition (iii). Since $\sigma_{N}^{2}$ is bounded, cf. (77.6), we have $(1+\varepsilon) \sigma_{N}^{2} \leq B$ for some $B \in(0, \infty)$ (we can even set $\varepsilon=0$, because $\mu_{N} \equiv 0$ in this case). Applying (7.11) 
for $\eta=1$, i.e. with no restriction on $t_{i}-t_{i-1}$ (equivalently, (7.12) with $M=0$ ), we obtain

$$
\begin{aligned}
& \sum_{I \subset \mathbb{T}_{N},|I|>\ell}(1+\varepsilon)^{|I|}\left(\sigma_{N}^{2}\right)^{|I|} \psi_{N,(1, x)}^{c}(I)^{2} \leq \sum_{k>\ell} B^{k} \frac{1}{k !} \sum_{\left(z_{1}, \ldots, z_{k}\right) \in\left(\mathbb{T}_{N}\right)^{k}} \psi_{N,(1, x)}^{c}\left(z_{1}, \ldots, z_{k}\right)^{2} \\
& \quad=\sum_{k>\ell} B^{k} \frac{1}{k !}\left\|v_{N}^{-k / 2} \psi_{N,(1, x)}^{c}\right\|_{L^{2}\left(\left(\mathbb{R}^{2}\right)^{k}\right)}^{2} \\
& \quad \leq \sum_{k>\ell} \widetilde{B}_{x}^{k} \int_{0<t_{1}<\cdots<t_{k}<1} \cdots \int_{t_{1}^{1 / \alpha} \cdots\left(t_{k}-t_{k-1}\right)^{1 / \alpha}\left(1-t_{k}\right)^{1 / \alpha}} \frac{\mathrm{d} t_{1} \cdots \mathrm{d} t_{k}}{t_{k>\ell}} \widetilde{B}_{x}^{k} c_{1} e^{-c_{2} k \log k}
\end{aligned}
$$

where, recalling that $C_{k, x}^{\prime}=A^{k} p^{-k} C_{x}$, we have set $\widetilde{B}_{x}:=B A \sqrt{p} \max \left\{1, C_{x}\right\}^{2}$, which is a finite constant for every fixed $x \in \mathbb{R}$, and we have applied Lemma B.3 for the last inequality. This shows that (2.11) holds, hence condition (iii) in Theorem 2.3 is verified.

\section{Proof For RANDOM FIELD ISING MODEL}

In this section we prove Theorem 3.14 and Corollary 3.16. We recall that the disordered partition function $Z_{\Omega_{\delta}, \lambda_{\delta}, h_{\delta}}^{+, \omega_{\delta}}$ is defined as in (3.32), where $\Omega_{\delta}:=\Omega \cap(\delta \mathbb{Z})^{2}$ (with $\Omega \subseteq \mathbb{R}^{2}$ being a fixed bounded, simply connected open set with piecewise smooth boundary) and where:

- $\mathrm{P}_{\Omega_{\delta}}^{+}$(with expectation $\mathrm{E}_{\Omega_{\delta}}^{+}$) denotes the critical Ising model on $\mathbb{Z}^{2}$, defined as in (3.30);

- $\omega_{\delta}=\left(\omega_{\delta, x}\right)_{x \in \Omega_{\delta}}$ is an i.i.d. family of random variables satisfying (3.1);

- $\lambda_{\delta}=\left(\lambda_{\delta, x}\right)_{x \in \Omega_{\delta}}, h_{\delta}=\left(h_{\delta, x}\right)_{x \in \Omega_{\delta}}$ are defined by

$$
\lambda_{\delta, x}:=\hat{\lambda}(x) \delta^{\frac{7}{8}}, \quad h_{\delta, x}:=\hat{h}(x) \delta^{\frac{15}{8}},
$$

cf. (3.35), where $\hat{\lambda}: \bar{\Omega} \rightarrow(0, \infty)$ and $\hat{h}: \bar{\Omega} \rightarrow \mathbb{R}$ are fixed continuous functions.

The heart of our proof are pointwise and $L^{2}$ estimates for the critical Ising correlation functions, in particular near the diagonals (see Lemmas 8.18.3 below). Complementary $L^{1}$ estimates have been recently established in [CGN12, Prop.3.9].

Proof of Theorem 3.14. We are going to apply Theorem 2.3, with $v_{\delta}=\delta^{2}$, rewriting the partition function in terms of a polynomial chaos expansion.

Step 1. By relation (3.32), we can write

$$
\begin{gathered}
Z_{\Omega_{\delta}, \lambda_{\delta}, h_{\delta}}^{+, \omega_{\delta}}=\mathrm{E}_{\Omega_{\delta}}^{+}\left[\prod_{x \in \Omega_{\delta}}\left(\cosh \left(\xi_{\delta, x}\right)+\sigma_{x} \sinh \left(\xi_{\delta, x}\right)\right)\right], \\
\text { where } \quad \xi_{\delta, x}:=\lambda_{\delta, x} \omega_{\delta, x}+h_{\delta, x} .
\end{gathered}
$$

Recalling the notation $\alpha^{I}:=\prod_{x \in I} \alpha_{x}$, a binomial expansion of the product yields

$$
\begin{aligned}
e^{-\frac{1}{2}\|\hat{\lambda}\|_{L^{2}(\Omega)}^{2} \delta^{-\frac{1}{4}}} Z_{\Omega_{\delta}, \lambda_{\delta}, h_{\delta}}^{+, \omega_{\delta}} & =e^{-\frac{1}{2}\|\hat{\lambda}\|_{L^{2}(\Omega)}^{2} \delta^{-\frac{1}{4}}} \sum_{I \subseteq \Omega_{\delta}} \cosh \left(\xi_{\delta, .}\right)^{\Omega_{\delta} \backslash I} \mathrm{E}_{\Omega_{\delta}}^{+}\left[\sigma_{.}^{I}\right] \sinh \left(\xi_{\delta, .}\right)^{I} \\
& =e^{-\frac{1}{2}\|\hat{\lambda}\|_{L^{2}(\Omega)}^{2} \delta^{-\frac{1}{4}}} \cosh \left(\xi_{\delta, \cdot}\right)^{\Omega_{\delta}} \sum_{I \subseteq \Omega_{\delta}} \mathrm{E}_{\Omega_{\delta}}^{+}\left[\sigma_{.}^{I}\right] \tanh \left(\xi_{\delta, \cdot}\right)^{I} .
\end{aligned}
$$

We first show that the pre-factor before the sum converges to 1 in probability as $\delta \downarrow 0$. 
Recalling the definition (8.2) of $\xi_{\delta, x}$ and the fact that $\omega_{\delta, x}$ have zero mean, unit variance and locally finite exponential moments, cf. (3.1), a Taylor expansion yields

$$
\mathbb{E}\left[\log \cosh \left(\xi_{\delta, x}\right)\right]=\frac{\lambda_{\delta, x}^{2}}{2}+O\left(h_{\delta, x}^{2}+\lambda_{\delta, x}^{4}\right)=\frac{\hat{\lambda}(x)^{2}}{2} \delta^{\frac{7}{4}}+O\left(\delta^{\frac{7}{2}}\right),
$$

where the term $O\left(\delta^{\frac{7}{2}}\right)$ is uniform over $x \in \Omega_{\delta}$, by the continuity of $\hat{\lambda}, \hat{h}$. Therefore, as $\delta \downarrow 0$,

$$
\sum_{x \in \Omega_{\delta}} \mathbb{E}\left[\log \cosh \left(\xi_{\delta, x}\right)\right]=\frac{1}{2}\|\hat{\lambda}\|_{L^{2}(\Omega)}^{2} \delta^{-\frac{1}{4}}+o(1),
$$

and the pre-factor in (8.3) can be rewritten as

$$
\exp \left\{\sum_{x \in \Omega_{\delta}}\left(\log \cosh \left(\xi_{\delta, x}\right)-\mathbb{E}\left[\log \cosh \left(\xi_{\delta, x}\right)\right]\right)\right\}(1+o(1)) .
$$

The sum is over $\left|\Omega_{\delta}\right|=O\left(\delta^{-2}\right)$ i.i.d. centered random variables, hence it converges to zero in probability provided $\operatorname{Var}\left[\log \cosh \left(\xi_{\delta, x}\right)\right]=o\left(\delta^{2}\right)$. This is checked by a Taylor expansion:

$$
\operatorname{Var}\left[\log \cosh \left(\xi_{\delta, x}\right)\right] \leq \mathbb{E}\left[\left(\log \cosh \left(\xi_{\delta, x}\right)\right)^{2}\right]=O\left(\lambda_{\delta, x}^{4}\right)=O\left(\delta^{\frac{7}{2}}\right)=o\left(\delta^{2}\right)
$$

Step 2. It remains to verify that the sum in (8.3) converges to the desired Wiener chaos expansion, namely (3.37). Defining the family $\zeta_{\delta}=\left(\zeta_{\delta, x}\right)_{x \in \Omega_{\delta}}$ by

$$
\zeta_{\delta, x}:=\frac{\tanh \left(\xi_{\delta, x}\right)}{\operatorname{Var}\left(\tanh \left(\xi_{\delta, x}\right)\right)^{1 / 2}},
$$

the sum in (8.3) can be written as a polynomial chaos expansion $\Psi_{\delta}\left(\zeta_{\delta}\right):=\sum_{I \subseteq \Omega_{\delta}} \psi_{\delta}(I) \zeta_{\delta, \text {, }}^{I}$, where

$$
\psi_{\delta}(I):=\operatorname{Var}\left(\tanh \left(\xi_{\delta, \cdot}\right)\right)^{\frac{|I|}{2}} \mathrm{E}_{\Omega_{\delta}}^{+}\left[\sigma_{I}\right], \quad I \subseteq \Omega_{\delta} .
$$

We are thus left with checking that the conditions in Theorem 2.3 are satisfied.

By a Taylor expansion, as $\delta \downarrow 0$ one has

$$
\begin{aligned}
\mathbb{E}\left[\tanh \left(\xi_{\delta, x}\right)\right] & =h_{\delta, x}+O\left(h_{\delta, x}^{3}+\lambda_{\delta, x}^{3}\right)=\hat{h}(x) \delta^{\frac{15}{8}}+O\left(\delta^{\frac{21}{8}}\right), \\
\mathbb{E}\left[\tanh \left(\xi_{\delta, x}\right)^{2}\right] & =\lambda_{\delta, x}^{2}+O\left(h_{\delta, x}^{2}+\lambda_{\delta, x}^{4}\right)=\hat{\lambda}(x)^{2} \delta^{\frac{7}{4}}+O\left(\delta^{\frac{7}{2}}\right),
\end{aligned}
$$

where the $O(\cdot)$ terms are uniform in $x$, by the continuity of $\hat{\lambda}, \hat{h}$. Therefore, uniformly in $x$,

$$
\mu_{\delta}(x):=\mathbb{E}\left[\zeta_{\delta, x}\right]=\frac{\hat{h}(x)}{\hat{\lambda}(x)} \delta+o(\delta), \quad \sigma_{\delta}^{2}:=\operatorname{Var}\left[\zeta_{\delta, x}\right]=1 .
$$

Recalling that $v_{\delta}=\delta^{2}$ and $\hat{\lambda}: \bar{\Omega} \rightarrow(0, \infty)$ is continuous (hence uniformly bounded away from zero), condition (ii) of Theorem 2.3 is satisfied, with $\boldsymbol{\sigma}_{0}=1$ and $\boldsymbol{\mu}_{0}(x):=\hat{h}(x) / \hat{\lambda}(x)$.

The uniform integrability of $\left(\left(\zeta_{\delta, x}-\mu_{\delta}(x)\right)^{2}\right)_{\delta \in(0,1), x \in \Omega_{\delta}}$ holds because the moments $\mathbb{E}\left[\left(\zeta_{\delta, x}-\mu_{\delta}(x)\right)^{4}\right]$ are uniformly bounded, as for the disordered pinning model, cf. (6.7).

Step 3. It remains to check conditions (iii) and (iii) of Theorem 2.3, By (8.7)

$$
\operatorname{Var}\left[\tanh \left(\xi_{\delta, x}\right)\right]=\hat{\lambda}(x)^{2} \delta^{\frac{7}{4}}+O\left(\delta^{\frac{7}{2}}\right),
$$

hence for fixed $I=\left\{x_{1}, \ldots, x_{n}\right\} \subseteq \Omega$, by (8.6),

$$
\psi_{\delta}(I)=\hat{\lambda}(\cdot)^{|I|} \delta^{\frac{7}{8}|I|} \mathrm{E}_{\Omega_{\delta}}^{+}\left[\sigma_{.}^{I}\right]=\hat{\lambda}\left(x_{1}\right) \cdots \hat{\lambda}\left(x_{n}\right) \delta^{\frac{7}{8} n} \mathrm{E}_{\Omega_{\delta}}^{+}\left[\sigma_{x_{1}} \cdots \sigma_{x_{n}}\right] .
$$


Recalling that $v_{\delta}=\delta^{2}$, relation (3.33), that was recently proved by Chelkak, Hongler and Izyurov [CHI12, yields immediately that for every $n \in \mathbb{N}$ and distinct $x_{1}, \ldots, x_{n} \in \Omega$

$$
\lim _{\delta \downarrow 0} v_{\delta}^{-\frac{n}{2}} \psi_{\delta}\left(\left\{x_{1}, \ldots, x_{n}\right\}\right)=\hat{\lambda}\left(x_{1}\right) \cdots \hat{\lambda}\left(x_{n}\right) \mathcal{C}^{n} \phi_{\Omega}^{+}\left(x_{1}, \ldots, x_{n}\right)=: \psi_{0}\left(\left\{x_{1}, \ldots, x_{n}\right\}\right) .
$$

(Incidentally, since $\boldsymbol{\sigma}_{0}=1$ and $\boldsymbol{\mu}_{0}(x):=\hat{h}(x) / \hat{\lambda}(x)$, the Wiener chaos expansion of Theorem 2.3, cf. (2.12), matches with the one of Theorem 3.14, cf. (3.37).)

To extend the pointwise convergence (8.8) to $L^{2}$ convergence, we need uniform bounds on $\psi_{\delta}(I)$. By Lemma 8.1 below, we have the following bound uniformly in $\delta \in(0,1)$ :

$$
v_{\delta}^{-\frac{|I|}{2}} \psi_{\delta}(I) \leq\left(C\|\hat{\lambda}\|_{\infty}\right)^{|I|} \prod_{i=1}^{|I|} \frac{1}{d\left(x_{i}, \partial \Omega \cup I \backslash\left\{x_{i}\right\}\right)^{\frac{1}{8}}}=:\left(C\|\hat{\lambda}\|_{\infty}\right)^{|I|} f_{\Omega}\left(x_{1}, \ldots, x_{|I|}\right),
$$

where by Lemma 8.3 below, given $|I|=n$ for any $n \in \mathbb{N}$,

$$
\frac{1}{n !}\left\|f_{\Omega}\right\|_{L^{2}\left(\Omega^{n}\right)}^{2} \leq C^{n}(n !)^{-\frac{3}{4}} .
$$

Combined with (8.8), it follows that conditions (iii) and (iii) of Theorem 2.3 are satisfied and this completes the proof of Theorem 3.14.

We next state and prove the lemmas needed to establish (8.9) and (8.10).

Lemma 8.1. Let $\Omega, \Omega_{\delta}$ for $\delta>0$, and $\mathrm{E}_{\Omega_{\delta}}^{+}$be as introduced at the beginning of this section. Then there exists $C=C(\Omega) \in(0, \infty)$ such that for any $I=\left\{x_{1}, \ldots, x_{n}\right\} \subseteq \Omega$ with $|I|=n$

$$
0 \leq \mathrm{E}_{\Omega_{\delta}}^{+}\left[\sigma_{x_{1}} \cdots \sigma_{x_{n}}\right] \leq C^{n} \delta^{\frac{n}{8}} \prod_{i=1}^{n} \frac{1}{d\left(x_{i}, \partial \Omega \cup I \backslash\left\{x_{i}\right\}\right)^{\frac{1}{8}}},
$$

where for any $x \in \Omega$ we define $\sigma_{x}:=\sigma_{x_{\delta}}$, with $x_{\delta}$ being the point in $\Omega_{\delta}$ closest to $x$, and we set $d(x, A):=\inf _{y \in A}\|x-y\|$.

Proof. If $B(x ; r)$ denotes a ball of radius $r$ centered at $x$, and $\mathbb{B}(x ; r)_{\delta}:=B(x ; r) \cap(\delta \mathbb{Z})^{2}$, then (3.33) with $\Omega=B(0 ; 1), n=1$ and $x_{1}=0$ implies that for some $C \in(0, \infty)$

$$
\mathrm{E}_{\mathbb{B}(0 ; 1)_{\delta}}^{+}\left[\sigma_{0}\right] \leq C \delta^{\frac{1}{8}} \quad \text { for all } \delta \in(0,1) .
$$

Then, for any $x \in \Omega$, by imposing + boundary condition on the ball $B(x ; r)$ with radius $r:=d(x, \partial \Omega)$ and applying the FKG inequality [G06, Chapter 2], we obtain

$$
\mathrm{E}_{\Omega_{\delta}}^{+}\left[\sigma_{x}\right] \leq \mathrm{E}_{\mathbb{B}(x ; r)_{\delta}}^{+}\left[\sigma_{x}\right]=\mathrm{E}_{\mathbb{B}(0 ; r)_{\delta}}^{+}\left[\sigma_{0}\right]=\mathrm{E}_{\mathbb{B}(0 ; 1)_{\delta / r}}^{+}\left[\sigma_{0}\right] \leq C \frac{\delta^{\frac{1}{8}}}{r^{\frac{1}{8}}}=\frac{C \delta^{\frac{1}{8}}}{d(x, \partial \Omega)^{\frac{1}{8}}},
$$

where in the last inequality we applied (8.12).

Relation (8.11) follows by applying (8.13) and Lemma 8.2 below, choosing $\Omega_{i}$ therein to be $\Omega_{i} \cap(\delta \mathbb{Z})^{2}$, where $\Omega_{i}$ is the ball centered at $x_{i}$ with radius $\frac{1}{4} d\left(x_{i}, \partial \Omega \cup I \backslash\left\{x_{i}\right\}\right)$.

Lemma 8.2. Let $x_{1}, \ldots, x_{n} \in \Omega \subseteq \mathbb{Z}^{2}$ and suppose that $x_{i} \in \Omega_{i} \subseteq \Omega$, with $\Omega_{i} \cap\left(\Omega_{j} \cup \partial \Omega_{j}\right)=$ $\emptyset$ for all $i \neq j$. Then

$$
0 \leq \mathrm{E}_{\Omega}^{+}\left[\sigma_{x_{1}} \cdots \sigma_{x_{n}}\right] \leq \prod_{i=1}^{n} \mathrm{E}_{\Omega_{i}}^{+}\left[\sigma_{i}\right]
$$


Proof. Relation (8.14) is a consequence of the Griffiths-Kelly-Sherman (GKS) inequalities (see e.g. [E06, Chapter V.3]). We recall that $\mathrm{P}_{\Omega}^{+}$denotes the Ising measure on $\{ \pm 1\}^{\Omega}$ with inverse temperature $\beta \in(0, \infty)$ and zero external field, cf. (3.30). (The fact that $\beta=\beta_{c}$ is immaterial for this proof, and we could even include a positive external field in $\mathrm{P}_{\Omega}^{+}$.) Given $h=\left(h_{x}\right)_{x \in \Omega}$, let $\mathrm{P}_{\Omega, h}^{\text {free }}$ denote the Ising measure with external (site-dependent) field $h$, i.e.

$$
\mathrm{P}_{\Omega, h}^{\mathrm{free}}(\sigma)=\frac{1}{Z_{\Omega, \beta, h}^{\text {free }}} \exp \left\{\sum_{x \sim y \in \Omega} \beta \sigma_{x} \sigma_{y}+\sum_{x \in \Omega} h_{x} \sigma_{x}\right\} .
$$

Since $\mathrm{P}_{\Omega}^{+}=\mathrm{P}_{\Omega, h^{+}}^{\text {free }}$ with the choice $h_{x}^{+}:=\beta|\{y \in \partial \Lambda: y \sim x\}|$, we may focus on $\mathrm{P}_{\Omega, h}^{\text {free }}$.

Let $I:=\left\{x_{1}, \ldots, x_{n}\right\}$ and $\sigma_{I}:=\sigma_{x_{1}} \cdots \sigma_{x_{n}}$. If $h \geq 0$ (that is, $h_{x} \geq 0$ for all $x \in \Omega$ ),

$$
\mathrm{E}_{\Omega, h}^{\text {free }}\left[\sigma_{I}\right] \geq 0,
$$

by the first GKS inequality, proving the first bound in (8.14). Always for $h \geq 0$,

$$
\frac{\partial \mathrm{E}_{\Omega, h}^{\text {free }}\left[\sigma_{I}\right]}{\partial h_{y}}=\mathrm{E}_{\Omega, h}^{\text {free }}\left[\sigma_{I} \sigma_{y}\right]-\mathrm{E}_{\Omega, h}^{\text {free }}\left[\sigma_{I}\right] \mathrm{E}_{\Omega, h}^{\text {free }}\left[\sigma_{y}\right] \geq 0, \quad \forall y \in \Omega,
$$

by the second GKS inequality. Therefore $\mathrm{E}_{\Omega, h}^{\mathrm{free}}\left[\sigma_{I}\right]$ is increasing in $h_{y}$ for every $y \in \Omega$. Starting with $h=h^{+}$and increasing $h_{y}$ to $+\infty$ for each $y \in \cup_{i=1}^{n} \partial \Omega_{i}$, the resulting Ising measure is equivalent to imposing + boundary condition on $\cup_{i=1}^{n} \partial \Omega_{i}$. Under this limiting measure, the distribution of the spin configurations on the disjoint subdomains $\left(\Omega_{i}\right)_{1 \leq i \leq n}$ factorizes, leading to the second bound in (8.14).

Lemma 8.3. For any $n \in \mathbb{N}$ and distinct $x_{1}, \ldots, x_{n} \in \Omega$, set $I:=\left\{x_{1}, \ldots, x_{n}\right\}$ and define

$$
f_{\Omega}\left(x_{1}, \ldots, x_{n}\right):=\prod_{i=1}^{n} \frac{1}{d\left(x_{i}, \partial \Omega \cup I \backslash\left\{x_{i}\right\}\right)^{\frac{1}{8}}} .
$$

Then there exists $C=C(\Omega)<\infty$, such that for all $n \in \mathbb{N}$,

$$
\left\|f_{\Omega}\right\|_{L^{2}\left(\Omega^{n}\right)}^{2} \leq C^{n}(n !)^{\frac{1}{4}} .
$$

Proof. To prove (8.16), it suffices to show that for all $n \in \mathbb{N}$

$$
\left\|f_{\Omega}\right\|_{L^{2}\left(\Omega^{n}\right)}^{2} \leq C n^{\frac{1}{4}}\left\|f_{\Omega}\right\|_{L^{2}\left(\Omega^{n-1}\right)}^{2},
$$

where $\left\|f_{\Omega}\right\|_{L^{2}\left(\Omega^{0}\right)}^{2}:=1$.

Note that in

$$
\left\|f_{\Omega}\right\|_{L^{2}\left(\Omega^{n}\right)}^{2}=\int \cdots \int \prod_{\Omega^{n}}^{n} \frac{1}{d\left(x_{i}, \partial \Omega \cup I \backslash\left\{x_{i}\right\}\right)^{\frac{1}{4}}} \mathrm{~d} x_{1} \cdots \mathrm{d} x_{n},
$$

we can divide the domain of integration $\Omega$ for $x_{n}$ into disjoint open sets $\Omega_{0}, \ldots, \Omega_{n-1}$ (modulo a set of measure 0 ), such that

$$
\begin{aligned}
& x_{n} \in \Omega_{0} \quad \text { if and only if } \quad d\left(x_{n}, \partial \Omega \cup I \backslash\left\{x_{n}\right\}\right)=d\left(x_{n}, \partial \Omega\right), \\
& x_{n} \in \Omega_{i} \quad \text { if and only if } \quad d\left(x_{n}, \partial \Omega \cup I \backslash\left\{x_{n}\right\}\right)=d\left(x_{n}, x_{i}\right), \quad 1 \leq i \leq n-1 .
\end{aligned}
$$

We next bound $f_{\Omega}\left(x_{1}, \ldots, x_{n}\right)$ in terms of $f_{\Omega}\left(x_{1}, \ldots, x_{n-1}\right)$. First consider the case $x_{n} \in$ $\Omega_{0}$. For each $1 \leq i \leq n-1$, either

$$
d\left(x_{i}, \partial \Omega \cup I \backslash\left\{x_{i}\right\}\right)=d\left(x_{i}, \partial \Omega \cup I^{\prime} \backslash\left\{x_{i}\right\}\right),
$$


where $I^{\prime}:=\left\{x_{1}, \ldots, x_{n-1}\right\}$, or

$$
d\left(x_{i}, \partial \Omega \cup I \backslash\left\{x_{i}\right\}\right)=d\left(x_{i}, x_{n}\right) .
$$

In the later case, by the triangle inequality and the assumption $x_{n} \in \Omega_{0}$, we find that

$$
d\left(x_{i}, \partial \Omega\right) \leq d\left(x_{i}, x_{n}\right)+d\left(x_{n}, \partial \Omega\right) \leq 2 d\left(x_{i}, x_{n}\right),
$$

and hence

$$
\frac{1}{d\left(x_{i}, \partial \Omega \cup I \backslash\left\{x_{i}\right\}\right)}=\frac{1}{d\left(x_{i}, x_{n}\right)} \leq \frac{2}{d\left(x_{i}, \partial \Omega\right)} \leq \frac{2}{d\left(x_{i}, \partial \Omega \cup I^{\prime} \backslash\left\{x_{i}\right\}\right)} .
$$

If $N_{0}$ denotes the number of points among $\left\{x_{1}, \ldots, x_{n-1}\right\}$ such that (8.21) holds, then

$$
f_{\Omega}^{2}\left(x_{1}, \ldots, x_{n}\right) \leq 2^{\frac{N_{0}}{4}} f_{\Omega}^{2}\left(x_{1}, \ldots, x_{n-1}\right) \frac{1}{d\left(x_{n}, \partial \Omega\right)^{\frac{1}{4}}} .
$$

We claim that $N_{0} \leq 6$, which would then imply

$$
\int_{\Omega^{n-1} \times \Omega_{0}} \cdots \int_{\Omega} f_{\Omega}^{2}\left(x_{1}, \ldots, x_{n}\right) \mathrm{d} x_{1} \cdots \mathrm{d} x_{n} \leq 2^{\frac{3}{2}}\left\|f_{\Omega}\right\|_{L^{2}\left(\Omega^{n-1}\right)}^{2} \int_{\Omega} \frac{\mathrm{d} x_{n}}{d\left(x_{n}, \partial \Omega\right)^{\frac{1}{4}}},
$$

where the last integral is bounded by some constant $C_{3}(\Omega)<\infty$, because $\Omega$ is assumed to be a bounded simply connected domain with a piecewise smooth boundary.

To verify the claim that $N_{0} \leq 6$, assume without loss of generality that $x_{1}, \ldots, x_{k}$ are the points which satisfy (8.21). In particular, $d\left(x_{i}, x_{n}\right) \leq d\left(x_{i}, x_{j}\right)$ for all $1 \leq i \neq j \leq k$. We may shift the origin to $x_{n}$ and assume without loss of generality that $x_{i} \in \mathbb{R}^{2}$ has polar coordinates $\left(r_{i}, \vartheta_{i}\right)$, and the directional vectors $e^{i \vartheta_{1}}, \ldots, e^{i \vartheta_{k}}$ are ordered counter clockwise on the unit circle. For any two adjacent $e^{i \vartheta_{j}}$ and $e^{i \vartheta_{j+1}}$ on the unit circle, in order to satisfy

$$
\max \left\{d\left(x_{j}, 0\right), d\left(x_{j+1}, 0\right)\right\} \leq d\left(x_{j}, x_{j+1}\right),
$$

it is necessary that the angle between $e^{i \vartheta_{j}}$ and $e^{i \vartheta_{j+1}}$ is at least $\frac{\pi}{3}$. It then follows that there can be at most 6 such points.

We now consider the case $x_{n} \in \Omega_{i}$ for $1 \leq i \leq n-1$ (recall (8.19)). Without loss of generality, assume that $x_{n} \in \Omega_{1}$. By the same reasoning as above, for each $1 \leq i \leq n-1$, either relation (8.20) or relation (8.21) holds; in the latter case we can replace (8.22) by

$$
d\left(x_{i}, x_{1}\right) \leq d\left(x_{i}, x_{n}\right)+d\left(x_{n}, x_{1}\right) \leq 2 d\left(x_{i}, x_{n}\right),
$$

because $x_{n} \in \Omega_{1}$. Thus for $i \geq 2$ relation (8.23) still holds if we replace $d\left(x_{i}, \partial \Omega\right)$ by $d\left(x_{i}, x_{1}\right)$ therein. The case $i=1$ needs to be dealt with separately: for this we simply bound

$$
\begin{aligned}
\frac{1}{d\left(x_{1}, \partial \Omega \cup I \backslash\left\{x_{1}\right\}\right)^{\frac{1}{4}}} & \leq \frac{1}{d\left(x_{1}, \partial \Omega \cup I^{\prime} \backslash\left\{x_{1}\right\}\right)^{\frac{1}{4}}}+\frac{1}{d\left(x_{1}, x_{n}\right)^{\frac{1}{4}}} \\
& =\frac{1}{d\left(x_{1}, \partial \Omega \cup I^{\prime} \backslash\left\{x_{1}\right\}\right)^{\frac{1}{4}}}\left(1+\frac{d\left(x_{1}, \partial \Omega \cup I^{\prime} \backslash\left\{x_{1}\right\}\right)^{\frac{1}{4}}}{d\left(x_{1}, x_{n}\right)^{\frac{1}{4}}}\right) .
\end{aligned}
$$

We thus obtain the following analogue of (8.24) (with $N_{0} \leq 6$ ) when $x_{n} \in \Omega_{1}$ :

$$
f_{\Omega}^{2}\left(x_{1}, \ldots, x_{n}\right) \leq 2^{\frac{3}{2}} f_{\Omega}^{2}\left(x_{1}, \ldots, x_{n-1}\right)\left\{\left(1+\frac{d\left(x_{1}, \partial \Omega \cup I^{\prime} \backslash\left\{x_{1}\right\}\right)^{\frac{1}{4}}}{d\left(x_{1}, x_{n}\right)^{\frac{1}{4}}}\right) \frac{1}{d\left(x_{n}, x_{1}\right)^{\frac{1}{4}}}\right\} .
$$


Bounding the term in brackets by $C_{4}(\Omega) / d\left(x_{1}, x_{n}\right)^{\frac{1}{2}}$, for some $C_{4}(\Omega)<\infty$ (recall that $\Omega$ is a bounded set), we obtain

$$
\begin{aligned}
\int_{\Omega^{n-1} \times \Omega_{1}} \cdots \int_{\Omega} f_{\Omega}^{2}\left(x_{1}, \ldots, x_{n}\right) \mathrm{d} x_{1} \cdots \mathrm{d} x_{n} & \leq 2^{\frac{3}{2}} C_{4}(\Omega) \iint_{\Omega^{n-1} \times \Omega_{1}} \frac{f_{\Omega}^{2}\left(x_{1}, \ldots, x_{n-1}\right)}{d\left(x_{n}, x_{1}\right)^{\frac{1}{2}}} \mathrm{~d} x_{1} \cdots \mathrm{d} x_{n} \\
& \leq C_{5}(\Omega)\left|\Omega_{1}\right|^{\frac{3}{4}}\left\|f_{\Omega}\right\|_{L^{2}\left(\Omega^{n-1}\right)}^{2},
\end{aligned}
$$

for some $C_{5}(\Omega)<\infty$, where we applied the Hardy-Littlewood rearrangement inequality (see e.g. [LL01, Theorem 3.4]) to bound

$$
\int_{\Omega_{1}} \frac{\mathrm{d} x_{n}}{d\left(x_{n}, x_{1}\right)^{\frac{1}{2}}} \leq \int_{\Omega_{1}^{*}} \frac{\mathrm{d} x_{n}}{d\left(x_{n}, 0\right)^{\frac{1}{2}}}=\int_{0}^{r_{*}} \frac{2 \pi r}{r^{\frac{1}{2}}} \mathrm{~d} r=\frac{4}{3} \pi^{\frac{1}{4}}\left|\Omega_{1}\right|^{\frac{3}{4}},
$$

where $\Omega_{1}^{*}$ is the ball centered at the origin with the same area $\left|\Omega_{1}^{*}\right|=\pi r_{*}^{2}$ as $\Omega_{1}$.

Combining (8.25) and (8.26), and the analogue for $x_{n} \in \Omega_{i}$ with $2 \leq i \leq n-1$, we obtain

$$
\begin{aligned}
\left\|f_{\Omega}\right\|_{L^{2}\left(\Omega^{n}\right)}^{2} & \leq C_{6}(\Omega)\left(1+\left|\Omega_{1}\right|^{\frac{3}{4}}+\cdots+\left|\Omega_{n-1}\right|^{\frac{3}{4}}\right)\left\|f_{\Omega}\right\|_{L^{2}\left(\Omega^{n-1}\right)}^{2} \\
& \leq C_{6}(\Omega)\left(1+(n-1)\left(\frac{\left|\Omega_{1}\right|+\cdots\left|\Omega_{n-1}\right|}{n-1}\right)^{\frac{3}{4}}\right)\left\|f_{\Omega}\right\|_{L^{2}\left(\Omega^{n-1}\right)}^{2} \\
& \leq C_{2}(\Omega) n^{\frac{1}{4}}\left\|f_{\Omega}\right\|_{L^{2}\left(\Omega^{n-1}\right)}^{2},
\end{aligned}
$$

where we applied Jensen's inequality to the function $g(x)=x^{\frac{3}{4}}$. This establishes (8.17) and concludes the proof of Theorem 3.14 .

Proof of Corollary 3.16, Let $\varphi^{\prime}(\cdot)$ denote the complex derivative of the conformal map $\varphi: \widetilde{\Omega} \rightarrow \Omega$. Since $\left|\varphi^{\prime}(z)\right|^{2}$ equals the Jacobian determinant of $\varphi$, for all $f, g \in L^{2}(\Omega)$ we have

$$
\int_{\Omega} f(x) g(x) \mathrm{d} x=\int_{\widetilde{\Omega}} f(\varphi(z)) g(\varphi(z))\left|\varphi^{\prime}(z)\right|^{2} \mathrm{~d} z,
$$

by the change of variables formula. As a consequence, if $W(\cdot)$ denotes white noise on $\mathbb{R}^{2}$, the processes $\left(\int_{\Omega} f(x) W(\mathrm{~d} x)\right)_{f \in L^{2}(\Omega)}$ and $\left(\int_{\widetilde{\Omega}} f(\varphi(z))\left|\varphi^{\prime}(z)\right| W(\mathrm{~d} z)\right)_{f \in L^{2}(\Omega)}$ have the same distribution: they are both centered Gaussian processes with the same covariance (8.28). This extends to an equality in distribution for multiple integrals (recall Subsection 2.1):

$$
\int \cdots \int_{\Omega^{n}} f\left(x_{1}, \ldots, x_{n}\right) \prod_{i=1}^{n} W\left(\mathrm{~d} x_{i}\right) \stackrel{d}{=} \int \cdots \int_{\widetilde{\Omega}^{n}} f\left(\varphi\left(z_{1}\right), \ldots, \varphi\left(z_{n}\right)\right) \prod_{i=1}^{n}\left[\left|\varphi^{\prime}\left(z_{i}\right)\right| W\left(\mathrm{~d} z_{i}\right)\right],
$$

jointly for $n \in \mathbb{N}$ and symmetric $f \in L^{2}\left(\Omega^{n}\right)$. Informally, we have $W(\mathrm{~d} \varphi(z)) \stackrel{d}{=}\left|\varphi^{\prime}(z)\right| W(\mathrm{~d} z)$, which is the stochastic analogue of $\mathrm{d} \varphi(z)=\left|\varphi^{\prime}(z)\right|^{2} \mathrm{~d} z$. Recalling (3.37), it follows that

$$
\begin{array}{r}
Z_{\Omega, \hat{\lambda}, \hat{h}}^{+, W} \stackrel{d}{=} 1+\sum_{n=1}^{\infty} \frac{\mathcal{C}^{n}}{n !} \int \cdots \int_{\widetilde{\Omega}^{n}} \phi_{\Omega}^{+}\left(\varphi\left(z_{1}\right), \ldots, \varphi\left(z_{n}\right)\right) \prod_{i=1}^{n}[ \\
{\left[\hat{\lambda}\left(\varphi\left(z_{i}\right)\right)\left|\varphi^{\prime}\left(z_{i}\right)\right| W\left(\mathrm{~d} z_{i}\right)\right.} \\
\left.+\hat{h}\left(\varphi\left(z_{i}\right)\right)\left|\varphi^{\prime}\left(z_{i}\right)\right|^{2} \mathrm{~d} z_{i}\right] .
\end{array}
$$

By [CHI12, Theorem 1.3], the function $\phi_{\Omega}^{+}$is conformally covariant with

$$
\phi_{\Omega}^{+}\left(\varphi\left(z_{1}\right), \ldots, \varphi\left(z_{n}\right)\right)=\phi_{\widetilde{\Omega}}^{+}\left(z_{1}, \ldots, z_{n}\right) \prod_{i=1}^{n}\left|\varphi^{\prime}\left(z_{i}\right)\right|^{-\frac{1}{8}}
$$


hence $\boldsymbol{Z}_{\Omega, \hat{\lambda}, \hat{h}}^{+, W} \stackrel{d}{=} \boldsymbol{Z}_{\widetilde{\Omega}, \widetilde{\lambda}, \widetilde{h}}^{+, W}$, with $\widetilde{\lambda}(z):=\left|\varphi^{\prime}(z)\right|^{\frac{7}{8}} \hat{\lambda}(\varphi(z))$ and $\widetilde{h}(z):=\left|\varphi^{\prime}(z)\right|^{\frac{15}{8}} \hat{h}(\varphi(z))$.

\section{Appendix A. The Cameron-Martin shift}

Recalling Subsection 2.1 let $W=(W(f))_{f \in L^{2}\left(\mathbb{R}^{d}\right)}$ be a white noise on $\mathbb{R}^{d}$ defined on the probability space $\left(\Omega_{W}, \mathcal{A}, \mathbb{P}\right)$. We denote by $L^{0}:=L^{0}\left(\Omega_{W}, \sigma(W), \mathbb{P}\right)$ the space of (equivalence classes of) a.s. finite random variables that are measurable with respect to the $\sigma$ algebra generated by $W$, equipped with the topology of convergence in probability. Note that all the multi-dimensional stochastic integrals $W^{\otimes k}(f)$ belong to $L^{0}$.

Let us now fix $\boldsymbol{\nu} \in L^{2}\left(\mathbb{R}^{d}\right)$, representing the bias. Given $k \in \mathbb{N}$ and a symmetric squareintegrable function $f:\left(\mathbb{R}^{d}\right)^{k} \rightarrow \mathbb{R}$, the "biased stochastic integral"

$$
W_{\nu}^{\otimes k}(f)=\int \cdots \int_{\left(\mathbb{R}^{d}\right)^{k}} f\left(x_{1}, \ldots, x_{k}\right) \prod_{i=1}^{k}\left(W\left(\mathrm{~d} x_{i}\right)+\boldsymbol{\nu}\left(x_{i}\right) \mathrm{d} x_{i}\right)
$$

was defined in Remark 2.4 by expanding the product and integrating out the deterministic variables corresponding to $\boldsymbol{\nu}\left(x_{i}\right) \mathrm{d} x_{i}$, thus reducing to a sum of lower-dimensional ordinary (unbiased) stochastic integrals. In particular, for $k=1$ we can write $W_{\boldsymbol{\nu}}(f)=W_{\nu}^{\otimes 1}(f)$ as

$$
W_{\boldsymbol{\nu}}(f):=W(f)+\int_{\mathbb{R}^{d}} f(x) \boldsymbol{\nu}(x) \mathrm{d} x=W(f)+\mathbb{E}[W(f) \xi], \quad \text { with } \quad \xi:=W(\boldsymbol{\nu}),
$$

by the Itô isometry (2.2).

Thus $W_{\nu}(f)=\varrho_{\xi}(W(f))$, where we define the map $\varrho_{\xi}(X):=X+\mathbb{E}[X \xi]$ for every onedimensional stochastic integral $X$. By [J97, Theorem 14.1], such a map admits a unique extension $\varrho_{\xi}: L^{0} \rightarrow L^{0}$, called the Cameron-Martin shift, which is continuous, linear and satisfies

$$
\varrho_{\xi}(1)=1, \quad \varrho_{\xi}(X Y)=\varrho_{\xi}(X) \varrho_{\xi}(Y) \quad \forall X, Y \in L^{0} .
$$

As a consequence, the multi-dimensional biased stochastic integrals (A.1) correspond to

$$
W_{\nu}^{\otimes k}(f)=\varrho_{\xi}\left(W^{\otimes k}(f)\right) .
$$

This is easily checked for "special simple functions" $f$ (recall Subsection 2.1) using (A.2)(A.3), and is extended to general symmetric $f \in L^{2}\left(\left(\mathbb{R}^{d}\right)^{k}\right)$ using the continuity of $\varrho_{\xi}$.

For any $X \in L^{0}$, the random variable $\varrho_{\xi}(X)$ has the same distribution as $X$ under the probability $\mathbb{P}_{\boldsymbol{\nu}}$ defined in (2.15), by [J97, Theorem 14.1 (iii)-(iv)]. In particular, choosing for $X$ the series in (2.13), whenever it converges in probability, one obtains relation (2.16).

\section{Appendix B. Technical lemmas}

The following lemma is used in the proof of Theorem 2.5.

Lemma B.1 (Exponential tilting). Let $\left(\zeta_{\delta, i}\right)_{\delta \in(0,1), i \in \mathbb{T}_{\delta}}$ be a family of independent random variables in $L^{2}$, with mean $\mu_{\delta}(i)$ and variance $\sigma_{\delta}^{2}$, with $\sigma_{\delta} \rightarrow \sigma_{0} \in(0, \infty)$ and $\left\|\mu_{\delta}\right\|_{\infty}:=\sup _{i \in \mathbb{T}_{\delta}}\left|\mu_{\delta}(i)\right| \rightarrow 0$ as $\delta \downarrow 0$. Assume further that $\left(\zeta_{\delta, i}^{2}\right)_{\delta \in(0,1), i \in \mathbb{T}_{\delta}}$ are uniformly integrable. Then one can construct independent random variables $\left(\widetilde{\zeta}_{\delta, i}\right)_{\delta \in(0,1), i \in \mathbb{T}_{\delta}}$ such that

$$
\mathbb{P}\left(\widetilde{\zeta}_{\delta, i} \in \mathrm{d} x\right)=f_{\delta, i}(x) \mathbb{P}\left(\zeta_{\delta, i} \in \mathrm{d} x\right),
$$

and there exist $\delta_{0}, C \in(0, \infty)$, and $C_{p} \in(0, \infty)$ for every $p \in \mathbb{R}$, such that

$$
\mathbb{E}\left[\widetilde{\zeta}_{\delta, i}\right]=0, \quad \mathbb{E}\left[\widetilde{\zeta}_{\delta, i}^{2}\right] \leq \sigma_{\delta}^{2}\left(1+C\left|\mu_{\delta}(i)\right|\right), \quad \text { and } \quad \mathbb{E}\left[f_{\delta, i}\left(\zeta_{\delta, i}\right)^{p}\right] \leq 1+C_{p} \mu_{\delta}(i)^{2},
$$


for all $\delta \in\left(0, \delta_{0}\right)$ and $i \in \mathbb{T}_{\delta}$. Furthermore, if

$$
\inf _{\delta \in(0,1), i \in \mathbb{T}_{\delta}} \min \left\{\mathbb{P}\left(\zeta_{\delta, i}>0\right), \mathbb{P}\left(\zeta_{\delta, i}<0\right), \operatorname{Var}\left(\zeta_{\delta, i} \mid \zeta_{\delta, i}>0\right), \operatorname{Var}\left(\zeta_{\delta, i} \mid \zeta_{\delta, i}<0\right)\right\}>0,
$$

then there exists $C^{\prime} \in(0, \infty)$ such that the following improved bound holds:

$$
\mathbb{E}\left[\widetilde{\zeta}_{\delta, i}^{2}\right] \leq \sigma_{\delta}^{2}\left(1+C^{\prime} \mu_{\delta}(i)^{2}\right) .
$$

The proof of Lemma B.1 is an easy corollary of the following general result, which concerns exponential tilting of a single random variable in order to shift its mean to zero (since the random variables are not assumed to have finite exponential moments, the tilting is performed on a bounded subset). The assumptions in Lemma B.1 guarantee that conditions (B.5) and (B.9) are fulfilled, and the constants in (2.15), (B.7), (B.10) are uniformly bounded.

Theorem B.2. Let $X$ be a square-integrable random variable and let $A>0$ be such that

$$
\mathbb{E}\left[X^{2} \mathbb{1}_{\{|X|>A\}}\right] \leq \frac{1}{4} \mathbb{E}\left[X^{2}\right] .
$$

Assume that $\mathbb{E}[X]$ is sufficiently small, more precisely,

$$
|\mathbb{E}(X)| \leq \varepsilon:=\frac{\mathbb{E}\left[X^{2}\right]^{2}}{144 A^{3}}
$$

Then one can define a random variable $\tilde{X}$, such that $\mathbb{P}(\widetilde{X} \in \mathrm{d} x)=f(x) \mathbb{P}(X \in \mathrm{d} x)$, satisfying

$$
\begin{array}{r}
\mathbb{E}\left[f(X)^{p}\right] \leq 1+C_{p} \mathbb{E}[X]^{2} \quad \forall p \in \mathbb{R}, \quad \text { with } C_{p}:=\frac{4 e^{|p|}}{A \varepsilon}, \\
\mathbb{E}[\widetilde{X}]=0, \quad \mathbb{E}\left[\widetilde{X}^{2}\right] \leq \mathbb{E}\left[X^{2}\right]+C|\mathbb{E}[X]|, \quad \text { with } C:=\frac{A^{3 / 2}}{\sqrt{\varepsilon}} .
\end{array}
$$

If $\mathbb{E}[X] \geq 0$, and $A$ is chosen such that

$$
\mathbb{E}\left[X^{2} \mathbb{1}_{\{X>A\}}\right] \leq \frac{1}{4} \mathbb{E}\left[X^{2} \mathbb{1}_{\{X \geq 0\}}\right],
$$

(replace $X$ by $-X$ if $\mathbb{E}[X] \leq 0)$, and further assume that

$$
|\mathbb{E}[X \mid X \geq 0]| \leq \varepsilon^{\prime}:=\frac{\mathbb{E}\left[X^{2} \mid X \geq 0\right]^{2}}{144 A^{3}},
$$

then we can define $\tilde{X}$ such that (B.6) holds with $\varepsilon$ replaced by $\varepsilon^{\prime}$, while (B.7) is improved to

$$
\mathbb{E}[\tilde{X}]=0, \quad \mathbb{E}\left[\tilde{X}^{2}\right] \leq \mathbb{E}\left[X^{2}\right]+C^{\prime} \mathbb{E}[X]^{2}, \quad \text { with } \quad C^{\prime}:=\frac{A}{2 \mathbb{P}(X \geq 0) \varepsilon^{\prime}}
$$

Proof. Without loss of generality, we assume that $\mathrm{E}[X] \geq 0$ (otherwise consider $-X$ ).

Step 1 (Strategy). We will fix $I \subseteq \mathbb{R}$, which is either $[-A, A]$ (assuming (B.4)-(B.5)), or $[0, A]$ (assuming (B.8)-(B.9) $)$, and we define random variables $Y, Z$ with laws

$$
\mathbb{P}(Y \in \cdot):=\mathbb{P}(X \in \cdot \mid X \in I), \quad \mathbb{P}(Z \in \cdot):=\mathbb{P}(X \in \cdot \mid X \notin I) .
$$

Taking independent copies of $X, Y, Z$, we have the following equality in distribution:

$$
X \stackrel{\text { dist }}{=} \mathbb{1}_{\{X \in I\}} Y+\mathbb{1}_{\{X \notin I\}} Z, \quad \text { (note that }|Y| \leq A \text { ). }
$$


We then exponentially tilt $Y$, defining for $\lambda \in \mathbb{R}$ a random variable $Y_{\lambda}$ with law

$$
\mathbb{P}\left(Y_{\lambda} \in \mathrm{d} x\right):=e^{\lambda x-F(\lambda)} \mathbb{P}(Y \in \mathrm{d} x), \quad \text { where } \quad F(\lambda):=\log \mathbb{E}\left[e^{\lambda Y}\right] .
$$

As we will show at the end of the proof, we can choose $\lambda=\widetilde{\lambda} \in \mathbb{R}$ such that

$$
\mathbb{E}\left[Y_{\widetilde{\lambda}}\right]=-\frac{\mathbb{P}(X \notin I)}{\mathbb{P}(X \in I)} \mathbb{E}[Z]
$$

If we define $\widetilde{X}$ replacing $Y$ by $Y_{\widetilde{\lambda}}$ in the definition (B.12), that is

$$
\widetilde{X}:=\mathbb{1}_{\{X \in I\}} Y_{\widetilde{\lambda}}+\mathbb{1}_{\{X \notin I\}} Z,
$$

then $\mathbb{E}[\tilde{X}]=0$ by construction. Moreover $\mathbb{P}(\tilde{X} \in \mathrm{d} x)=f(x) \mathbb{P}(X \in \mathrm{d} x)$ with density

$$
f(x)=e^{\widetilde{\lambda} x-F(\widetilde{\lambda})} \mathbb{1}_{\{x \in I\}}+\mathbb{1}_{\{x \in \mathbb{R} \backslash I\}}=1+\left(e^{\widetilde{\lambda} x-F(\widetilde{\lambda})}-1\right) \mathbb{1}_{\{x \in I\}} .
$$

The rest of the proof is devoted to estimating $\mathbb{E}\left[\widetilde{X}^{2}\right]$ and $\mathbb{E}\left[f(X)^{p}\right]$. We are going to use the following bounds on $\widetilde{\lambda}$, which will be proved at the very end:

$$
\begin{aligned}
& I:=[-A, A], \text { assuming }(\underline{\text { B.4 }})-(\overline{B .5}): \quad|\widetilde{\lambda}| \leq \frac{|\mathbb{E}[X]|}{2 A^{3 / 2} \sqrt{\varepsilon}} ; \\
& I:=[0, A], \text { assuming }(\underline{\mathrm{B} .8})-(\overline{\mathrm{B} .9}): \quad \quad|\widetilde{\lambda}| \leq \frac{|\mathbb{E}[X]|}{\sqrt{6} A^{3 / 2} \sqrt{\varepsilon^{\prime}} \sqrt{\mathbb{P}(X \in I) \mathbb{P}(X \geq 0)}} ; \\
& \text { In either case: } \quad|\widetilde{\lambda}| \leq \frac{1}{27 A} .
\end{aligned}
$$

Step 2 (Bounds on $\mathbb{E}\left[\tilde{X}^{2}\right]$ ). Denote $G(\lambda):=\mathbb{E}\left[Y_{\lambda}^{2}\right]=\mathbb{E}\left[Y^{2} e^{\lambda Y}\right] / \mathbb{E}\left[e^{\lambda Y}\right]$. Recalling (B.15) and (B.12), we can write

$$
\mathbb{E}\left[\widetilde{X}^{2}\right]=\mathbb{E}\left[X^{2}\right]+\mathbb{P}(X \in I)\left(\mathbb{E}\left[Y_{\tilde{\lambda}}^{2}\right]-\mathbb{E}\left[Y^{2}\right]\right)=\mathbb{E}\left[X^{2}\right]+\mathbb{P}(X \in I) \int_{0}^{\widetilde{\lambda}} G^{\prime}(\lambda) \mathrm{d} \lambda .
$$

Since $G^{\prime}(\lambda)=\mathbb{E}\left[\left(Y_{\lambda}\right)^{3}\right]-\mathbb{E}\left[\left(Y_{\lambda}\right)^{2}\right] \mathbb{E}\left[Y_{\lambda}\right]$ and $\left|Y_{\lambda}\right| \leq A$, we have $\left|G^{\prime}(\lambda)\right| \leq 2 A^{3}$, and hence

$$
\mathbb{E}\left[\widetilde{X}^{2}\right] \leq \mathbb{E}\left[X^{2}\right]+2 A^{3} \mathbb{P}(X \in I)|\widetilde{\lambda}| .
$$

Applying (B.17), we obtain precisely the second bound in (B.7).

To prove (B.10), let us assume (B.8)-(B.9) and set $I:=[0, A]$. By (B.12) $(\underline{B} .14)$, we have

$$
\mathbb{E}\left[Y_{\widetilde{\lambda}}\right]=\mathbb{E}[Y]-\frac{\mathbb{E}[X]}{\mathbb{P}(X \in I)},
$$

and hence $\mathbb{E}\left[Y_{\widetilde{\lambda}}\right] \leq \mathbb{E}[Y]$. Since $\mathbb{E}\left[Y_{\lambda}\right]=F^{\prime}(\lambda)$ is increasing in $\lambda$ (because $F^{\prime \prime}(\lambda)=\operatorname{Var}\left[Y_{\lambda}\right] \geq$ 0 ), it follows that $\tilde{\lambda} \leq 0$. We then refine (B.20) as follows:

$$
\mathbb{E}\left[\widetilde{X}^{2}\right]=\mathbb{E}\left[X^{2}\right]+\mathbb{P}(X \in I) \widetilde{\lambda} G^{\prime}(0)+\mathbb{P}(X \in I) \int_{0}^{\widetilde{\lambda}}\left(\int_{0}^{\lambda} G^{\prime \prime}(s) \mathrm{d} s\right) \mathrm{d} \lambda .
$$

Note that $G^{\prime}(0)=\mathbb{E}\left[Y^{3}\right]-\mathbb{E}\left[Y^{2}\right] \mathbb{E}[Y] \geq 0$, because $Y \in[0, A]$ and hence $Y^{2}$ and $Y$ are positively correlated. Therefore the second term in (B.23) is bounded by 0 . Also note that

$$
G^{\prime \prime}(\lambda)=\mathbb{E}\left[\left(Y_{\lambda}\right)^{4}\right]-2 \mathbb{E}\left[\left(Y_{\lambda}\right)^{3}\right] \mathbb{E}\left[Y_{\lambda}\right]+2 \mathbb{E}\left[\left(Y_{\lambda}\right)^{2}\right] \mathbb{E}\left[Y_{\lambda}\right]^{2}-\mathbb{E}\left[\left(Y_{\lambda}\right)^{2}\right]^{2},
$$

and hence $\left|G^{\prime \prime}(\lambda)\right| \leq 6 A^{4}$. Substituting into (B.23) then yields

$$
\mathbb{E}\left[\widetilde{X}^{2}\right] \leq \mathbb{E}\left[X^{2}\right]+3 A^{4} \mathbb{P}(X \in I) \widetilde{\lambda}^{2} .
$$

Applying (B.18), we obtain precisely (B.10). 
Step 3 (Bounds on $\mathbb{E}\left[f(X)^{p}\right]$ ). Recall $f$ from $(\overline{\mathrm{B} .16})$ and $F(\lambda)$ from (B.13). Since $F(0)=0$ and $\left|F^{\prime}(\lambda)\right|=\left|\mathbb{E}\left[Y_{\lambda}\right]\right| \leq A$, cf. ( $(\overline{B .13})$, we have $|F(\widetilde{\lambda})| \leq A|\widetilde{\lambda}|$ and hence $|\widetilde{\lambda} x-F(\widetilde{\lambda})| \leq 2 A|\widetilde{\lambda}|$ for every $x \in I \subseteq[-A, A]$. Applying (B.19), we obtain

$$
e^{-2 / 27} \leq f(x) \leq e^{2 / 27}, \quad \forall x \in \mathbb{R} .
$$

For any $p \in \mathbb{R}$, Taylor expansion gives $y^{p} \leq 1+p(y-1)+C_{p}^{\prime}(y-1)^{2}$ for all $y \in\left[e^{-2 / 27}, e^{2 / 27}\right]$, with

$$
C_{p}^{\prime}:=\max _{y \in\left[e^{-2 / 27}, e^{2 / 27}\right]}\left|p(p-1) y^{p-2}\right|=|p(p-1)| e^{\frac{2}{27}|p-2|} \leq 2 e^{|p|} .
$$

Therefore

$$
\begin{aligned}
f(x)^{p} & \leq 1+p(f(x)-1)+C_{p}^{\prime}(f(x)-1)^{2} \\
& =1+\left(p-2 C_{p}^{\prime}\right)(f(x)-1)+C_{p}^{\prime}\left(e^{2 \widetilde{\lambda} x-2 F(\widetilde{\lambda})}-1\right) \mathbb{1}_{\{x \in I\}} .
\end{aligned}
$$

Since $f$ is a probability density, recalling the definition of $F$ from (B.13), we obtain

$$
\mathbb{E}\left[f(X)^{p}\right] \leq 1+C_{p}^{\prime} \mathbb{P}(X \in I)\left(e^{F(2 \widetilde{\lambda})-2 F(\widetilde{\lambda})}-1\right) .
$$

Since $\left|F^{\prime \prime}(\lambda)\right|=\left|\mathbb{E}\left[Y_{\lambda}^{2}\right]-\mathbb{E}\left[Y_{\lambda}\right]^{2}\right| \leq 2 A^{2}$, the Mean Value Theorem and (B.19) yield

$$
0 \leq F(2 \widetilde{\lambda})-2 F(\widetilde{\lambda})=(F(2 \widetilde{\lambda})-F(\widetilde{\lambda}))-(F(\widetilde{\lambda})-F(0)) \leq 4 A^{2} \widetilde{\lambda}^{2} \leq \frac{4}{27^{2}} \leq 1,
$$

where the first inequality holds by convexity of $F$ (note that $F^{\prime \prime}(\lambda)=\operatorname{Var}\left[Y_{\lambda}\right] \geq 0$ ).

Consider first the case $I=[-A, A]$, assuming (B.4)-(B.5) : since $e^{x}-1 \leq 2 x$ for $0 \leq x \leq 1$, applying (B.28), (B.17) and (B.26) we obtain

$$
\mathbb{E}\left[f(X)^{p}\right] \leq 1+2 C_{p}^{\prime}(F(2 \widetilde{\lambda})-2 F(\widetilde{\lambda})) \leq 1+C_{p}^{\prime} 8 A^{2} \widetilde{\lambda}^{2} \leq 1+\frac{4 e^{|p|}}{A \varepsilon} \mathbb{E}[X]^{2},
$$

proving (B.6).

The case $I=[0, A]$, assuming (B.8)-(B.9), is similar: we keep the term $\mathbb{P}(X \in I)$ in (B.27) when writing (B.29), so that applying (B.18) gives

$$
\mathbb{E}\left[f(X)^{p}\right] \leq 1+C_{p}^{\prime} \mathbb{P}(X \in I) 8 A^{2} \widetilde{\lambda}^{2} \leq 1+\frac{16 e^{|p|}}{6 A \varepsilon^{\prime}} \mathbb{E}[X]^{2} \leq 1+\frac{4 e^{|p|}}{A \varepsilon^{\prime}} \mathbb{E}[X]^{2},
$$

which coincides with (B.6), where $\varepsilon$ is replaced by $\varepsilon^{\prime}$.

Step 4 (Bounds on $\tilde{\lambda}$ ). We finally show that one can choose $\lambda=\widetilde{\lambda}$ so that (B.14) holds and the bounds (B.17)-(B.18)-(B.19) are satisfied.

Since $F^{\prime}(\lambda)=\mathbb{E}\left[Y_{\lambda}\right]$, cf. (B.13), we can rewrite (B.14), cf. (B.22), as

$$
F^{\prime}(\widetilde{\lambda})-F^{\prime}(0)=\widetilde{x}, \quad \text { where } \quad \widetilde{x}:=-\frac{\mathbb{E}[X]}{\mathbb{P}(X \in I)} .
$$

Since $F^{\prime \prime \prime}(\lambda)=\mathbb{E}\left[Y_{\lambda}^{3}\right]-3 \mathbb{E}\left[Y_{\lambda}\right] \mathbb{E}\left[Y_{\lambda}^{2}\right]+2 \mathbb{E}\left[Y_{\lambda}\right]^{3}$ and $\left|Y_{\lambda}\right| \leq A$, we have $\left|F^{\prime \prime \prime}(\lambda)\right| \leq 6 A^{3}$. Therefore

$$
F^{\prime \prime}(\lambda) \geq F^{\prime \prime}(0)-6 A^{3}|\lambda| \geq \frac{F^{\prime \prime}(0)}{2} \quad \text { for all } \quad|\lambda| \leq c:=\frac{F^{\prime \prime}(0)}{12 A^{3}}=\frac{\operatorname{Var}(Y)}{12 A^{3}} .
$$

In particular, equation (B.30) has exactly one solution $\widetilde{\lambda} \in[-c, c]$, provided

$$
|\widetilde{x}| \leq \frac{F^{\prime \prime}(0)}{2} c, \quad \text { that is } \quad|\mathbb{E}[X]| \leq \frac{\mathbb{P}(X \in I) \operatorname{Var}(Y)^{2}}{24 A^{3}},
$$


in which case $\widetilde{\lambda}$ satisfies

$$
|\widetilde{\lambda}| \leq \frac{|\widetilde{x}|}{\frac{1}{2} F^{\prime \prime}(0)}=\frac{2|\mathbb{E}[X]|}{\mathbb{P}(X \in I) \operatorname{Var}(Y)} .
$$

It only remains to check that condition (B.31) is indeed satisfied, under either assumptions (B.4)-(B.5) or (B.8)-(B.9), and to show that (B.32) yields the bounds (B.17)-(B.18) $-(\bar{B} .19)$. For this we need to estimate $\mathbb{P}(X \in I)$ and $\operatorname{Var}(Y)$.

In order to avoid repetitions, let $\mathbb{P}^{*}$ denote the original law $\mathbb{P}$ when we assume (B.4)-(B.5) or the conditional law $\mathbb{P}(\cdot \mid X \geq 0)$ when we assume (B.8)-(B.9). In either case $I=[-A, A]$ or $I=[0, A]$ we can write $\mathbb{P}(Y \in \cdot):=\mathbb{P}(X \in \cdot \mid X \in I)=\mathbb{P}^{*}(X \in \cdot|| X \mid \leq A)$, therefore

$$
\operatorname{Var}(Y)=\operatorname{Var}^{*}(X|| X \mid \leq A) .
$$

Note that assumptions (B.4)-(B.5) and (B.8)-(B.9) can be written as follows:

$$
\mathbb{E}^{*}\left[X^{2} \mathbb{1}_{\{|X|>A\}}\right] \leq \frac{1}{4} \mathbb{E}^{*}\left[X^{2}\right], \quad\left|\mathbb{E}^{*}(X)\right| \leq \frac{\mathbb{E}^{*}\left[X^{2}\right]^{2}}{144 A^{3}}
$$

Since $\mathbb{E}^{*}\left[X^{2} \mathbb{1}_{\{|X| \leq A\}}\right] \leq A^{2} \mathbb{P}^{*}(|X| \leq A)$, it follows that

$$
A^{2} \geq \frac{3}{4} \frac{\mathbb{E}^{*}\left[X^{2}\right]}{\mathbb{P}^{*}(|X| \leq A)} \geq \frac{3}{4} \mathbb{E}^{*}\left[X^{2}\right]
$$

We thus get

$$
\begin{gathered}
\mathbb{P}^{*}(|X| \leq A)=1-\mathbb{P}^{*}(|X|>A) \geq 1-\frac{\mathbb{E}^{*}\left[X^{2} \mathbb{1}_{\{|X|>A\}}\right]}{A^{2}} \geq 1-\frac{\mathbb{E}^{*}\left[X^{2}\right]}{4 A^{2}} \geq \frac{2}{3}, \\
\left|\mathbb{E}^{*}\left[X \mathbb{1}_{\{|X|>A\}}\right]\right| \leq \frac{\mathbb{E}^{*}\left[X^{2} \mathbb{1}_{\{|X|>A\}}\right]}{A} \leq \frac{\mathbb{E}^{*}\left[X^{2}\right]}{4 A} \leq \frac{\sqrt{\mathbb{E}^{*}\left[X^{2}\right]}}{2 \sqrt{3}}
\end{gathered}
$$

Together with (B.34) and (B.35), this gives

$$
\left|\mathbb{E}^{*}\left[X \mathbb{1}_{\{|X| \leq A\}}\right]\right| \leq\left|\mathbb{E}^{*}[X]\right|+\left|\mathbb{E}^{*}\left[X \mathbb{1}_{\{|X|>A\}}\right]\right| \leq\left(\frac{\left(\frac{4}{3}\right)^{3 / 2}}{144}+\frac{1}{2 \sqrt{3}}\right) \sqrt{\mathbb{E}^{*}\left[X^{2}\right]} \leq \frac{1}{3} \sqrt{\mathbb{E}^{*}\left[X^{2}\right]}
$$

which yields

$$
\left|\mathbb{E}^{*}[X|| X \mid \leq A]\right|=\frac{\left|\mathbb{E}^{*}\left[X \mathbb{1}_{\{|X| \leq A\}}\right]\right|}{\mathbb{P}^{*}(|X| \leq A)} \leq \frac{1}{2} \sqrt{\mathbb{E}^{*}\left[X^{2}\right]}
$$

Applying one more time (B.34) we get

$$
\mathbb{E}^{*}\left[X^{2}|| X \mid \leq A\right]=\frac{\left|\mathbb{E}^{*}\left[X^{2} \mathbb{1}_{\{|X| \leq A\}}\right]\right|}{\mathbb{P}^{*}(|X| \leq A)} \geq \frac{3}{4} \mathbb{E}^{*}\left[X^{2}\right],
$$

which finally yields, cf. (B.33),

$$
\operatorname{Var}(Y)=\mathbb{E}^{*}\left[X^{2}|| X \mid \leq A\right]-\mathbb{E}^{*}[X|| X \mid \leq A]^{2} \geq \frac{1}{2} \mathbb{E}^{*}\left[X^{2}\right]
$$

By (B.5) and (B.9), and applying (B.37) and (B.36), we obtain

$$
\left|\mathbb{E}^{*}[X]\right| \leq \frac{\mathbb{E}^{*}\left[X^{2}\right]^{2}}{144 A^{3}} \leq \frac{\operatorname{Var}(Y)^{2}}{36 A^{3}} \leq \frac{\mathbb{P}^{*}(|X| \leq A) \operatorname{Var}(Y)^{2}}{24 A^{3}}
$$

Consider first the case $I=[-A, A]$, assuming (B.4)-(B.5) : since $\mathbb{P}^{*}(|X| \leq A)=\mathbb{P}(X \in I)$, relation (B.38) concides precisely with the condition (B.31) to be checked. Next we consider the case $I=[0, A]$, assuming $(\overline{\mathrm{B} .8})-(\overline{\mathrm{B} .9})$, where we recall that $\mathbb{P}^{*}(\cdot)=\mathbb{P}(\cdot \mid X \geq 0)$. By 
assumption $\mathbb{E}[X] \geq 0$, we have $|\mathbb{E}[X]| \leq\left|\mathbb{E}\left[X \mathbb{1}_{\{X \geq 0\}}\right]\right|=\mathbb{P}(X \geq 0)\left|\mathbb{E}^{*}[X]\right|$. Since we can write $\mathbb{P}^{*}(|X| \leq A)=\mathbb{P}(X \in I) / \mathbb{P}(X \geq 0)$, relation (B.38) again yields (B.31).

To conclude, for the case $I=[-A, A]$, applying (B.36) and (B.37) to (B.32) and recalling the definition of $\varepsilon$ in (B.5) gives (B.17). For the case $I=[0, A]$, the bound (B.18) follows similarly, recalling the definition of $\varepsilon^{\prime}$ in (B.9) and observing that $\mathbb{P}(X \in I) \geq \frac{2}{3} \mathbb{P}(X \geq 0)$ by (B.36). Finally, to obtain (B.19) from (B.17) $($ (B.18), apply (B.35) and the assumptions (B.4), (B.8).

Finally, we prove the following bound on iterated integrals.

Lemma B.3. Let $\chi \in[0,1)$. Then there exist $c_{1}, c_{2}>0$ such that for all $k \in \mathbb{N}$,

$$
\int_{0<t_{1}<\cdots<t_{k}<1} \cdots \int_{1} \frac{\mathrm{d} t_{1} \cdots \mathrm{d} t_{k}}{t_{1}^{\chi} \cdots\left(t_{k}-t_{k-1}\right) \chi} \leq c_{1} e^{-c_{2} k \log k},
$$

and

$$
\int_{0<t_{1}<\cdots<t_{k}<1} \cdots \int_{1} \frac{\mathrm{d} t_{1} \cdots \mathrm{d} t_{k}}{t_{1}^{\chi} \cdots\left(t_{k}-t_{k-1}\right)^{\chi}\left(1-t_{k}\right)^{\chi}} \leq c_{1} e^{-c_{2} k \log k} .
$$

Proof. It is enough to prove (B.40), since the integral therein bounds (B.39). Recognizing the density of the Dirichlet distribution (with parameters $k+1$ and $1-\chi$ ) allows to evaluate

$$
\int_{0<t_{1}<\cdots<t_{k}<1} \cdots \int_{1} \frac{\mathrm{d} t_{1} \cdots \mathrm{d} t_{k}}{t_{1}^{\chi} \cdots\left(t_{k}-t_{k-1}\right)^{\chi}\left(1-t_{k}\right)^{\chi}}=\frac{\Gamma(1-\chi)^{k+1}}{\Gamma((k+1)(1-\chi))},
$$

and (B.40) follows by standard properties of the gamma function $\Gamma(\cdot)$.

\section{ACKNOWLEDGEMENTS}

We are grateful to Tom Spencer for fruitful discussions and we thank the referee for helpful comments. We also thank Niccolò Torri and Ran Wei for spotting some mistakes in the draft. We acknowledge support of NUS grant R-146-000-148-112 (R.S.), a Marie Curie International Reintegration Grant within the 7th European Community Framework Programme IRG-246809 and EPSRC grant EP/L012154/1 (N.Z.), and ERC Advanced Grant 267356 VARIS (F.C.). We also thank the kind hospitality of the Institute of Mathematics at the University of Leiden, of Academia Sinica in Taipei and of the Department of Mathematics at Hokkaido University in Sapporo, where parts of this work were completed.

\section{REFERENCES}

[AW90] M. Aizenman and J. Wehr. Rounding effects of quenched randomness on first-order phase transitions. Commun. Math. Phys. 130, 489-528, 1990.

[A08] K. S. Alexander. The effect of disorder on polymer depinning transitions. Commun. Math. Phys. 279 (2008), 117-146.

[AZ09] K. S. Alexander and N. Zygouras. Quenched and annealed critical points in polymer pinning models. Commun. Math. Phys. 291 (2009), 659-689.

[AKQ14a] T. Alberts, K. Khanin, and J. Quastel. The intermediate disorder regime for directed polymers in dimension $1+1$. Ann. Probab. 42 (2014), 1212-1256.

[AKQ14b] T. Alberts, K. Khanin, and J. Quastel. The Continuum Directed Random Polymer. J. Stat. Phys. 154 (2014), 305-326.

[ACQ11] G. Amir, I. Corwin, and J. Quastel. Probability distribution of the free energy of the continuum directed random polymer in 1+1 dimensions. Commun. Pure Appl. Math. 64 (2011), 466-537. 
[BCPSZ14] Q. Berger, F. Caravenna, J. Poisat, R. Sun, and N. Zygouras. The Critical Curve of the Random Pinning and Copolymer Models at Weak Coupling. Commun. Math. Phys. 326 (2014), 507530 .

[BGT87] N.H. Bingham, C.M. Goldie, J.L. Teugels. Regular variation, Encyclopedia of Mathematics and its Applications, 27. Cambridge University Press, Cambridge (1987).

[BdH97] E. Bolthausen, F. den Hollander. Localization transition for a polymer near an interface. Ann. Probab. 25 (1997), 1334-1366.

[B06] A. Bovier. Statistical mechanics of disordered systems: a mathematical perspective. Cambridge University Press, 2006.

[BKKKL92] J. Bourgain, J. Kahn, G. Kalai, Y. Katznelson, and N. Linial. The influence of variables in product spaces. Israel J. Math. 77 (1992), 55-64.

[BK88] J. Bricmont and A. Kupiainen. Phase transition in the 3d random field Ising model. Commun. Math. Phys. 116 (1988), 539-572.

[CGN12b] F. Camia, C. Garban, and C.M. Newman. The Ising magnetization exponent on $\mathbb{Z}^{2}$ is $1 / 15$. Probab. Theory Relat. Fields 160 (2014), 175-187.

[CGN12] F. Camia, C. Garban, and C.M. Newman. Planar Ising magnetization field I. Uniqueness of the critical scaling limit. Ann. Probab. 43 (2015), 528-571.

[CGN13] F. Camia, C. Garban, and C.M. Newman. Planar Ising magnetization field II. Properties of the critical and near-critical scaling limits. Ann. Inst. H. Poincaré Probab. Statist. 52 (2016), $146-161$.

[Lig68] T.L. Liggett. An invariance principle for conditioned sums of independent random variables. J. Math. Mech. 18 (1968), 559-570.

[CG10] F. Caravenna, G. Giacomin. The weak coupling limit of disordered copolymer models. Ann. Probab. 38 (2010), 2322-2378.

[CSZ14] F. Caravenna, R. Sun, N. Zygouras. The continuum disordered pinning model. Probab. Theory Relat. Fields 164 (2016), 17-59.

[Car88] J. Cardy. Finite size scaling. North Holland Pub. Co. (1988).

[C06] S. Chatterjee. A generalization of the Lindeberg principle. Ann. Probab. 34 (2006), 2061-2076.

[CD13] S. Chatterjee, P. S. Dey. Multiple phase transitions in long-range first-passage percolation on square lattices. Commun. Pure Appl. Math. 69 (2016), 203-256.

[CCFS86] J.T. Chayes, L. Chayes, D.S. Fischer, and T. Spencer. Finite-Size Scaling and Correlation Lengths for Disordered Systems. Phys. Rev. Lett. 57, number 24, 15 December 1986.

[CCFS89] J.T. Chayes, L. Chayes, D.S. Fischer, and T. Spencer. Correlation Length Bounds for Disordered Ising Ferromagnets. Commun. Math. Phys. 120 (1989), 501-523.

[CHI12] D. Chelkak, C. Hongler, and C. Izyurov. Conformal invariance of spin correlations in the planar Ising model. Ann. Math. 181 (2015), 1087-1138.

[CJKS14] D. Conus, M. Joseph, D. Khoshnevisan, and S-Y. Shiu. Initial measures for the stochastic heat equation. Ann. Inst. H. Poincaré Probab. Statist. 50 (2014), 136-153.

[CSY04] F. Comets, T. Shiga, and N. Yoshida. Probabilistic analysis of directed polymers in a random environment: a review. Stochastic analysis on large scale interacting systems. Adv. Stud. Pure Math. 39 (2004), 115-142. Math. Soc. Japan.

[C12] I. Corwin. The Kardar-Parisi-Zhang equation and universality class. Random Matrices Theory Appl. 1 (2012) 76 p.

[DZ] P. Dey and N. Zygouras. High temperature limits of directed polymers with heavy tail disorder. Ann. Probab. (to appear).

[D97] R.A. Doney. One-sided Local Large Deviation and Renewal Theorems in the Case of Infinite Mean. Probab. Theory Relat. Fields 107, 451-465, 1997.

[E06] R. Ellis. Entropy, large deviations, and statistical mechanics. Springer-Verlag, Berlin, 2006.

[G07] G. Giacomin. Random Polymer Models. Imperial College Press, London, 2007.

[G10] G. Giacomin. Disorder and critical phenomena through basic probability models. École d'Été de Probabilités de Saint-Flour XL - 2010. Springer Lecture Notes in Mathematics 2025.

[G06] G. Grimmett, The Random-Cluster Model. Vol. 333 of Grundlehren der Mathematischen Wissenschaften, Springer-Verlag, Berlin, 2006.

[H74] A.B. Harris, Effect of random defects on the critical behaviour of Ising models. Journal of Physics C: Solid State Physics, 1974. 
[HH85] D. A. Huse and C. L. Henley. Pinning and roughening of domain wall in Ising systems due to random impurities. Phys. Rev. Lett. 54 (1985) 270-2711.

[J97] S. Janson. Gaussian Hilbert spaces. Cambridge Tracts in Mathematics, Vol. 129. Cambridge University Press, Cambridge (1997).

[KKL88] J. Kahn, G. Kalai, and N. Linial. The influence of variables on boolean functions. Proceedings of the 29th Annual Symposium on Foundations of Computer Science (1988), 68-80.

[L10] H. Lacoin. New bounds for the free energy of directed polymer in dimension $1+1$ and $1+2$. Commun. Math. Phys. 294 (2010) 471-503.

[LL01] E.H. Lieb and M. Loss. Analysis. Second edition. Graduate Studies in Mathematics, 14. American Mathematical Society, Providence, RI, 2001.

[MOO10] E. Mossel, R. O'Donnell, and K. Oleszkiewicz. Noise stability of functions with low influences: Invariance and optimality. Ann. Math. 171 (2010), 295-341.

[PT10] G. Peccati and M.S. Taqqu. Wiener Chaos: Moments, Cumulants and Diagrams. SpringerVerlag (2010).

[SS10] T. Sasamoto and H. Spohn. Exact height distributions for the KPZ equation with narrow wedge initial condition. Nucl. Phys. B 834 (2010), 523-542.

[Soh09] J. Sohier. Finite size scaling for homogeneous pinning models. Alea 6 (2009), 163-177.

[TV11] T. Tao and V. Vu. Random matrices: Universality of the local eigenvalue statistics. Acta Mathematica 206 (2011), 127-204.

Dipartimento di Matematica e Applicazioni, Università degli Studi di Milano-Bicocca, via Cozzi 55, 20125 Milano, Italy

E-mail address: francesco.caravenna@unimib.it

Department of Mathematics, National University of Singapore, 10 Lower Kent Ridge Road, 119076 Singapore

E-mail address: matsr@nus.edu.sg

Department of Statistics, University of Warwick, Coventry CV4 7AL, UK

E-mail address: N.Zygouras@warwick.ac.uk 